\title{
Free-Space Optical Beam Steering for Wireless Communications
}

\author{
by \\ Christopher Mekhiel \\ Bachelor of Applied Science, University of Waterloo, 2015 \\ A thesis \\ presented to Ryerson University \\ in partial fulfillment of the \\ requirements for the degree of \\ Master of Applied Science \\ in the Program of \\ Electrical and Computer Engineering
}

Toronto, Ontario, Canada, 2017

(C)Christopher Mekhiel 2017 


\section{AUTHOR'S DECLARATION FOR ELECTRONIC SUBMISSION OF A THESIS}

I hereby declare that I am the sole author of this thesis. This is a true copy of the thesis, including any required final revisions, as accepted by my examiners.

I authorize Ryerson University to lend this thesis to other institutions or individuals for the purpose of scholarly research.

I further authorize Ryerson University to reproduce this thesis by photocopying or by other means, in total or in part, at the request of other institutions or individuals for the purpose of scholarly research.

I understand that my thesis may be made electronically available to the public. 
Free-Space Optical Beam Steering for Wireless Communications

Master of Applied Science 2017

Christopher Mekhiel

Electrical and Computer Engineering

Ryerson University

\begin{abstract}
The implementation of optical wireless communications (OWC) requires the use of a light-emittingdiode (LED) or laser diode (LD). Due to significant path loss exhibited by these sources in an outdoor environment, an unobstructed point-to-point link must be maintained in order to increase the signal-tonoise ratio (SNR) at the receiver.

This thesis considers a solution to alleviate the fundamental limitations of the OWC channel in an outdoor environment by investigating optical beam steering (OBS) to increase the signal strength in the desired direction. Conventional methods to implement an OBS use a microelectromechanical (MEMS) mirror or a spatial light modulator (SLM) which both suffer from low switching frequency. A high frequency OBS device can be created by using an optical phased array (OPA). An electro-optic modulator (EOM) such as $\mathrm{LiNbO}_{3}$ can be used to create an OPA but can not be directly integrated in silicon. For monolithic silicon-on-insulator (SOI) solutions, previous literature uses thermo-optic couplers on SOI to implement the OPA, however this introduces the issue of thermal cross-talk. Therefore, this thesis focuses on the use of silicon as the EOM for use in an OPA to create a high frequency monolithic OBS.

Our contributions consist of providing a design method for a OBS SOI device which exhibits minimum internal cross-talk and provides propagation in free-space with high directivity and a wide steering range. Additionally, we propose the use of an internal heterodyne optical phase locked loop (PLL) to stabilize the OBS instead of an external signal processor for phase correction. This optical PLL reduces beam
\end{abstract}


jitter, minimizes beam squint, and provides active tracking for the output beam towards the receiver. We have also characterized a shadowing scenario in an OWC channel which OBS has the potential to alleviate. Moreover, we simulated the optical far-field radiation pattern from a SOI waveguide to free-space which has not been demonstrated in previous research. Finally, our simulation results of a SOI OPA demonstrates the coherent combining capability of OBS using MEEPTM and the Optiwave ${ }^{\mathrm{TM}}$ suite. 


\section{Acknowledgements}

I would like to acknowledge the guidance from my thesis advisor, Professor X. Fernando at Ryerson University. Professor X. Fernando was always willing to meet me to promptly answer any questions of mine and guide me in the right direction. 


\section{Dedication}

I dedicate this to my father who was a great support for me through the years to make this possible. I also thank my sister and mother for always being there for me. 


\section{Contents}

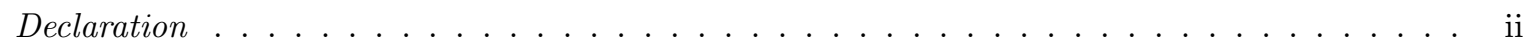

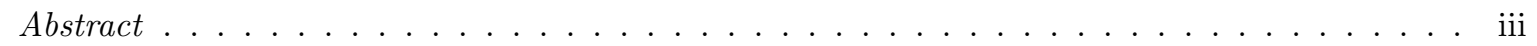

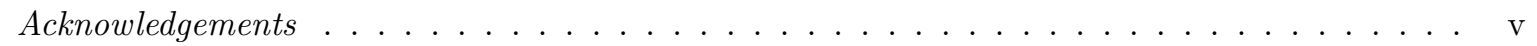

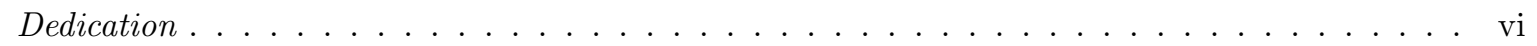

List of Tables . . . . . . . . . . . . . . . . . . . . . ix

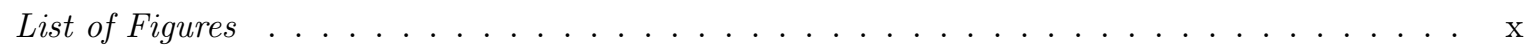

List of Acronyms . . . . . . . . . . . . . . . . . . . . . . . . xii

List of Symbols . . . . . . . . . . . . . . . . . . . . . . . . xiii

$\begin{array}{lll}1 & \text { Introduction } & 1\end{array}$

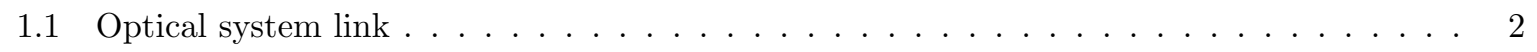

1.2 Problem statement $\ldots \ldots \ldots \ldots \ldots \ldots \ldots \ldots$

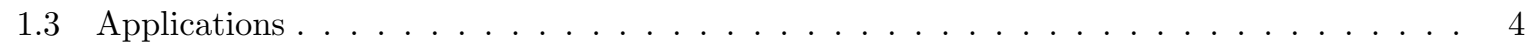

$1.3 .1 \quad$ Space-division multiple access $\ldots \ldots \ldots \ldots \ldots \ldots \ldots$. . . . . . . . . . 4

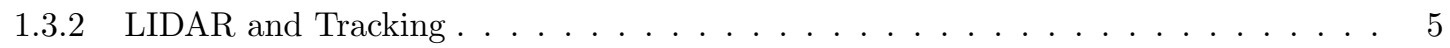

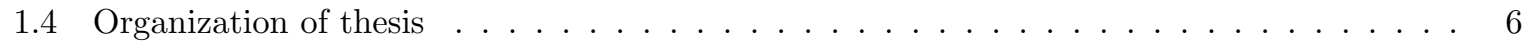

\begin{tabular}{|lll}
\hline 2 & Literature Review & \\
\hline
\end{tabular}

2.1 Optical channel characteristics $\ldots \ldots \ldots \ldots \ldots \ldots \ldots$

2.2 Techniques for OBS $\ldots \ldots \ldots \ldots \ldots \ldots \ldots \ldots \ldots$

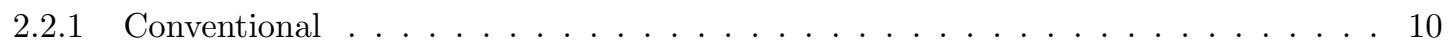

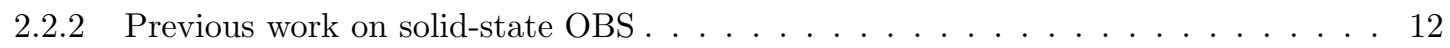

$2.2 .3 \quad$ Silicon phase modulation $\ldots \ldots \ldots \ldots \ldots \ldots \ldots$

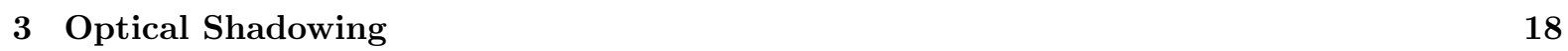

3.1 Channel modelling with shadowing $\ldots \ldots \ldots \ldots \ldots \ldots \ldots$

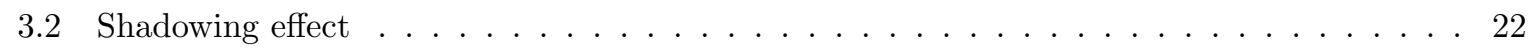

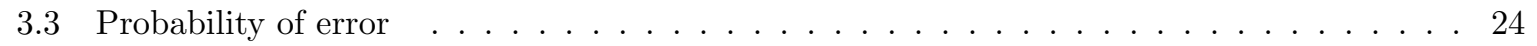

3.4 Shadowing simulations $\ldots \ldots \ldots \ldots \ldots \ldots$

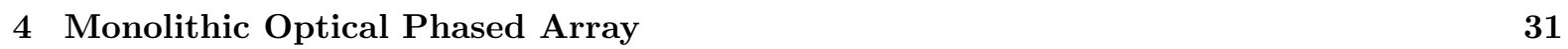

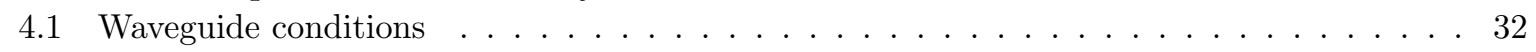

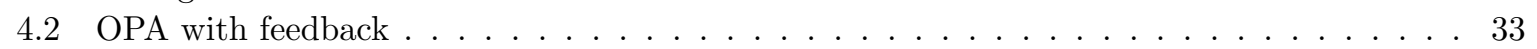

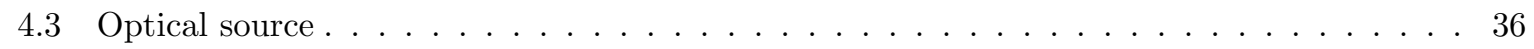

4.3 .1 LED vs Laser coherence $\ldots \ldots \ldots \ldots$. . . . . . . . . . . . . . . . . . . . . . . . . . . . . .

4.3 .2 LED to waveguide interface . . . . . . . . . . . . . . . . 36

4.4 OPA link loss estimation $\ldots \ldots \ldots \ldots \ldots \ldots$ 
\begin{tabular}{|lll}
5 & Optical Beam Steering Simulations & 43
\end{tabular}

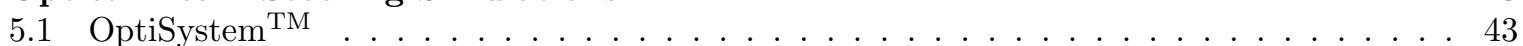

5.2 Single waveguide . . . . . . . . . . . . . . . . . . . . . . . . . . . 45

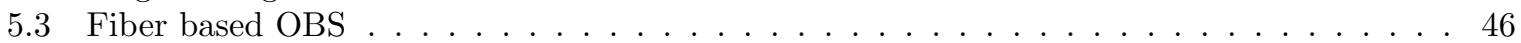

5.4 SOI OBS with OPA $\ldots \ldots \ldots \ldots \ldots \ldots$

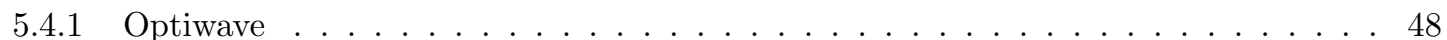

$5.4 .2 \quad$ Free-space OBS simulations $\ldots \ldots \ldots \ldots \ldots \ldots$

$5.4 .3 \quad$ HPBW and Directivity $\ldots \ldots \ldots \ldots \ldots \ldots \ldots$

\begin{tabular}{lll}
\hline 6 & Discussions and Conclusions & 57
\end{tabular}

\begin{tabular}{|l|l}
\hline Appendices & $\mathbf{5 9}$
\end{tabular}

Appendix A MEEPTM Source Code . . . . . . . . . . . . . . . . . . . . 59

A.1 CW source . . . . . . . . . . . . . . . . . . . . . . . . . . . 59

A.2 Gaussian source . . . . . . . . . . . . . . . . . . . . . . . . 59

A.3 Fiber implementation . . . . . . . . . . . . . . . . . . . . . . . 60

Appendix B Python Source Code . . . . . . . . . . . . . . . . . . . . . . . . 61

B.1 Integrated SOI . . . . . . . . . . . . . . . . . . . . . . . . 61

B.2 Fiber implementation . . . . . . . . . . . . . . . . . . . . . . . . . . 63

B.3 SOI with coupling branches $\ldots \ldots \ldots \ldots \ldots$. . . . . . . . . . . . 64

\begin{tabular}{ll}
\hline Bibliography & 71
\end{tabular} 


\section{List of Tables}

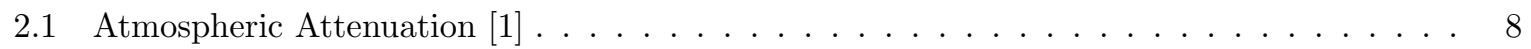

2.2 Experimental system parameters $\ldots \ldots \ldots \ldots \ldots \ldots$

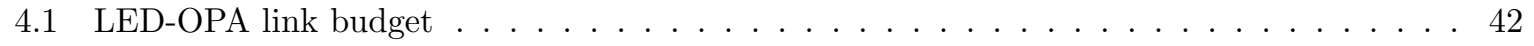

5.1 Optical beam forming radiation with an element spacing of $0.62 \mu m \ldots \ldots \ldots$

$5.2 \quad$ Optical beam forming radiation with an element spacing of $0.78 \mu \mathrm{m} \ldots \ldots$. . . . . . 55

5.3 Optical beam forming radiation with an element spacing of $0.93 \mu \mathrm{m} \ldots \ldots$. . . . . . . 56

5.4 Optical beam forming radiation with an element spacing of $1.25 \mu \mathrm{m} \ldots \ldots$ 


\section{List of Figures}

1.1 OBS SDMA . . . . . . . . . . . . . . . . . . . . . . . . . . . . . . . . 4

1.2 State-space model . . . . . . . . . . . . . . . . . . . . . . . . 5

2.1 MEMS tip-tilt mirror [2] . . . . . . . . . . . . . . . . . . . . . . . . . 11

2.2 SLM functionality $[2]$. . . . . . . . . . . . . . . . . . . . . . . . . . . . . . . . . . . 12

2.3 OPA developed by MIT 3$]$. . . . . . . . . . . . . . . . . . . . . . . . . . . . . 14

2.4 Thermal OPA developed by UCSB [4] . . . . . . . . . . . . . . . . . . . . . . . . . . . . . 15

2.5 Integrated SOI phase modulator $[5]$. . . . . . . . . . . . . . . . . . . . . . . . 16

3.1 VLC system setup . . . . . . . . . . . . . . . . . . . . . . . . . . . . 21

3.2 LED shadowing example . . . . . . . . . . . . . . . . . . . . . . . . . . . . . . . 22

3.3 LED-mild shadowing . . . . . . . . . . . . . . . . . . . . . . . . . . . . . . . 27

3.4 LED-moderate shadowing . . . . . . . . . . . . . . . . . . . . . . . . . . . . . . . 28

3.5 LED-severe shadowing . . . . . . . . . . . . . . . . . . . . . . . . . . . . . . . 28

3.6 Error probability with different shadowing effects . . . . . . . . . . . . . . . . . . . . . . 30

3.7 Multimode scenario . . . . . . . . . . . . . . . . . . . . . . . . 30

4.1 Planar waveguide geometrical constraints . . . . . . . . . . . . . . . . . . . . . . . 33

4.2 Internal OPA feedback . . . . . . . . . . . . . . . . . . . . . . . . . . . . . . . . . 34

4.3 LED butt-coupling to SMF . . . . . . . . . . . . . . . . . . . . . . . . . . 37

4.4 Butt-coupling from fibre to optical circuit . . . . . . . . . . . . . . . . . . . . . . . . . . 38

4.5 Optical ring resonator . . . . . . . . . . . . . . . . . . . . . . . . . . 39

4.6 Overall SOI OPA for LED source . . . . . . . . . . . . . . . . . . . . . . . . . . . . . . . 40

4.7 Fiber Bragg grating filter with circulator . . . . . . . . . . . . . . . . . . . . . . . . . . . . 41

$5.1 \quad$ OptiSystem $^{\mathrm{TM}}$ schematic with integrated OptiBPM ${ }^{\mathrm{TM}}$ waveguide. . . . . . . . . . . . . . 44

$5.2 \quad$ OptiSystem $^{\mathrm{TM}}$ spectrum analyzer of output . . . . . . . . . . . . . . . . . . . . . . 44

$5.3 \quad$ Polar and EM plot of single element in the far-field . . . . . . . . . . . . . . . . . . . . . . 45

5.4 Far-field radiation of fiber array implementation . . . . . . . . . . . . . . . . . . . . . . 4 47

5.5 A lens based FSO terminal . . . . . . . . . . . . . . . . . . . . . . . . . . . 47

$5.6 \quad 0.95 \mu m$ element spacing with $\beta_{1}=55^{\circ}$ and $\beta_{2}=74^{\circ}$ configuration . . . . . . . . . . . . . 48

5.7 OptiBPM ${ }^{\mathrm{TM}}$ scattering matrix simulation . . . . . . . . . . . . . . . . . . . . . . . . . 49

$5.8 \quad 0.62 \mu m$ element spacing with $\beta_{1}=55^{\circ}$ and $\beta_{2}=74^{\circ}$ configuration . . . . . . . . . . . . . . 50

5.9 Frequency variation of $0.62 \mu \mathrm{m}$ and $0.78 \mu \mathrm{m}$ element spacing with $\beta_{1}=55^{\circ}$ and $\beta_{2}=74^{\circ}$

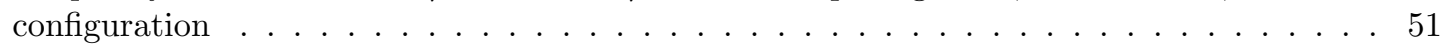

5.10 Frequency variation of $0.93 \mu \mathrm{m}$ and $1.25 \mu \mathrm{m}$ element spacing with $\beta_{1}=55^{\circ}$ and $\beta_{2}=74^{\circ}$

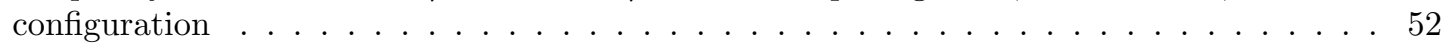

5.11 Beam angle variation for $0.62 \mu m$ and $0.78 \mu m$ element spacing . . . . . . . . . . . . . . . 52

5.12 Beam angle variation for $0.93 \mu m$ and $1.25 \mu m$ element spacing . . . . . . . . . . . . . . . 53 
$5.13 \mathrm{HPBW}$ variation with increase in element spacing $\ldots \ldots \ldots \ldots$. . . . . . . . . 53

5.14 Directivity variation with increase in element spacing . . . . . . . . . . . . . . . 54

5.15 Far-field radiation of feedback configuration $\ldots \ldots \ldots \ldots \ldots$ 


\section{List of Acronyms}

ACO-OFDM Asymmetrically Clipped Optical Orthogonal Frequency-Division Multiplexing APD Avalanche Photodiode

AWGN Additive White Gaussian noise

BER Bit-Error-Rate

CW Continuous Wave

DCO-OFDM DC biased Optical Orthogonal Frequency-Division Multiplexing

EMI Electromagnetic Interference

EOM Electro-optic Modulator

FSO Free-Space Optical

FDTD Finite-Difference-Time-Domain

FOV Field-of-View

FWHM Full width at Half Maximum

HPBW Half-Power Beam Width

IM Intensity Modulator

ISI Inter-Symbol Interference

JFTS Joint Fading and Two-path Shadowing

LD Laser Diode

LO Local Oscillator

LED Light Emitting Diode

LOS Line-of-Sight

Li-Fi Light-Fidelity

MEMS Microelectromechanical

MIMO Multiple-Input Multiple-Output

MMF Multi-Mode Fiber

MZM Mach-Zehnder Modulator

OWC Optical Wireless Communications

OOK On-Off Keying

OPA Optical Phased Array

PAM Pulse Amplitude Modulation

PD Photodiode

PDF Probability Density Function

PWM Pulse-Width Modulation

PPM Pulse-Position Modulation

PLL Phase Locked Loop

RF Radio Frequency

SDMA Space-Division Multiple Access

SNR Signal-to-Noise Ratio

SMF Single Mode Fiber

SLM Spatial Light Modulator

SCOWA Semiconductor Amplifier

SOI Silicon-On-Insulator

TTD True-Time-Delay

VLC Visible Light Communication

WDM Wavelength-Division Multiplexing 


\section{List of Symbols}

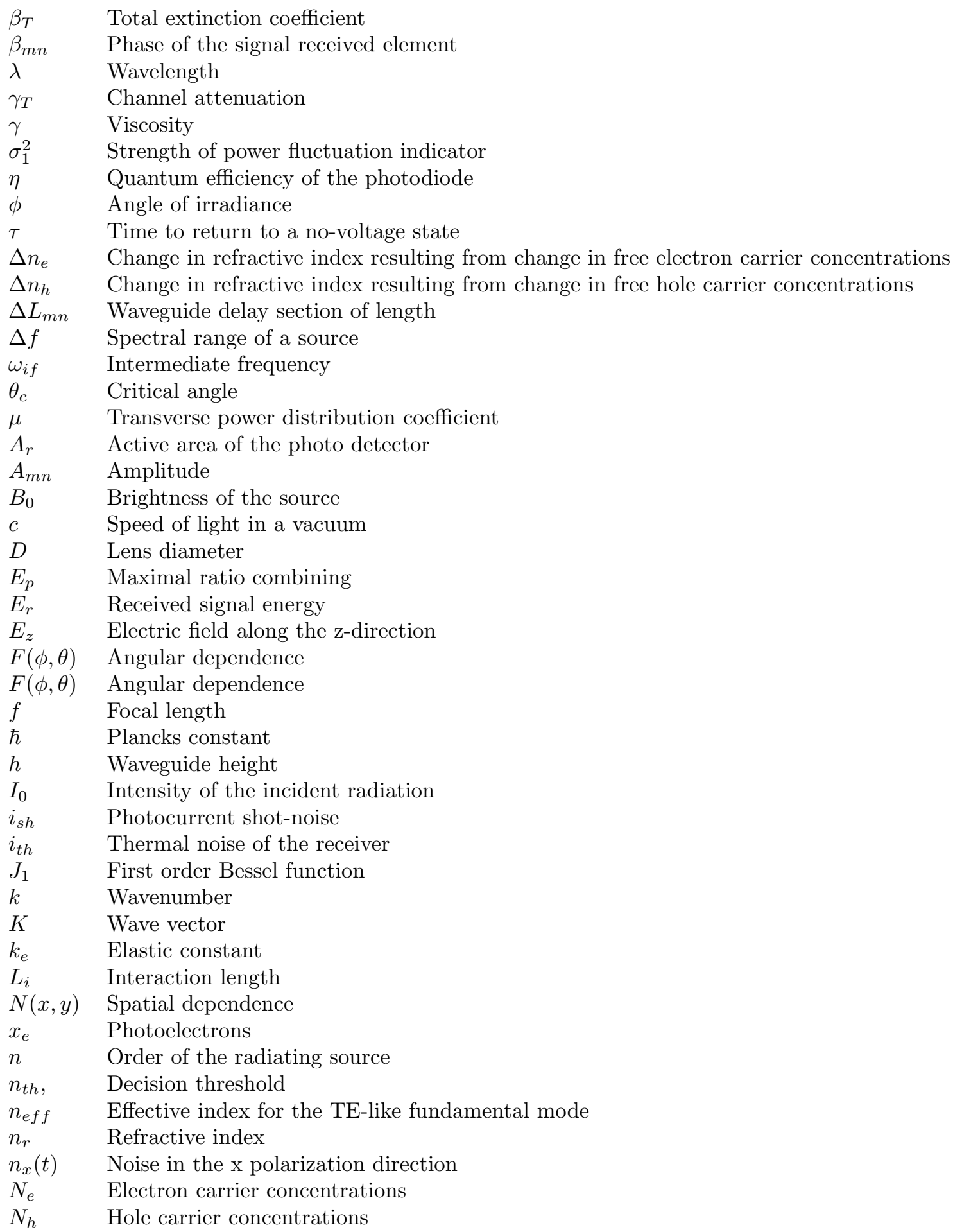


$P_{0} \quad$ Received average power

$P_{e} \quad$ Probability of Error

$R \quad$ Responsivity of the photodiode

$R_{d} \quad$ Distance between the lighting source and the surface of the detector

$r \quad$ Radial distance

$r_{0} \quad$ Represents the mode field radius of the SMF

$r_{33} \quad$ Electro-optic coefficient

$S(t) \quad$ Distance between the means of the two normal functions

$S_{m n}$ Position vector

$T_{d} \quad$ Drop transmission

$T_{p} \quad$ Pass transmission

$t \quad$ Cell thickness

$v \quad$ Lateral power distribution coefficient 


\section{Chapter 1}

\section{Introduction}

Radio frequency (RF) wireless connectivity has been used for several decades as it allows for indoor and outdoor links to be established without any physical connection. RF wireless links require that spectrum licensing fees are paid to federal regulatory bodies and are required to be contained within strict spectral zones. These frequency allocations are determined by local authorities and may vary from country to country, making a standard interface difficult. The spectral efficiency of radio transmission is saturating, hence the focus shift to alternative communication technologies. Higher frequencies such as mm-waves and above are being explored.

The broadcast nature of the RF link is beneficial for mobile connectivity but this may result in interference between devices located within close proximity. However, optical radiation in the infrared or visible range is easily contained by opaque boundaries. As a result, interference between adjacent devices can be minimized. Additionally, inexpensive light-emitting diodes (LEDs) and photodiodes (PDs) are able to interchangeably work between baseband and transmission frequencies where as high-frequency circuit design techniques are required in the RF domain. Free-space optical (FSO) transceivers use a highly directional and cone-shaped beam with a dominant line-of-sight (LOS) propagation path. Therefore, interception is extraordinarily difficult and anyone tapping into the systems can easily be detected as the intercept equipment must be placed within the 
very narrow optical foot print [6].

Optical communication research has attracted the interest of scholars around the world and has evolved into a new IEEE standard named the P802.15.7 - Standard for Short-Range Wireless Optical Communication [7]. Moreover, the Light Fidelity (Li-Fi) standard has been developed [8]. Li-Fi is a bidirectional, high-speed wireless communication technology and can also be a form of visible light communication (VLC). LiFi has the potential to be around 100 times faster than some Wi-Fi implementations, reaching speeds of $224 \mathrm{~Gb} / \mathrm{s}$ [9]. Optical wireless communications (OWC) can take advantage of LEDs or Laser Diodes (LD). Utilizing solid state LEDs for lighting purposes is energy efficient and can also be implemented for data communication. LEDs can be easily and effectively modulated by switching the light in frequencies beyond what the human eye can perceive. Hence, data can be transmitted in addition to its main lighting functionality. Conventional modulation techniques such as pulse-width modulation (PWM), pulse-position modulation (PPM), on-off keying (OOK), and pulse-amplitude modulation (PAM) are not bandwidth efficient schemes and can suffer from inter-symbol interference (ISI). Advanced (coherent) modulation schemes are not directly applicable to LEDs as their output is incoherent. However, various modulation techniques such as a DC biased optical orthogonal frequency-division multiplexing (DCO-OFDM), asymmetrically clipped optical (ACO-OFDM), etc. have been developed which are applicable to LED/LD systems.

\section{$1.1 \quad$ Optical system link}

Without any ambient noise or distortion, the transmitted bit stream should reach the destination without any errors. However, due to the internal and external noise sources, some binary data will be falsely detected which causes bit errors. In addition to the typical additive white Gaussian noise (AWGN) that is locally generated by the receiver electronic circuit, $\mathrm{PD}$ receivers are prone to the ambient radiation that can cause errors in the detection process [10]. Another factor which severely deteriorates an optical link 
is the effect of scattering in certain areas or opaque objects which may interfere within the LOS of the receiver.

A ubiquitous method to create the LOS condition in RF is known as beam forming. Beam forming is a phased array technique which directs the radiation towards a designated receiver. This effectively increases the signal-to-noise ratio (SNR) and reduces the bit-error rate (BER). In this thesis, we extend the theory of beam forming to the optical medium by means of an optical phased array (OPA) to create optical beam steering (OBS).

\subsection{Problem statement}

The main limitation of OWC is its significant path loss in an outdoor environment which greatly restricts the mobility of devices. Furthermore, the application for OWC is severely limited due to its inability to transmit through opaque boundaries. This creates a unique shadowing effect which hinders the performance of the OWC system. In order to further promote the shift to OWC, this thesis presents the use of OBS in order to increase the SNR to overcome the fundamental limitations imposed on the OWC channel.

With regards to OBS, many conventional solutions consider the use of a microelectromechanical (MEMS) mirror or a spatial light modulator (SLM) to steer the output beam. However, both solutions exhibit low switching frequency which reduce the steering speed. Additionally, most monolithic OPAs suffer from internal cross-talk and do not have the ability to stabilize the direction of the output beam. Any previous research which has demonstrated a method to stabilize the output beam did so by means of a low frequency signal processor as a feedback which limits the overall steering speed. To implement a high frequency OBS device, we propose the use of a monolithic SOI OPA which exhibits minimum internal cross talk, high directivity, and a wide steering range. To deal with the issue of stabilizing the direction of the output beam, we propose the use of an optical phased locked loop (PLL) which does not require an external signal processor to minimize beam squint, beam jitter, and provide tracking towards the receiver. 
This has the potential to enable an OPA in the OBS device to achieve phase correction on the order of $\mathrm{GHz}$.

We use OptiBPM ${ }^{\mathrm{TM}}$ and MEEP ${ }^{\mathrm{TM}}$ to design the OBS device. OptiBPM ${ }^{\mathrm{TM}}$ is a CAD environment used to design optical waveguides. MEEPTM is an open-source scheme based FDTD simulator developed by MIT 11. OptiBPM ${ }^{\mathrm{TM}}$ is used to determine the spacing and dimensions of the OPA to ensure single mode propagation throughout each waveguide branch and no internal cross coupling. Once this has been verified, MEEPTM will be used to sweep the OPA dimensions around the results from OptiBPM ${ }^{\mathrm{TM}}$ for interfacing with free-space to simulate the OBS functionality.

\subsection{Applications}

\subsubsection{Space-division multiple access}

Space-division multiple access (SDMA) can be enabled through the use of OBS. OBS provides multiple users access to the communication channel depending on their individual spatial locations. Essentially, SDMA separates users on the shared optical band. SDMA requires high frequency OBS in order to maintain simultaneous connectivity between all users as shown in Figure 1.1 .

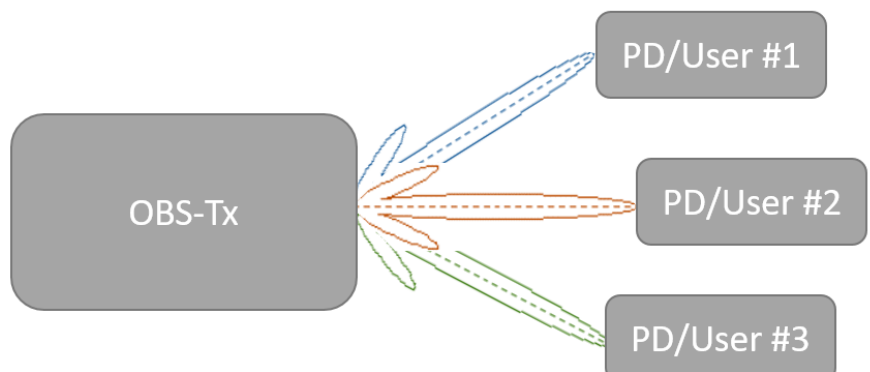

Figure 1.1: OBS SDMA 


\subsubsection{LIDAR and Tracking}

Light detection and ranging (LIDAR) [12] is an optical remote sensing technology that measures properties of scattered and reflected light to find range and other information about a target by means of OBS. LIDAR as opposed to radar uses optical frequencies and is advantageous for various applications due to it's inability to penetrate through materials. This requires the use of relatively high speed scanning to detect reflections which can be used to determine the objects speed and distance. Mechanical techniques are ubiquitous for use in LIDAR in order to steer the beam to measure reflections. Hence, a monolithic OBS which uses an OPA is an excellent replacement for mechanical LIDAR due to its high frequency steering.

In a FSO link, fine pointing and tracking towards the PD receiver is necessary to ensure that a high SNR is achieved and the BER is low. Various techniques for tracking can be implemented. For instance, state-space models are a common method with respect to high speed tracking. The EOM voltage will be the inputs for control of the OPA $(u)$, the output would be the PD matrix $(z)$, and the state variables $(x)$ are correlated to channel dynamics which may include shadowing as illustrated in Figure 1.2. The fundamental matrix can be solved for object tracking.

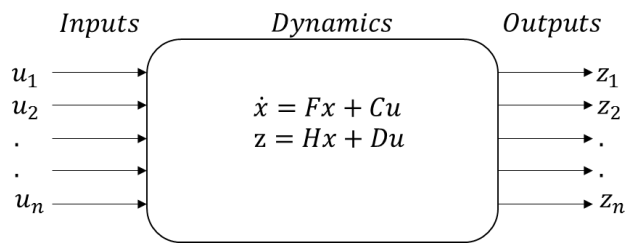

Figure 1.2: State-space model

Moreover, for short distance OWC, a reflective film can be placed around the receiver array in order for the transmitter to acknowledge the target location based on the reflected component. The method for tracking can be implemented by combining a periodic LIDAR stage followed by a tracking stage. 


\subsection{Organization of thesis}

In Chapter 2, we present a literature review on a OWC system where absorption, scattering, and polarization effects are taken into consideration along with previous OBS techniques. Chapter 2 demonstrates that if the photons received are low, then it can be characterized by a Poisson distribution. With a large photoelectron count, the probability distribution can be approximated as a Gaussian distribution. Following this approximation, in Chapter 3 we have characterized a shadowing scenario in an OWC channel by applying the Gaussian distribution to a bimodal case. It is essential to study the OWC channel effects in Chapter 2 and Chapter 3 in order to design a monolithic OBS which suffices the power requirements imposed by the OWC channel. Additionally, after characterizing the OWC channel under shadowing, we propose that the only solution to increase the SNR is by implementing OBS. The effect of scattering is not taken into consideration in the overall design of the OWC system as it would disrupt the coherence of the OBS.

Moreover, in Chapter 4, we present the design steps required to design a monolithic, solid-state OBS device. Chapter 5 illustrates our far-field simulations for the designed waveguide array which illustrates high directivity and minimum internal cross-coupling. Chapter 6 provides the discussion and conclusion of our work as well as our future work. 


\section{Chapter 2}

\section{Literature Review}

\subsection{Optical channel characteristics}

In order to design a OWC system to meet the SNR requirements with an OBS terminal, the performance of an optical system in an outdoor environment is essential to investigate. In general, attenuation is defined as the process whereby some or all of the energy of an electromagnetic (EM) wave is lost, this can include absorption or scattering effects while propagating through free space. In this thesis, since we are mainly concerned with OBS for outdoor applications, we will present the channel characteristics for atmospheric conditions.

In clear atmospheric conditions, the attenuation is typically $0.2 \mathrm{~dB} / \mathrm{km}$ and $10 \mathrm{~dB} / \mathrm{km}$ in urban areas. Absorption occurs when the energy of a photon gets converted to internal energy, caused by EM radiation impinging on matter. The absorption peak occurs at a frequency where the most power is absorbed. Hence, atmospheric absorption is wavelength dependent. The effect of scattering is also another factor which affects the transmitted beam. Scattering causes dispersal of a beam of particles or radiation into a range of directions due to physical interactions. The effect of scattering depends on the particle the EM wave interacts with (size with respect to the photon's wavelength). The main types of scattering that occur in the atmosphere are Rayleigh, Mie, and non- 
selective. Rayleigh scattering occurs when the particles are small compared to the EM wavelength. This scattering is mainly observed in gases, transparent solids or liquids. Mie scattering occurs when EM waves are incident on particles with diameters that are larger than a tenth of the incident light wavelength. Non-selective scattering occurs in particles whose size is larger than the incident wavelength. With respect to OWC, Rayleigh and Mie scattering are the most common variety of scattering. In order to calculate the attenuation that occurs from atmospheric particles, the effects of scattering and absorption must be taken into account for evaluating the extinction coefficient.

The power per unit area at a distance, $d$ along the radiation path can be calculated using the extinction coefficient as 1

$$
E_{p}=\frac{I_{0}^{-\beta_{T} d}}{d^{2}}
$$

where $E_{p}$ is the emergent power, $I_{0}$ is the intensity of the incident radiation, and $\beta_{T}$ is the total extinction coefficient. This describes the exponential attenuation of light with increasing distance. The attenuation in foggy scenarios can reach up to $300 \mathrm{~dB} / \mathrm{km}$. Table 2.1 summarizes the attenuation at different wavelengths and various visibilities. It is important to note that the infrared band undergoes less attenuation than at higher frequency bands.

Table 2.1: Atmospheric Attenuation 1

\begin{tabular}{lcc}
\hline Visibility S $(\mathrm{km})$ & $\lambda=800 n m(\mathrm{~dB} / \mathrm{km})$ & $\lambda=1500 \mathrm{~nm}(\mathrm{~dB} / \mathrm{km})$ \\
\hline 0.5 & 32.5 & 30.8 \\
0.7 & 23 & 21 \\
0.9 & 18 & 16 \\
1.1 & 14.5 & 12.5 \\
1.3 & 12 & 10 \\
1.5 & 10 & 8.33 \\
\hline
\end{tabular}

The attenuation can be characterised and modelled as [1]

$$
a=\frac{17}{d}\left(\frac{555}{\lambda}\right)^{0.195 d}
$$


Additionally, scintillation affects the optical transmission by varying the strength of the optical signal as it propagates. Scintillation reflects variations in the index of refraction of the atmosphere. The intensity fluctuations of an optical beam can be expressed as a log-normal or gamma-gamma probability density function (PDF). In general, scintillation becomes prominent over $500 \mathrm{~m}$ with a receiver which has a small aperture. The use of adaptive optics can mitigate issues caused by scintillation and scattering.

If the average received power is low, the optical atmospheric channel can be characterised by a Poisson distribution where the average received power is given as [6]

$$
P_{r}=P_{t} \exp \left(-\gamma_{T} L\right)
$$

where $\gamma_{T}$ represents the channel attenuation with the average received photoelectron count provided as [6]

$$
<x_{e}>=\frac{\eta \lambda T_{b} P_{r}}{\hbar c}
$$

where $\hbar$ and $c$ are the Plancks constant and the speed of light in a vacuum, respectively, and $\eta$ is the quantum efficiency of the PD. Moreover, the probability of receiving $x_{e}$ photoelectrons is [6]

$$
p_{1}\left(x_{e}\right)=\int_{0}^{\infty} \frac{\left(\eta \lambda T_{b} P_{r} / \hbar c\right)^{x} \exp \left(-\eta \lambda T_{b} P_{r} / \hbar c\right)}{x_{e} ! \sqrt{2 \pi \sigma_{1}^{2}} P_{r}} \exp \left[-\frac{1}{2 \sigma_{1}^{2}}\left(\ln \frac{P_{r}}{P_{0}}+\frac{\sigma_{1}^{2}}{2}\right)^{2}\right] d P_{r}
$$

where $P_{0}$ is the received average power in the absence of atmospheric turbulence and $\sigma_{1}^{2}$ is the strength of the power fluctuation indicator. Assuming that a decision threshold has been chosen, $n_{t h}$, and the bits are equally likely to be transmitted, the bit error rate is provided as [6]

$$
P_{e}=0.5\left[p_{0}\left(x_{e}>n_{t h}\right)+p_{1}\left(x_{e}<n_{t h}\right)\right]
$$


There is an increase in BER as the power fluctuation increases, dependent on atmospheric turbulence [6]. With a large photoelectron count at the receiver along with the detection thermal noise, the generated signal current probability distribution can be approximated as a Gaussian distribution. The optical power can be represented by the optical intensity, $I$ and responsivity, $R$. The received signal current is provided as [6]

$$
i(t)=R I\left[1+\sum_{j=-\infty}^{\infty} d_{j} g\left(t-j T_{b}\right)\right]+n(t)
$$

The probability of error is then given as

$$
P_{e}=Q\left(\frac{i_{t h}}{\sigma}\right)
$$

where $Q(x)=0.5 \operatorname{erfc}(x / \sqrt{2})$

Due to scintillation, an adaptive threshold must be implemented in order to effectively reduce the BER [6]. Due to the inability for coherent combining of the OBS under Rayleigh and Mie scattering in severe outdoor conditions, the remainder of the thesis will consider an outdoor scenario with a large photoelectron count which exhibits a Gaussian distribution.

\subsection{Techniques for OBS}

\subsubsection{Conventional}

With respect to OBS implementations, mechanical pointing and stabilization are still the norm, limiting rapid pointing ability, maintaining a high mechanical complexity, keeping reliability low, and keeping costs high. Non-mechanical approaches to increase pointing speed provide random access pointing, decrease complexity, increase reliability, and reduce cost which can make a major impact on future optical systems. One method of OBS considers the use of a prism. The index of refraction in a prism is larger than that of air, so light travels more slowly within the prism. The angle of light passing through 
a prism will be changed because the light moving through the thick portion of the prism will be delayed compared to light traveling through the thin portion. Accordingly, OBS can be accomplished by changing the thickness of the prism.

Additionally, microelectromechanical (MEMS) mirror devices can be used for OBS as they are free of dispersion and are polarization invariant. Figure 2.1 below shows a tip-tilt MEMS mirror.

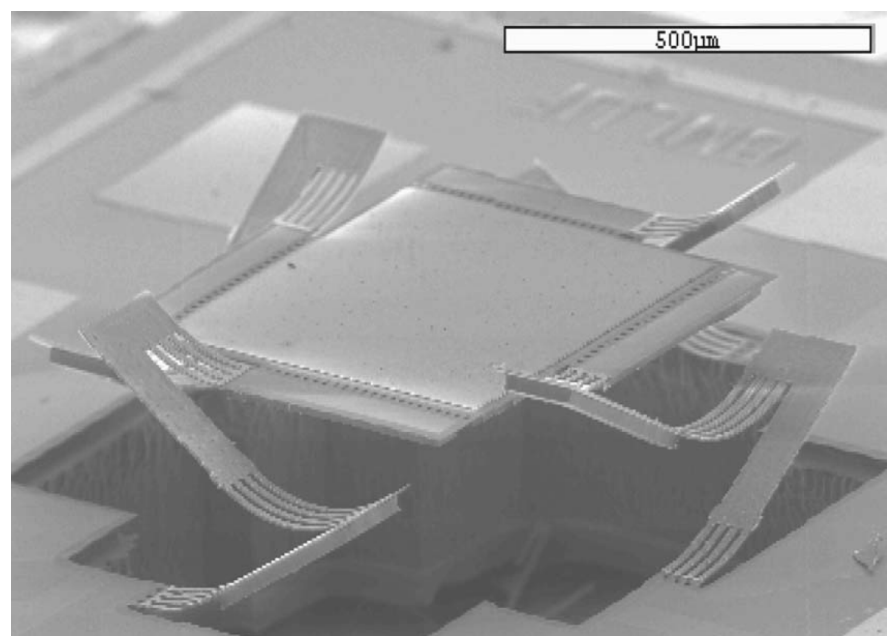

Figure 2.1: MEMS tip-tilt mirror 2

Spatial light modulators (SLMs) are also another alternative which provide OBS capability. By imposing an electric field, it is possible to change the index of refraction of a liquid crystal for light of a given polarization. The birefringence of the liquid crystal is produced by the rotation of the molecules under the applied voltage. Alignment layers create forces to make one orientation preferred in the absence of an electric field. An electric field can cause the molecules to re-orient. The speed of a liquid crystal steering device depends on how fast the liquid crystal molecules will rotate from one orientation to another. The relaxation time for a liquid crystal cell to return to its no-voltage state, once the electric field is removed, is given by [2]

$$
\tau=\frac{t^{2} \gamma}{k_{e} \pi^{2}}
$$


where $\tau$ is the time to return to a no-voltage state. $\gamma$ is the viscosity, $t$ is the cell thickness, and $k_{e}$ is the elastic constant. Figure 2.2 illustrates the SLM functionality.
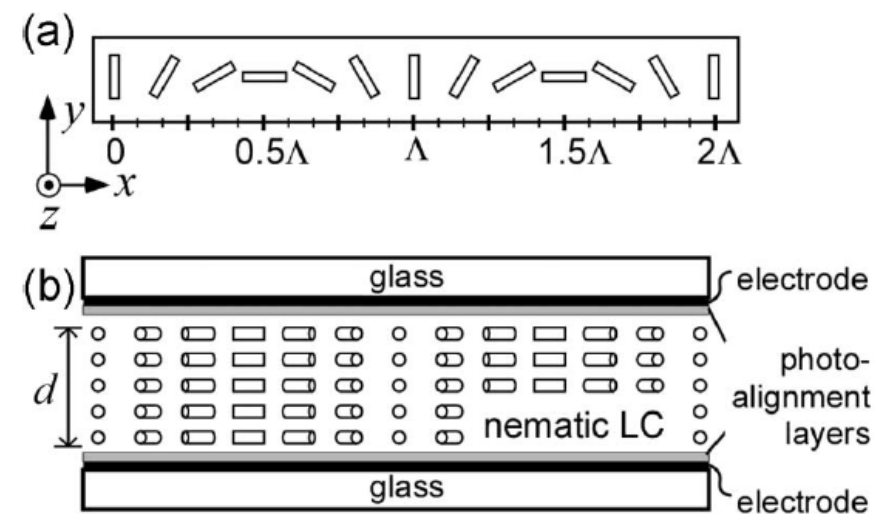

(c)

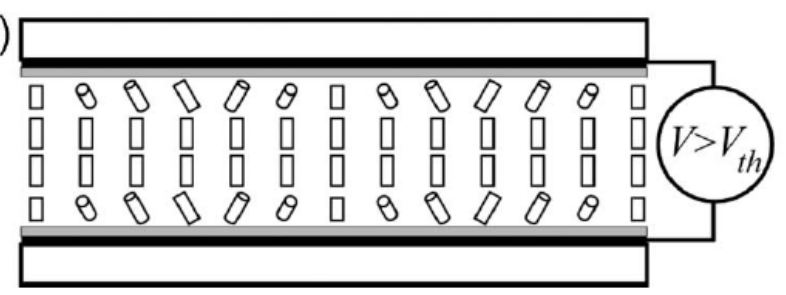

Figure 2.2: SLM functionality 2

Although these OBS implementations are commercially available, they provide very low switching frequency on the order of $\mathrm{kHz}$ and require complex setup and alignment. This makes it unsuitable for our applications. Hence, we will investigate various solidstate monolithic techniques to generate OBS.

\subsubsection{Previous work on solid-state OBS}

In order to determine a starting point for our simulations, it is essential to study prior research which has been done on free-space OBS which involves the use of an OPA. Many OPAs implement the use of different materials for an EOM such as $\mathrm{LiNbO}_{3}$ and silicon. To continue forward, we will study the benefits of each method and proceed with the optimal choice in our simulations. Many studies employ an OPA but transmit in RF to take advantage of the flat group delay that OPAs provide. Hence, the studies that do transmit in the optical domain are limited. Moreover, the studies that do transmit in 
the optical domain use additional signal processing to correct for phase changes, exhibit internal cross-talk, and low steering range. After reviewing the previous work in OPAs, we will outline various improvements which can be considered in order to minimize the internal cross-talk, increase steering range, directivity, and provide a high frequency PLL feedback for phase correction which is not bottlenecked by external processing.

MIT has developed a high speed one-dimensional OBS utilizing an OPA [3]. The steering speed was at $40 \mathrm{MHz}$, a total radiated power of $396 \mathrm{~mW}$, with a full width at half maximum (FWHM) width of $565 \mu \mathrm{rad}$. They achieved OBS by applying $\mathrm{LiNbO}_{3}$ phase modulators. The source was a $100 \mathrm{~mW}$, fiber-coupled DBR laser which was split and sent into 6 phase modulators and then a semiconductor amplifier (SCOWA) array. The parameters are summarized in Table 2.2 below.

Table 2.2: Experimental system parameters

\begin{tabular}{lc}
\hline Parameter & Value \\
\hline Array number & 6 \\
Emitter aperture & $6 \mu \mathrm{m}$ \\
Array pitch & $250 \mu \mathrm{m}$ \\
Fill factor & $1 / 42$ \\
FWHM lobe width & $565 \mu \mathrm{rad}(0.0324$ degrees $)$ \\
Steering range & $4.2 \mathrm{mrad}(0.24$ degrees $)$ \\
\hline
\end{tabular}

Figure 2.3 illustrates their entire system along with a separate controller used to adjust the phase correction of the SCOWA elements [3. It is important to note the phase correction is the main bottleneck of the system which limits the steering speed to around $40 \mathrm{MHz}$. In order to improve the steering speed on the order of $\mathrm{GHz}$, we propose the use of an optical phase locked loop (PLL) which will be introduced in Chapter 4. The phase modulators that were used were commercially available $\mathrm{LiNbO}_{3}$ modulators built by EO space.

$\mathrm{LiNbO}_{3}$ phase modulators and true time delay (TTD) offer significantly faster steering but TTD is preferred as the entire signal bandwidth is steered by the same angle across the signal spectrum with a mean step size on the order of picoseconds. 


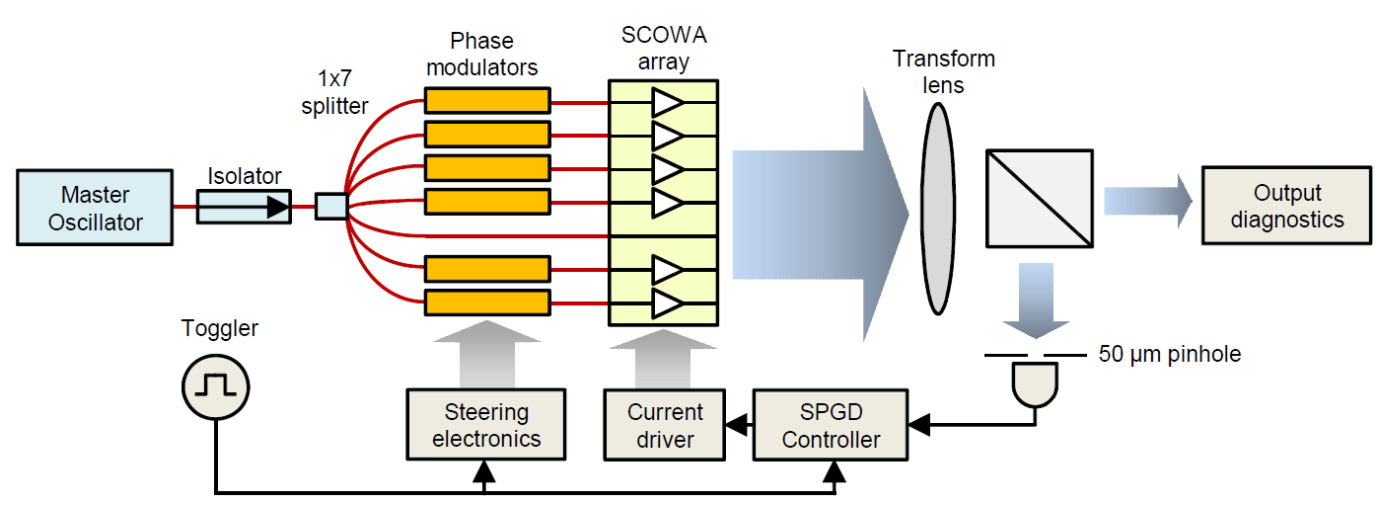

Figure 2.3: OPA developed by MIT [3]

In $\mathrm{LiNbO}_{3}$, if an electric field is applied along the z-axis of the crystal, the refractive index of the material is changed by 5

$$
\Delta n=\frac{1}{2} n_{r}^{3} r_{33} E_{z}
$$

where $r_{33}$ is the electro-optic coefficient for a change of refractive index, $n_{r}$ for light propagating along the z-direction, and $E_{z}$ is the electric field along the z-direction. When a voltage is applied, the total phase shift over an interaction length, $L_{i}$ is 5

$$
\Delta \phi_{0}=\frac{2 \pi \Delta n L_{i}}{\lambda_{c}}=\pi n_{r}^{3} r_{33} \frac{V L_{i}}{d \lambda_{c}}
$$

where $\lambda_{c}$ is the wavelength of the optical signal in a vacuum. The applied voltage and the phase shift exhibits a linear relationship. The voltage required to provide a phase shift of $\pi$ is 5

$$
V_{\pi}=\frac{d \lambda_{c}}{n_{r}^{3} r_{33} L_{i}}
$$

These optical phase shifters can be combined in a parallel array to form an OPA for OBS in free-space. Although $\mathrm{LiNbO}_{3}$ exhibits linear voltage control and high switching frequency, it can not be directly integrated in silicon. Hence, we will now introduce a previous OPA which is completely integrated in silicon. 
UCSB developed a two-dimensional free-space OBS device using an OPA on siliconon-insulator (SOI) [4]. They have demonstrated two-dimensional OBS with a total field of view of $20^{\circ} \times 14^{\circ}$, a beam width of $0.6^{\circ} \times 1.6^{\circ}$ and full-window peak suppression of 10 dB. They implemented 16 channels which were independently tuned. The phase tuning was accomplished thermo-optically by means of resistive heaters in the vicinity of the waveguides. Their system is shown below in Figure 2.4.
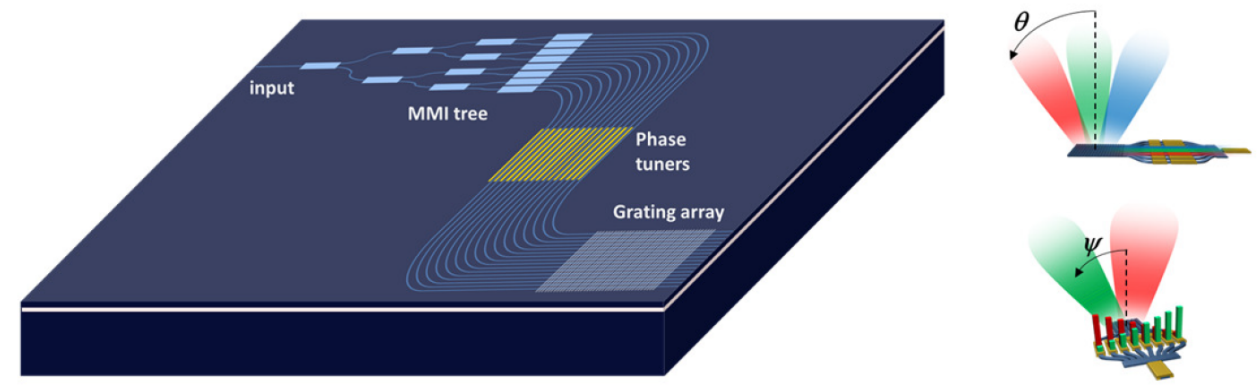

Figure 2.4: Thermal OPA developed by UCSB 4

Materials with a refractive index and a high temperature coefficient of change has the potential to be employed to implement thermo-optic phase tuning. A heating element causes a large refractive index change without optical loss.

Silicon is one waveguide material that is particularly suitable due to its high thermooptic coefficient 13 .

$$
\frac{d n}{d T}=1.84 x 10^{-4} K^{-1} \text { at } \lambda=1.5 \mu m
$$

Here, $n$ is the refractive index of silicon and $T$ is temperature. The thermo-optic phase modulators are implemented by placing heater elements over a section of the waveguides that are connected to the antenna elements. The temperature of the heater is raised by injecting current or applying voltage to the heater elements causing the refractive index of the waveguides to change. Consequently, this causes a change in the phase of the associated waveguide element within the array factor.

Although the method of thermo-optic modulators is effective at producing a shift in phase in silicon, it produces significant internal cross-talk which must be accounted for. 
Additionally, the use of an external processor must be used as a feedback to correct for the thermal cross-talk. Our proposed method of an optical PLL in Chapter 4 will omit the need of a processor as it will directly correct the phase by means of a heterodyne down conversion.

\subsubsection{Silicon phase modulation}

To conclude from the work done by MIT and UCSB, we will propose the use of doped silicon as the EOM instead of $\mathrm{LiNbO}_{3}$ or thermo-optic modulators. This will produce a monolithic design as well as minimize the internal cross-talk from thermo-optic heaters. To introduce a potential silicon EOM for use in an OPA, a sample integrated SOI phase modulator is shown in Figure 2.5. The dimensions are on the order of microns.

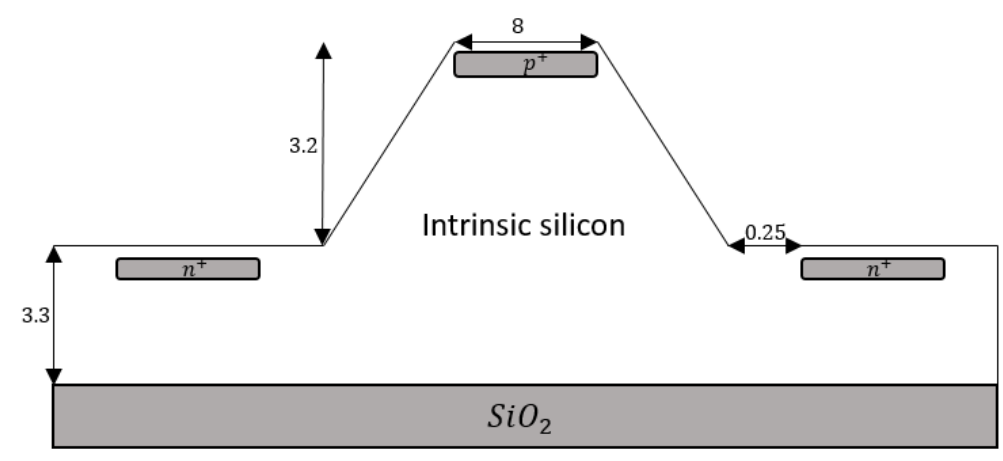

Figure 2.5: Integrated SOI phase modulator [5]

The electro-optic effect known as the Pockels effect which is applicable to $\mathrm{LiNbO}_{3}$ modulators, is not applicable to silicon due to the geometry of its crystal structure, hence it will not produce optical modulation. However, the Kerr effect is present in silicon and reflects the following change in the refractive index as follows [5]:

$$
\Delta n=s_{33} n_{0} \frac{E^{2}}{2}
$$

where $s_{33}$ is the Kerr coefficient, $n_{0}$ is the unperturbed refractive index and $E$ is the applied electric field. Since the Kerr effect has a minimal effect, a change of $10^{-4}$ in refractive index with an applied field of $100 \mathrm{~V} / \mu \mathrm{m}$. At a wavelength around $1 \mu \mathrm{m}$, the 
Franz-Keldysh effect is more prominent where absorption occurs when the wavelengths are close to the bandgap. Although it requires less applied field, $20 \mathrm{~V} / \mu \mathrm{m}$ to produce the $10^{-4}$ change in refractive index, its effect decreases significantly as the wavelength approaches infrared. A more effective way to produce an increased change in refractive index with an applied field is by means of injection or depletion of carriers in silicon. At $\lambda_{0}=1.55 \mu \mathrm{m}$ :

$$
\Delta n=\Delta n_{e}+\Delta n_{h}=-8.8 \times 10^{-22} \Delta N_{e}+8.5 \times 10^{-18}\left(\Delta N_{h}\right)^{0.8}
$$

where $\Delta n_{e}$ is the change in refractive index resulting from change in free electron carrier concentrations, $\Delta n_{h}$ is the change in refractive index resulting from change in free hole carrier concentrations. $N_{e}$ and $N_{h}$ is the electron and hole carrier concentrations. With higher doping levels, higher refractive index is expected.

From Equation 2.15, we can see that an effective change in refractive index can be accomplished by doping silicon. This makes doped silicon a feasible monolithic EOM instead of previously implemented $\mathrm{LiNbO}_{3}$ or thermo-optic SOI phase modulators. For the remainder of the thesis, we will focus on the passive design of the silicon OPA as the EOM. 


\section{Chapter 3}

\section{Optical Shadowing}

In this Chapter, we will present our novel characterization of shadowing in an OWC channel. Since shadowing is a unique scenario in the optical domain as opposed to the RF domain, our characterization of shadowing further supports the use of OBS as the only possible solution to increase the SNR and reduce the BER in an OWC link. MATLAB $^{\text {TM }}$ software was used to execute the simulation of shadowing.

Every wireless communication system design must take into account three major channel propagation impairments: short-term (multipath) fading, and long-term fading (shadowing). Shadowing is the effect when the received signal power fluctuates due to objects obstructing the propagation path between the transmitter and receiver. These fluctuations are experienced on local-mean powers, that is, short-term averages that remove multipath fading fluctuations [14]. RF technology is fairly robust to blocking and shadowing due to the propagation nature of RF EM waves, although at higher carrier frequencies, RF requires LOS transmission and shadowing becomes a more prominent

issue. Hence composite fading/shadowing models such as Generalised-K and joint fading and two-path shadowing (JFTS) are used to evaluate system performance under unique conditions but can not be directly applied to OWC systems.

Due to the nature of the optical spectrum, shadowing is a significant issue especially over short distances and small FOVs [15]. This can impair communications in both 
quasi-stationary and fast varying outdoor (vehicle to vehicle) environments. However, by employing diffused transmitters with a wide FOV as well as effectively utilizing the diffraction phenomenon of light, the issues caused by shadowing can be alleviated. Diffused systems enable the user terminals to have a wide degree of mobility at the expense of a high path loss. The diffused channels however suffer not only from optoelectronic bandwidth constraints but also from low-pass multipath distortion. It is important to note that unlike RF wireless channels, diffuse optical channels do not exhibit fading with intensity modulation. This is due to the fact that the PD at the receiver integrates the optical intensity field over an area of millions of square wavelengths and no change in the channel response is noted if the PD is moved a distance on the order of a wavelength. Therefore, the large size of the PD relative to the wavelength of light provides a degree of spatial diversity which eliminates multipath fading [16]. In outdoor OWC, multiple reflections are not prominent therefore multipath induced signal distortion is not of a great concern.

An opaque object can partly shadow or completely block an optical link because infrared light does not penetrate opaque objects. In order to ease the pointing and shadowing problems of point-to-point links, diffuse transmitters radiate optical power over a wide solid angle. Hence, the transmitter does not need to be aimed directly at the receiver. This allows a wide degree of mobility for user terminals, at the expense of a high path loss. In general, this is a major limitation of the OWC link and can't be avoided. Many studies have recommended the use of multiple-input-multiple-output (MIMO) to overcome shadowing and employing diversity techniques but this is still limited to its spatial configuration.

To further increase the diversity of the OWC channel, we are investigating the use of OBS at the transmitter side to mitigate significant issues such as shadowing and high path loss. Essentially, OBS has the capability to increase SNR and decrease the BER over an AWGN channel. 


\subsection{Channel modelling with shadowing}

For the following sections, we considered the use of an LED source in order to illustrate the effect of shadowing. The aim of this section is to study the effect of shadowing on the received signal and find its PDF. In a typical VLC setup shown in Figure 3.1, two terms related to the light brightness can be defined, the illuminance and the luminance. The illuminance indicates the intensity of light that is travelling towards a surface while luminance means the brightness of that illuminated surface [17]. The illuminance at an angle $\phi$ from the radiating axis is given by,

$$
I(\phi)=I(0) \cos ^{n}(\phi)
$$

where $I(0)$ is the peak illuminance perpendicular to the surface of the radiating element, $\phi$ is the angle of irradiance measured from the axis and $n$ is the order of the radiating source. Note, in general, $n$ is given by,

$$
n=\frac{-\ln (2)}{\ln \left(\cos \left(\phi_{1 / 2}\right)\right)}
$$

where $\phi_{1 / 2}$ is the semi-angle at half illuminance [18]. In this work, it is assumed that an LED has a Lambertian radiation pattern [19], which means $n=1$. That is, the half power angle $\phi_{1 / 2}$ is 60 degrees for an LED indicating wide angle radiation as shown in Figure 3.1 .

To mitigate the effect of shadowing, spatial diversity is commonly implemented where multiple transmitters and receivers are deployed around the shadowed area [20]. However, having such a setup is not always guaranteed and sustainable, henceforth it is necessary to study the effect of shadowing without having spatial diversity. This is necessary to effectively design a OWC system, along with obtaining the parameters that control its performance determined by the signal error probability under various shadowing con- 


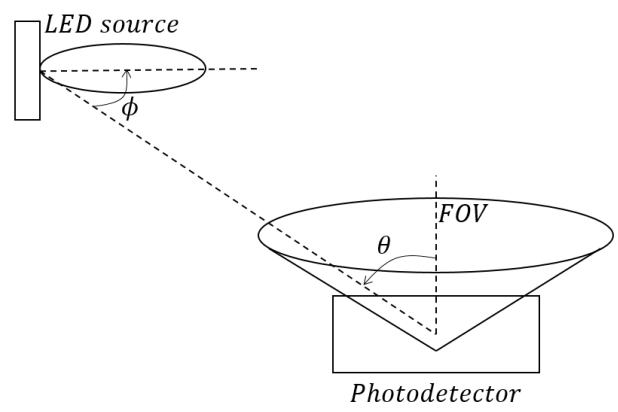

Figure 3.1: VLC system setup

ditions. In this work, we are interested in the cases of low (or absent), moderate and severe shadowing. In a typical VLC system incorporating an LED, the transfer function between the transmitter and the receiver is given by 21],

$$
H=\frac{\left(\frac{n+1}{2 \pi}\right) A_{r} \cos ^{n}(\phi) \cos (\theta)}{R_{d}^{2}}
$$

where, $n$ is the order of the source, $\phi$ is the emitting angle from the LED to the PD, $\theta$ is the incident angle from the LED to the $\mathrm{PD}, R_{d}$ is the distance between the lighting source and the surface of the detector, and $A_{r}$ is the active area of the PD. The channel impulse response for a bimodal obstruction is,

$$
\begin{aligned}
& h_{1}(t)=\frac{\left(\frac{n+1}{2 \pi}\right) A_{r} \cos ^{n}(\phi) \cos (\theta)}{R_{1}^{2}} \delta\left(t-\frac{R_{1}}{c}\right) \\
& h_{2}(t)=\frac{\left(\frac{n+1}{2 \pi}\right) A_{r} \cos ^{n}(\phi) \cos (\theta)}{R_{2}{ }^{2}} \delta\left(t-\frac{R_{2}}{c}\right)
\end{aligned}
$$

where, $R_{1}$ and $R_{2}$ are the total distance from the lighting source to the receiver, taking into account the light diffraction around the obstructing object from the first edge and the second edge of the obstructing object, respectively as illustrated in Figure 3.2 [18. 


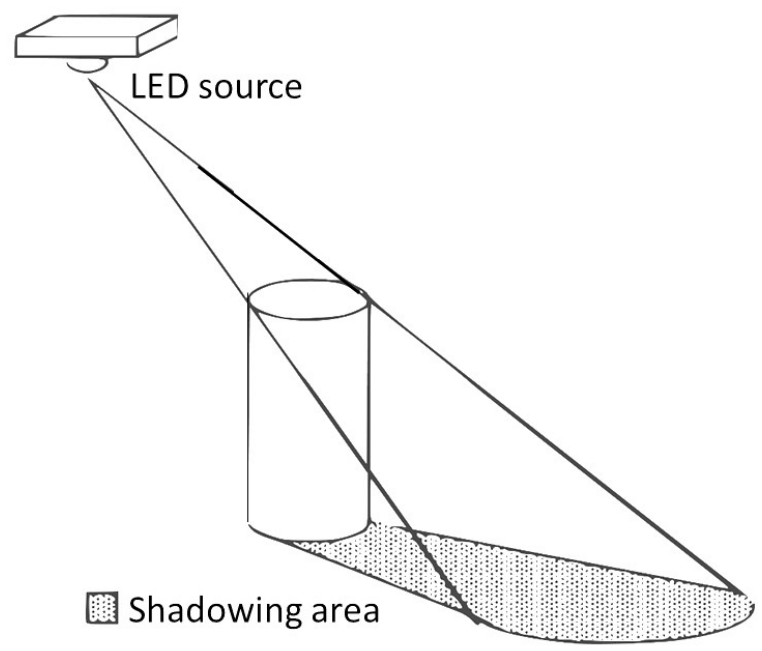

Figure 3.2: LED shadowing example

The received signal energy $E_{r}$ is,

$$
E_{r}=\int_{0}^{T}\left[x(t) *\left(h_{1}(t)+h_{2}(t)\right)\right]^{2} \mathrm{~d} t .
$$

where $x(t)$ is the transmitted signal, and $T$ is the overall bit duration.

\subsection{Shadowing effect}

In a standard OWC system without shadowing, with a significant photoelectron count, it can be assumed that the transmitted signal encounters AWGN, hence the distribution at the receiver remains Gaussian. Since shadowing plays a significant role in OWC, the final distribution will result in a multi-modal distribution depending on the severity of the shadowing effect as well as the complexity of the obstructing object (i.e. non uniform objects with complex cavities cause secondary interference effects thereby varying the channel gain) but we will only consider a bimodal distribution here for simplicity. Although, the PD receives a single voltage, we can assume a time-varying approximation.

As apparent in Figure 3.2 (assuming light only passes over two edges), we can assume 
a bimodal power distribution. With a bimodal approach, and assuming the PD receiver holds a large FOV, the optical power will be received from two distinct edges passing the object, each with different polarization effects. Therefore, depending on the severity/complexity of the object surface, each polarizing path passing the object will appear as a local maximum. Since we are considering the simple case of light passing only from two sides of the object, this can be represented as a bimodal distribution. The separation of this distribution in a time varying environment can be represented by two means and variances 22 by,

$$
S(t)=\frac{\mu_{0}(t)-\mu_{1}(t)}{2\left(\sigma_{0}(t)+\sigma_{1}(t)\right)}
$$

where, $\mu_{1}>\mu_{0}$. Here, $S(t)$ measures the distance between the means of the two normal functions which is roughly the distance between the two peaks [22]. The Bimodal distribution has the following PDF 23]

$$
\begin{aligned}
f\left(z, \mu_{0}, \sigma_{0}, \mu_{1}, \sigma_{1}\right)= & \frac{1}{2 \sqrt{2 \pi\left(\sigma_{0}\right)^{2}}} \exp \left(\frac{-\left(z-\mu_{0}\right)^{2}}{2\left(\sigma_{0}\right)^{2}}\right) \\
& \quad+\frac{1}{2 \sqrt{2 \pi\left(\sigma_{1}\right)^{2}}} \exp \left(\frac{-\left(z-\mu_{1}\right)^{2}}{2\left(\sigma_{1}\right)^{2}}\right)
\end{aligned}
$$

where $\mu_{0}, \sigma_{0}, \mu_{1}, \sigma_{1}$ are the means and the standard deviations of the first and second combined Gaussian distributions respectively.

Notice that when $S(t)$ decreases with a uniform ideal obstruction, the distribution will approach a standard Gaussian distribution and the received power will contain only a single maxima. 


\subsection{Probability of error}

In short-distance LOS links, multipath dispersion is insignificant. Hence, LOS links are often only modelled with a linear attenuation and delay [24. Optical LOS links are considered as non-frequency selective where the path loss depends on the square of the distance between the transmitter and the receiver. We will now consider the error probability derivation with shadowing effect under a bimodal distribution case assuming OOK is employed. This can, however, be extended to a multi-modal case.

To derive the average probability of error, $P e_{\text {avg }}$ of the received signal for the three cases of shadowing: mild or absent-shadowing, moderate and severe shadowing, we will consider Figures 3.3, 3.4, 3.5 and 3.6 to illustrate their effect. We present a closed formula for the probability of error for each case of shadowing along with the derivation for the first case. The other cases can be derived using a similar procedure. It is important to note that in the case of absent or mild shadowing, the transmitted light will be received without being significantly deteriorated by the shadowing object, thus, the received signal will be modeled as a Gaussian distribution for both cases of transmitting symbols represented by $s_{1}$ and $s_{0}$, with average values $\mu_{0}, \mu_{1}$ respectively as illustrated in Figure 3.3 .

In the case of having moderate or severe shadowing, light will be more affected by the shadowing object, hence the signal will be received from both sides which will result in a bimodal distribution. This is clearly depicted in Figures 3.4, and 3.5, where two Gaussian distributions are created for each received symbol, thereby resulting in a bimodal distribution. The average expected received values can be represented by four vertical lines, with two possible average values for the case of receiving $s_{1}$, represented with average values $\mu_{1}, \mu_{1}^{\prime}$ and two possible average values for the case of receiving $s_{0}$ represented by $\mu_{0}, \mu_{0}^{\prime}$. However, considering the fact that the shadowing is moderate, the two average values of receiving $s_{0}$ are close to each other which also applies for the case of receiving $s_{1}$. Therefore, we have two decision regions for the probability of error calculation. With respect to severe shadowing, the average values of the two modes are shifted significantly from each other. In this case we assume the shift is large enough to 
approximate the error by representing each mode as a normal distribution. We assumed that when the separation, $S(t)>1$ between modes, the distribution becomes two normal distributions as shown in Figure 3.3. Moreover, in a severe shadowing case, the Gaussian distribution of $s_{0}$ and $s_{1}$ become close to each other which significantly affects the probability of error which will be verified in the simulation section.

Furthermore, due to the obstruction effects, we will assume that the width of each Gaussian distribution is halved when compared to the case of no shadowing due to the transmitted signal power being distributed between the two paths around the obstructing object. In the following, we derive the average probability of error, $P e_{\text {avg }}$

$$
P e_{\text {avg }}=P_{e 0} P_{0}+P_{e 1} P_{1}
$$

where $P_{0}, P_{1}$ are the a priori probability of sending each symbol with respective error probabilities, $P_{e 0}$, and $P_{e 1}$. Due to symmetry, one can conclude that $P_{e 0}=P_{e 1}=P_{e}$. We define the decision region as

$$
x_{0}=\frac{\mu_{0}+\mu_{1}}{2}
$$

where $x_{0}$ is the mid-point between the two means. The $P e$ is defined as

$$
P_{e}=\int_{x o}^{\infty} \frac{1}{\sqrt{2 \pi \sigma^{2}}} \exp \left(\frac{-\left(x-\mu_{1}\right)^{2}}{2 \sigma^{2}}\right) \mathrm{d} x
$$

Recall the $Q$ function as

$$
Q_{x}=\int_{x o}^{\infty} \frac{1}{\sqrt{2 \pi}} \exp \left(\frac{-y^{2}}{2}\right) \mathrm{d} y
$$


let $y=\frac{x-\mu_{0}}{\sigma}$ then $d y=\frac{d x}{\sigma}$,

$$
P_{e}=P_{r}\{X>x o\}=P_{r}\left\{y>\frac{x_{0}-\mu_{0}}{\sigma}\right\}
$$

By changing the integration limits, from equations (3.13), we will obtain $P e$ as:

$$
P_{e}=\int_{\left(\frac{x_{0}-\mu_{0}}{\sigma}\right)}^{\infty} \frac{1}{\sqrt{2 \pi}} \exp \left(\frac{-y^{2}}{2}\right) \mathrm{d} y
$$

then

$$
P_{e}=Q\left(\frac{x_{0}-\mu_{0}}{\sigma}\right)=Q\left(\frac{\frac{\mu_{0}}{2}+\frac{\mu_{1}}{2}-\mu_{0}}{\sigma}\right)
$$

Equation (3.15) can be simplified to

$$
P_{e}=Q\left(\frac{\mu_{1}-\mu_{0}}{2 \sigma}\right)
$$

To find the probability of error for moderate and severe shadowing when intensity modulation is employed, the probability of error will be based on 4 average values due to the bimodal distribution as shown in Figure 3.4 and Figure 3.5. Then by applying the same procedure as shown above and by analysis of Figure 3.4 and Figure 3.5 , the probability of error for moderate and severe shadowing can be given by Eq. (3.17) and Eq. (3.18) respectively. 


$$
\begin{gathered}
P e_{\text {avg }}=2\left(Q\left(\frac{\mu_{1}-\mu_{0}}{4 \sigma}\right)+Q\left(\frac{\mu_{1}^{\prime}-\mu_{0}}{4 \sigma}\right)\right) \\
P e_{\text {avg }}=4\left(Q\left(\frac{\mu_{1}^{\prime}-\mu_{0}^{\prime}}{4 \sigma}\right)+Q\left(\frac{\mu_{0}-\mu_{1}^{\prime}}{4 \sigma}\right)+Q\left(\frac{\mu_{1}-\mu_{0}}{4 \sigma}\right)\right)
\end{gathered}
$$

Notice that in the case of moderate shadowing, we will still have two decision regions for detecting $s_{1}$ and $s_{0}$, while in the case of severe shadowing, the error probability is further increased as shown below due to the crossing of decision regions for detecting $s_{1}$ or $s_{0}$ which results in four decision regions.

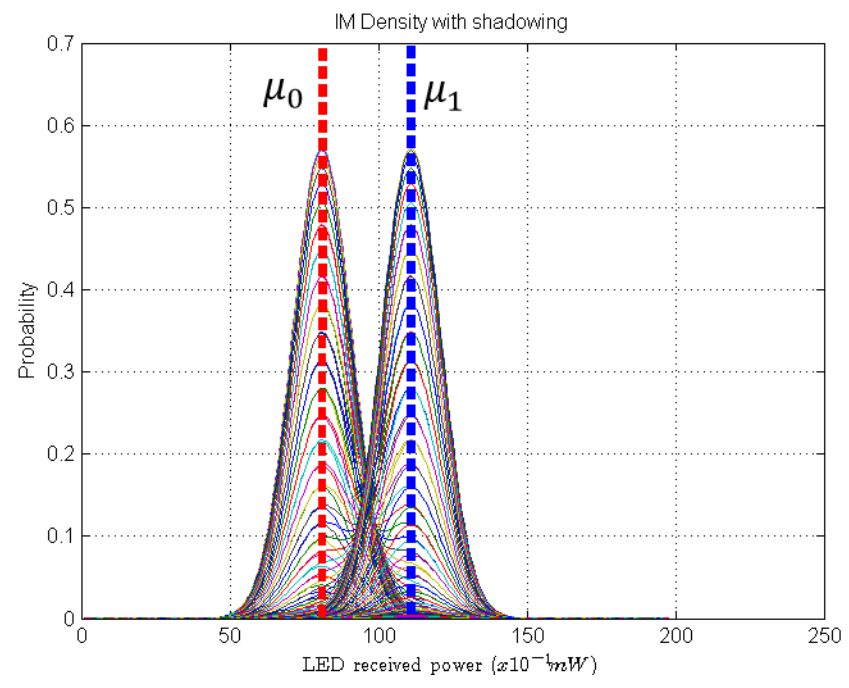

Figure 3.3: LED-mild shadowing 


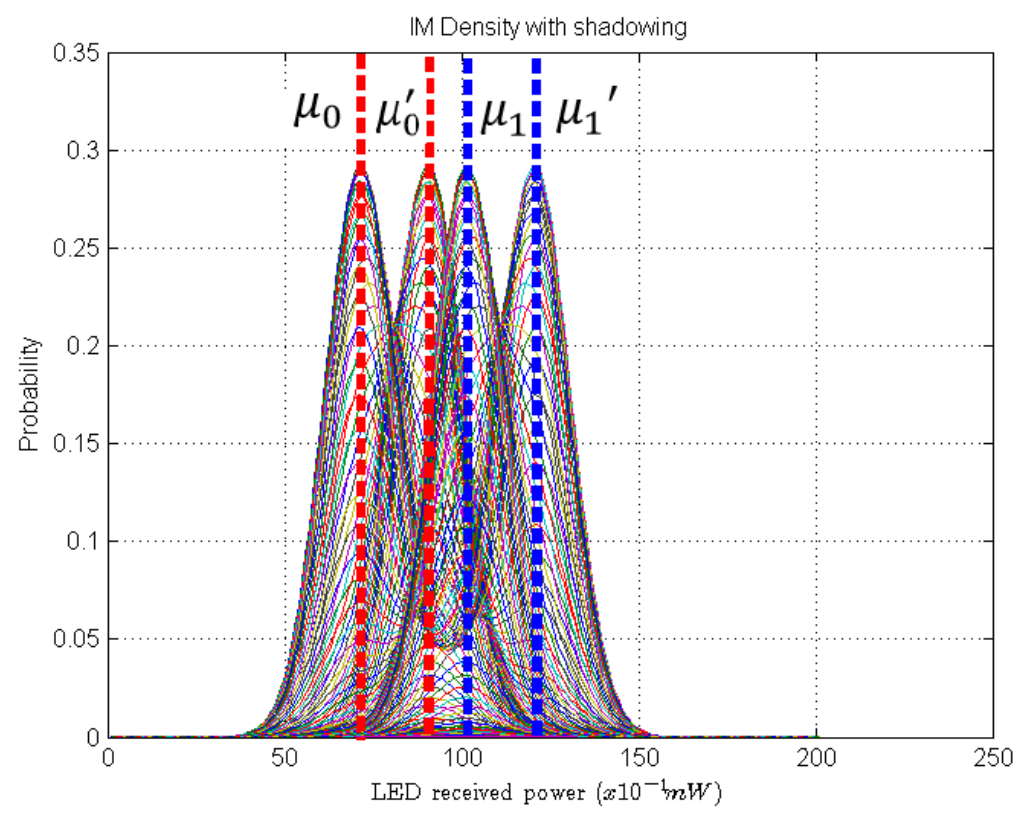

Figure 3.4: LED-moderate shadowing

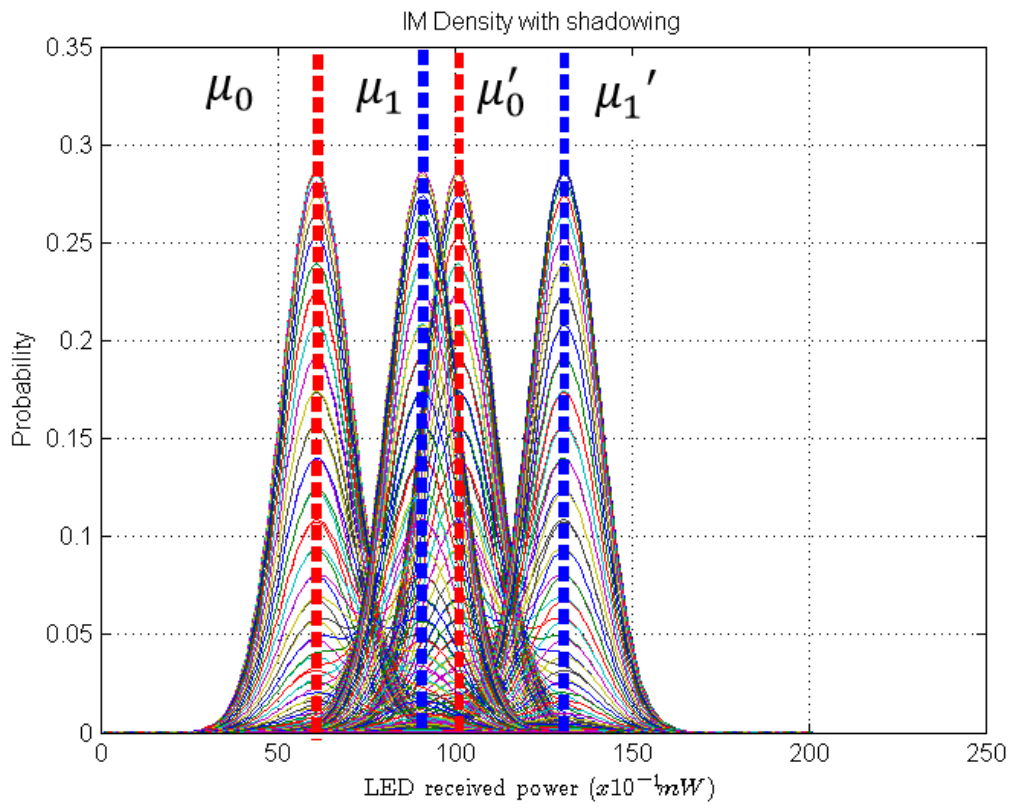

Figure 3.5: LED-severe shadowing 


\subsection{Shadowing simulations}

We assumed both Gaussian distributions of receiving $s_{1}$ or $s_{0}$ have equivalent variances but different means. Additionally, we are assuming intensity modulation is employed in the system where a stationary object with two main edges is obstructing the LOS signal, hence each modulation level will exhibit two modes. The error significantly increases when shadowing is in effect. Figures 3.343 .5 illustrate the density functions with the effects of shadowing where blue and red lines represent modulation levels for $s_{1}$ and $s_{0}$, respectively.

Figure 3.3 illustrates a clear distinction between each modulated intensity level without exhibiting dual modes, hence there may be an obstruction with a uniform surface resulting in low shadowing or even absent-shadowing. Figure 3.4 clearly shows the bimodal shadowing effect as there are two modes for each modulated intensity level. In Figure 3.5, the overlap between the respective modes resulting from severe shadowing become apparent. It is shown that the respective intensity levels begin to overlap. Hence, the decision region of both symbols significantly overlaps thereby greatly increasing the BER.

To simulate the probability of error for different cases of shadowing, we used MATLAB ${ }^{\mathrm{TM}}$ by varying the received signal energies as well as its variances for the bimodal distribution. The simulation results clearly show that the error probability significantly increases due to the interleaving signal modes with each respective modulation. Figure 3.6 is the result of our simulation which compares the error probabilities with different shadowing effects. Clearly, the severe shadowing has the worst error probability when compared to the low and moderate shadowing cases. It is also clear that for all shadowing cases, when the received signal power is increased, as a result of increasing the transmitted power, the probability of error decreases. 


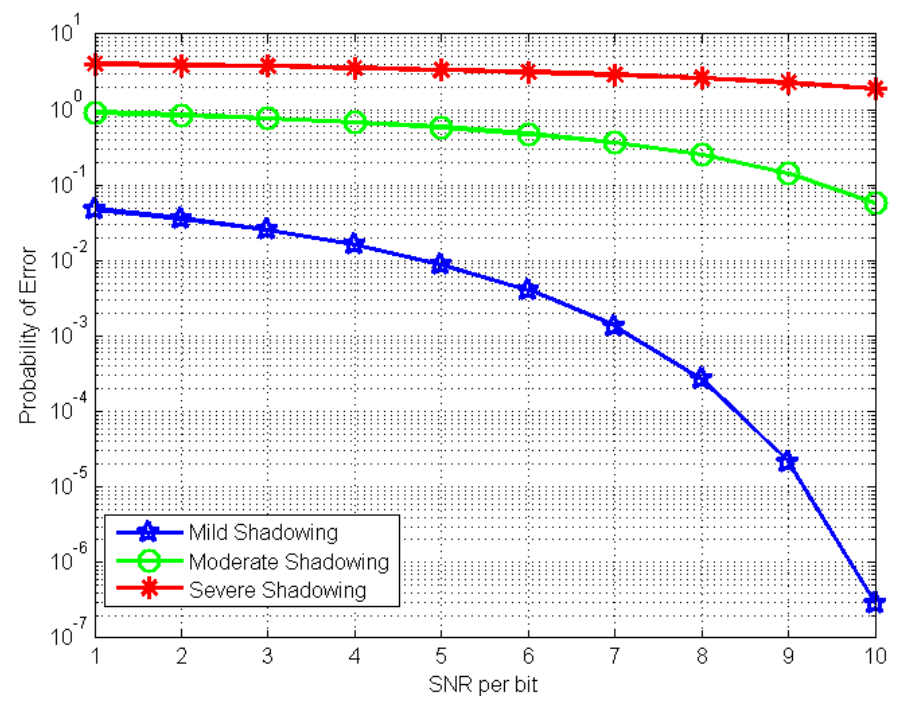

Figure 3.6: Error probability with different shadowing effects

It has been shown that the effect of shadowing on a OWC system is detrimental and can completely deteriorate a communication link. From our studies, a two-edged obstructing object with uniform surface results in a bimodal distribution of the received power. With more severe and complicated shadowing, multi-modal reception appears as shown in Figure 3.7. The use of OBS is the only possible way to mitigate object-induced shadowing.

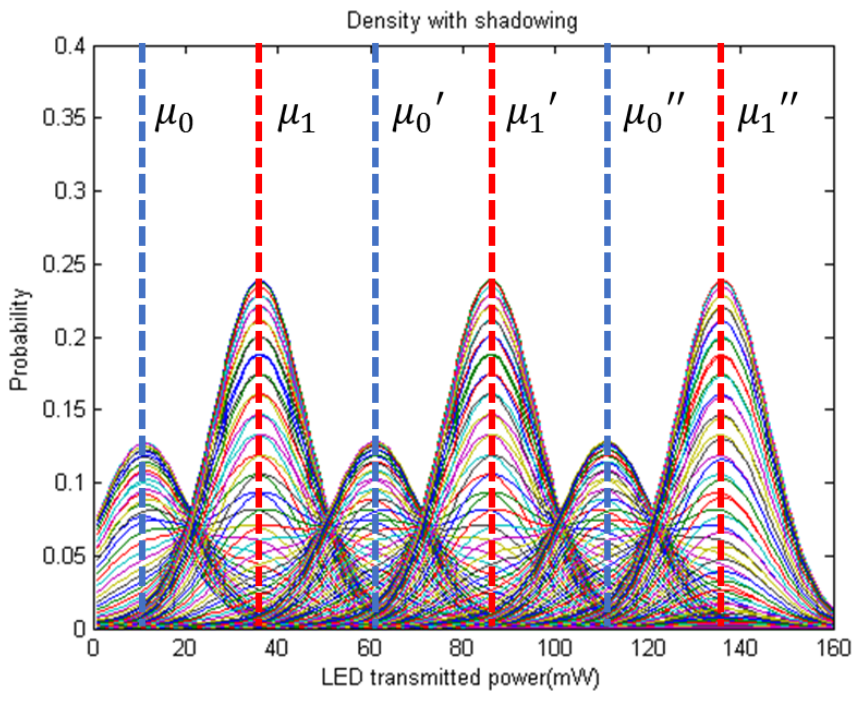

Figure 3.7: Multimode scenario 


\section{Chapter 4}

\section{Monolithic Optical Phased Array}

In this Chapter we will present the steps required to design a monolithic SOI OPA for OBS. We will first present the equations which characterize the beam forming in freespace that represents the MEEP ${ }^{\mathrm{TM}}$ simulations in Chapter 5. Then we will present the conditions which ensure single-mode propagation in the waveguide. Moreover, we will present our proposal of the optical PLL to mitigate phase disturbances. Finally, for future applications, we will investigate the effect and requirements of using an LED instead of a LD source in the OPA.

The far-field radiation pattern of optical array antennas is described by a number of factors, the most important of which is the array factor as shown below 25]. The array factor accounts for the effect of the array geometry independent of the radiation.

$$
T(\theta, \phi)=\sum_{m=0}^{M-1} \sum_{n=0}^{N-1} A_{m n} e^{-j \beta_{m n}} e^{K S_{m n}}
$$

where $K$ is the wave vector, $A_{m n}$ is the amplitude, and $\beta_{m n}$ is the phase of the signal received element indexed by $(n, m)$ with position vector $S_{m n}$.

$$
K S_{m n}=k_{0}\left(m \Lambda_{x} \sin (\theta)+n \Lambda_{y} \sin \psi\right)
$$


The phase delay for a structure with a waveguide delay section of length $\Delta L_{m n}$ is

$$
\beta_{m n}=n_{e f f} \frac{2 \pi}{\lambda} \Delta L_{m n}
$$

where $n_{\text {eff }}$ is the effective index for the TE-like fundamental mode of the waveguide. It indicates that the change in wavelength brings a change in the relative phase of the adjacent elements: a process referred to as phase tuning. This results in OBS if the delay is

$$
\Delta L_{m n}=m \Delta L
$$

The angles and the rate of change of the steered angle in $\theta$ at which the peaks in the array factor occur can be obtained from where $\Lambda$ is the spacing between elements and $q$ is the order of the delay:

$$
\begin{gathered}
\sin \theta=\frac{q \lambda}{\Lambda}+n_{\text {eff }} \frac{\Delta L}{\Lambda} \\
\frac{d \sin \theta}{d \lambda}=\frac{q}{\Lambda}+\frac{d n_{e f f}}{d \lambda} \frac{\Delta L}{\Lambda}
\end{gathered}
$$

We have seldom seen OPA methods which are purely monolithic as they typically employ the use of external modulators. Most SOI OPAs use thermally controlled phase modulators but this suffers from thermal cross-talk and must be compensated accordingly. Hence, it is desirable to design a purely SOI monolithic OPA which incorporates a fully integrated phase modulator.

\subsection{Waveguide conditions}

The LD or optical source must be coupled to a planar waveguide which requires single mode operation. A planar waveguide should follow the following conditions to ensure 
single mode operation [5],

$$
\begin{gathered}
\theta_{c}<\cos ^{-1}\left(\frac{\lambda_{0}}{2 n_{1} h}\right) \\
h<\left(\frac{\lambda_{0} / 2}{n_{1} \sqrt{1-\left(n_{2} / n_{1}\right)^{2}}}\right)
\end{gathered}
$$

where $h$ is the waveguide height, $\theta_{c}$ is the critical angle, $\lambda_{0}$ is the operating wavelength, $n_{2}$ and $n_{1}$ are the respective refraction indices relevant to SOI. For example, for a single mode operation in an SOI waveguide at a wavelength of $1.3 \mu \mathrm{m}, h$ must be less than 0.2 $\mu \mathrm{m}$.

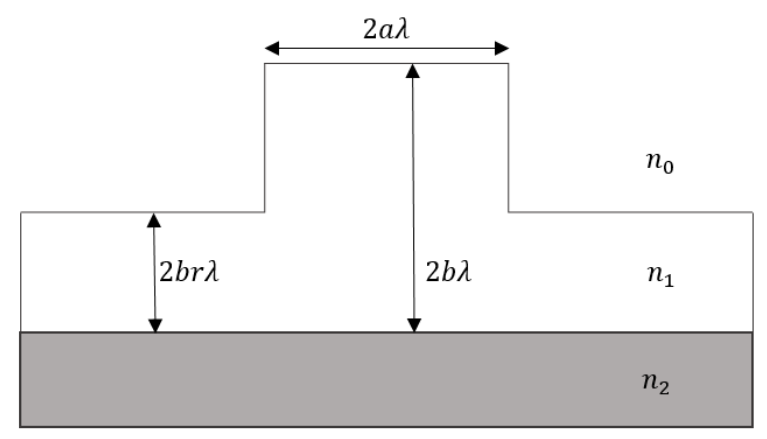

Figure 4.1: Planar waveguide geometrical constraints

The following condition for the aspect ratio must be followed to restrict the geometry of the rib waveguide to single-mode operation [5] as illustrated in Figure 4.1.

$$
\frac{a}{b} \leq 0.3+\frac{r}{\sqrt{1-r^{2}}}
$$

\subsection{OPA with feedback}

The phase modulator components can be fully integrated along with the planar waveguide to create a fully monolithic OPA as shown below. Finally, after coupling a LD to the planar waveguide, it is split into multiple branches which are individually phase 
modulated.

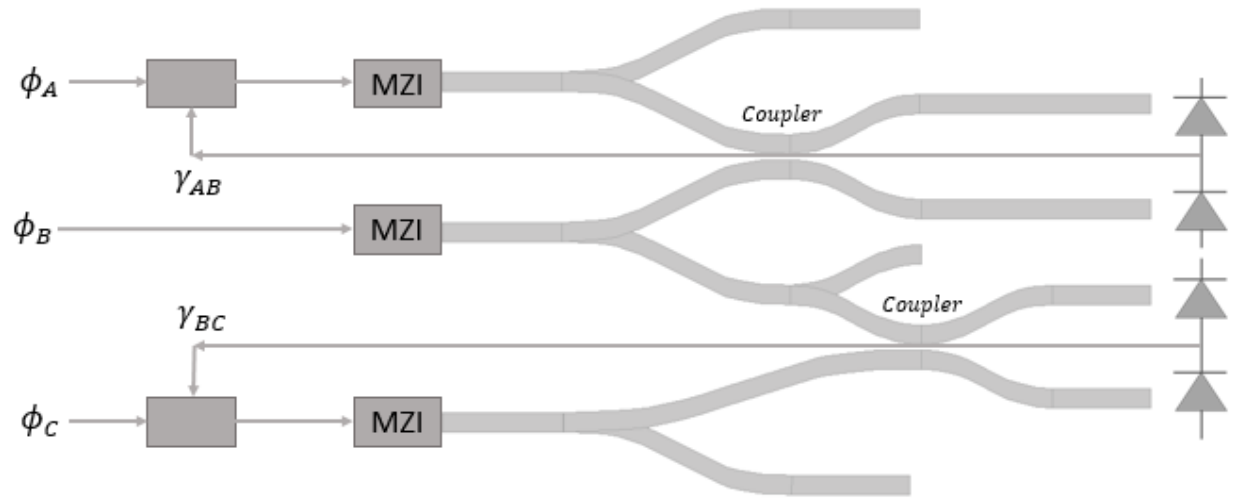

Figure 4.2: Internal OPA feedback

Any thermal or vibrational disturbances can cause a shift in phase in each branch, hence an internal optical phase-locked-loop (PLL) has been included, shown as the termination between each branch. The termination could detect the amplitude of the interference of both branches to effectively determine the phase difference between the branches in order to correct for any phase disturbances as illustrated in Figure 4.2 .

Alternatively, each branch can be connected and coupled to a local-oscillator (LO) laser [25]. After coupling with the secondary laser, it can be attached to a PD which acts as a square-law device to down convert the optical frequency and detect the phase shift. The EM field in each branch is provided as

$$
\begin{gathered}
E_{1}(t)=\left[A_{1}+n_{1}(t)\right] e^{j \omega_{1} t} \vec{x} \\
E_{2}(t)=\left[A_{2}(t) e^{j \phi_{2}(t)}+n_{x}(t)\right] e^{j \omega_{2} t} \vec{x}+n_{y}(t) e^{j \omega_{2} t} \vec{y}
\end{gathered}
$$

where $A_{2}(t)$ and $\phi_{2}(t)$ are the modulated amplitude and phase of the transmitted signal, respectively. $\omega_{2}$ is the carrier frequency of the signal, $\vec{x}$ and $\vec{y}$ is the polarization of the signal. $n_{x}(t)$ and $n_{y}(t)$ are noise in the polarization directions. $A_{1}, n_{1}(t)$, and $\omega_{1}$ is the continuous wave amplitude of LO laser, the noise of the LO laser, and the angular 
frequency, respectively. Finally, the photocurrent of the PD is

$$
i(t)=\frac{R}{2}\left|E_{1}(t)+E_{2}(t)\right|^{2}+i_{s h}+i_{t h}
$$

where $R$ is the responsivity of the $\mathrm{PD}, i_{s h}$ is the photocurrent shot-noise, $i_{t h}$ is the thermal noise of the receiver. The $\mathrm{PD}$ responsivity is equal to

$$
R=\eta \frac{2 \pi e}{h \omega_{c}}
$$

where $e=1.6 x 10^{-19} C$ is the charge per electron, $h \omega_{c}$ is the energy per photon, $\hbar=$ $6.63 \times 10^{-34} \mathrm{~J}$ is Plancks constant, and $\eta$ is the quantum efficiency of the PD. Furthermore, the photocurrent expands out to

$$
\begin{array}{r}
i(t)=\frac{R}{2}\left[\left|A_{1}+n_{1}(t)\right|^{2}+\left|A_{2}(t) e^{j \phi_{2}(t)}+n_{x}(t)\right|^{2}+\left|n_{y}(t)\right|^{2}\right] \\
+R A_{1} A_{2}(t) \cos \left[\omega_{I F}(t)+\phi_{2}(t)\right]+ \\
R * \operatorname{Re}\left[A_{1} n_{x}(t)+A_{2}(t) e^{j \phi_{2}(t)} n_{1}(t)\right] e^{j \omega_{I F} t}+i_{s h}+i_{t h}
\end{array}
$$

After coupling the two laser elements and down converting by means of a PD, the initial frequencies of $\omega_{1}$ and $\omega_{2}$ down convert to a much lower intermediate frequency, $\omega_{I F}$. This intermediate frequency will be almost DC as the secondary branch is frequency locked to the primary branch. Hence this is a heterodyne down conversion with coherent retrieval of the modulated phase, $\phi_{2}(t)$. From this point on, an electronic PLL can be implemented without the need for additional processing based on the feedback from the phase at the intermediate frequency. This proposed method of ours significantly improves the steering range by allowing high frequency internal phase correction as opposed to previous techniques which are bottlenecked by a signal processor. 


\subsection{Optical source}

\subsubsection{LED vs Laser coherence}

Although LDs are ubiquitous in monolithic OPA applications, we will investigate the use of an LED source for future OBS applications. It is well known that LEDs are incoherent sources as compared to laser sources. For instance, LEDs may have a spectral width up to $200 \mathrm{~nm}$ while lasers exhibit a much narrower spectral width under $10 \mathrm{~nm}$. This makes it impossible to use LEDs for direct coherent beam combining in free space [5].

The coherence length of optical sources is essential to consider especially after propagating a certain distance. The various component wavelengths within the spectral range will develop different phase relationships over a frequency selective medium. The coherence length is provided as [5]:

$$
L_{c}=\frac{c}{\Delta f}
$$

where $c$ is the velocity of light and $\Delta f$ is the spectral range of a source. It is clear that an LED has a small coherence length $(\sim \mu m)$ due to its broad spectral range. Hence, it's essential to develop a method for increasing the coherence length of an LED to ensure OBS feasibility [26].

\subsubsection{LED to waveguide interface}

Since we are considering the use of SOI, it is important to keep note of the relationship between wavelength and loss. The absorption loss for silicon is high for wavelengths smaller than $1.1 \mu \mathrm{m}$. For example, the absorption of pure silicon at $\lambda=1.15 \mu \mathrm{m}$ exhibits an attenuation of $2.83 \mathrm{~dB} / \mathrm{cm}$ while a source wavelength of $\lambda=1.52 \mu \mathrm{m}$ undergoes a loss of $0.004 \mathrm{~dB} / \mathrm{cm}$ [18]. We will investigate a surface-emitting LED at $\lambda=1.55 \mu \mathrm{m}$ due to the absorption losses such as band edge absorption and free carrier absorption of silicon.

Generally, LEDs are expected to couple into a multi-mode fiber (MMF) but many 
previous experiments have shown that LEDs can launch sufficient power into a singlemode fiber $(\mathrm{SMF})$ as shown in Figure 4.3, for long distance transmission over several kilometers.
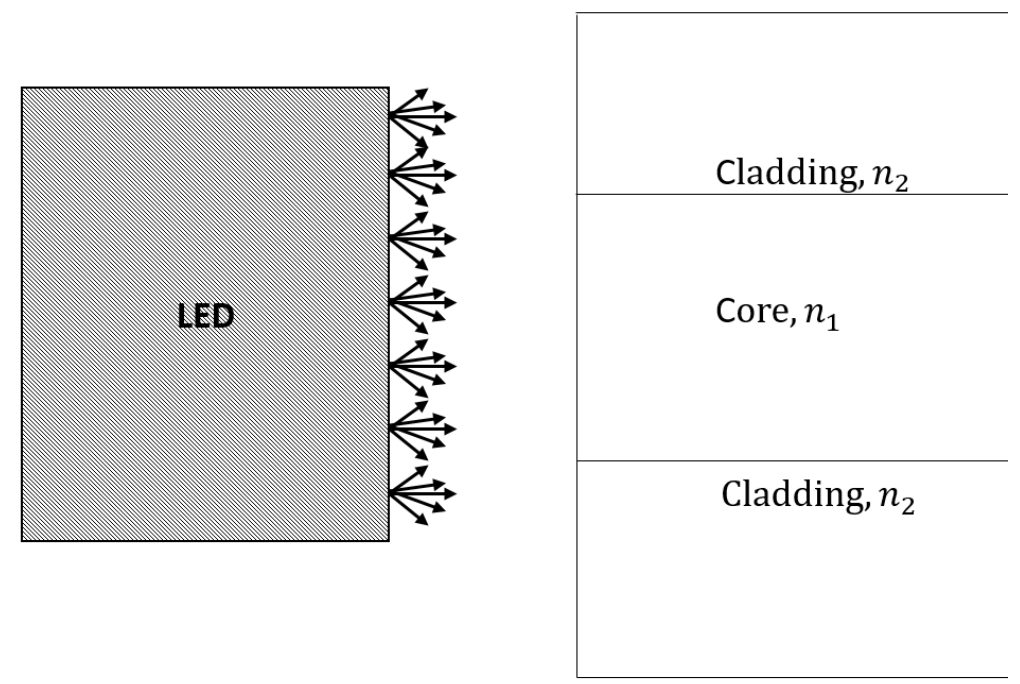

Figure 4.3: LED butt-coupling to SMF

The source radiance of the edge emitting LED is as follows 27]:

$$
B(x, y ; \phi, \theta)=\frac{d^{2} P}{d A d \omega}=B_{0} N(x, y) F(\phi, \theta)
$$

where, $B_{0}$ is the maximum brightness of the source, $N(x, y)$ and $F(\phi, \theta)$ are the spatial and angular dependence of the radiated light intensity, respectively.

Furthermore, the coupling efficiency for a butt-coupled edge emitted LED to fiber is provided as follows [27]:

$$
\begin{gathered}
\eta=T \frac{\mu+v+2}{2 \pi^{2}} \frac{\lambda_{0}^{2}}{\left[\left(2 r_{0}^{2}+L^{2}\right)\left(2 r_{0}^{2}+W^{2}\right)\right]^{1 / 2}} S_{x} S_{y} \\
S_{x}=\exp \left(-\frac{2 \alpha^{2}}{2 r_{0}^{2}+L^{2}}\right), S_{y}=\exp \left(-\frac{2 \beta^{2}}{2 r_{0}^{2}+W^{2}}\right)
\end{gathered}
$$

Let the center of the edge emitter be located at $(\alpha, \beta) . \quad \lambda_{0}$ represents the source 
wavelength. $T=4 n /(1+n)^{2}$ is the Fresnel transmission coefficient. The quantities $\mu$ and $v$ are, respectively, the transverse and lateral power distribution coefficient. $2 \mathrm{~W}$ and $2 \mathrm{~L}$ are the transverse and lateral $1 / e^{2}$ full widths of the near-field source. Finally, $r_{0}$ represents the mode field radius of the SMF.

The coupling of an LED to a monolithic waveguide circuit can be further studied, but it is assumed that an LED can be butt-coupled into a SMF then onto a SOI waveguide which is a planar waveguide consisting of $\mathrm{Si}$ and $\mathrm{SiO}_{2}$ as shown in Figure 4.4. Similar to an optical fiber, the SOI waveguide contains a cladding, which is the buried oxide layer to prevent the field from penetrating the silicon substrate.

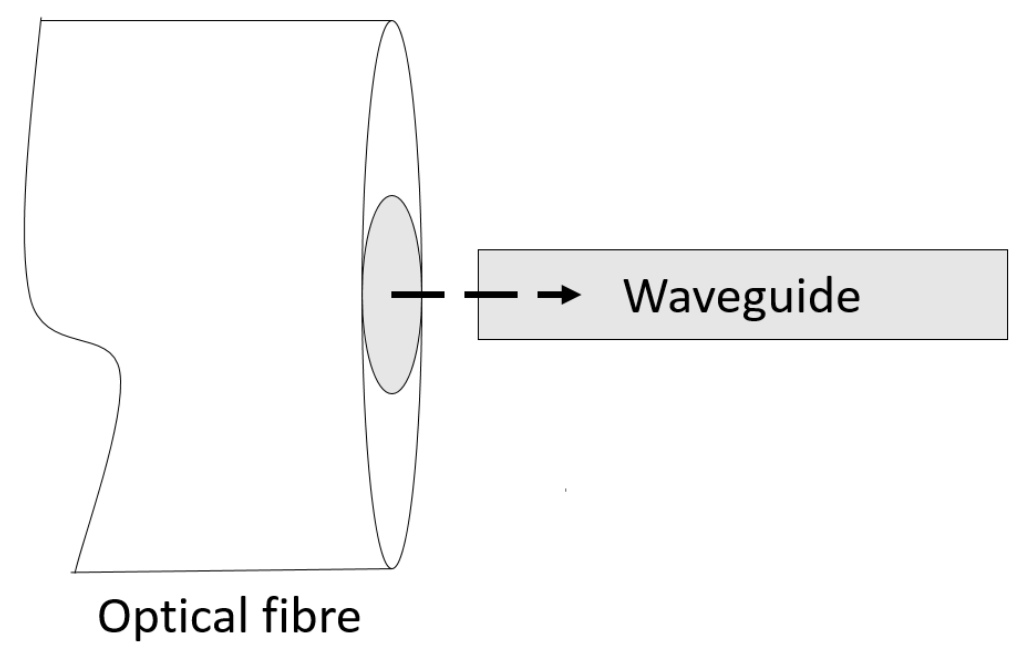

Figure 4.4: Butt-coupling from fibre to optical circuit

Generally, LDs are used for efficient coupling into a waveguide and are suitable for OPAs due to its coherence. However, LEDs are being investigated for use in an OPA. The main challenges with using an LED for a SOI waveguide, is its coupling loss and lack of coherence due to its broad spectrum. A broad spectral source will be impossible to create an effective interference pattern on the output of the OPA, hence OBS wont be achieved. In order to use a broad spectral source, there are various methods for filtering the source. Some filters which can be used is an external Piezo-Actuated Fabry-Perot Interferometer [28] or a Fiber Bragg grating with a circulator [29]. The benefit of the Fabry-Perot 
Interferometer is its tunable capability for various LED sources by adjusting the gap between the mirrors. For our purpose, we will assume that the LED source band remains relatively constant, hence a more monolithic approach will be the incorporation of ring resonators, shown in Figure 4.5. In general, a ring resonator is a looped optical waveguide which is combined with a coupling mechanism to access the loop. Ring-based resonators can be used as spectral filters which are used for multiplexing or de-multiplexing signals. Compact wavelength channel filters could also be created by means of ring resonators [30], [31]. The field which is incident in the input of the waveguide will be partly transmitted to the drop port.

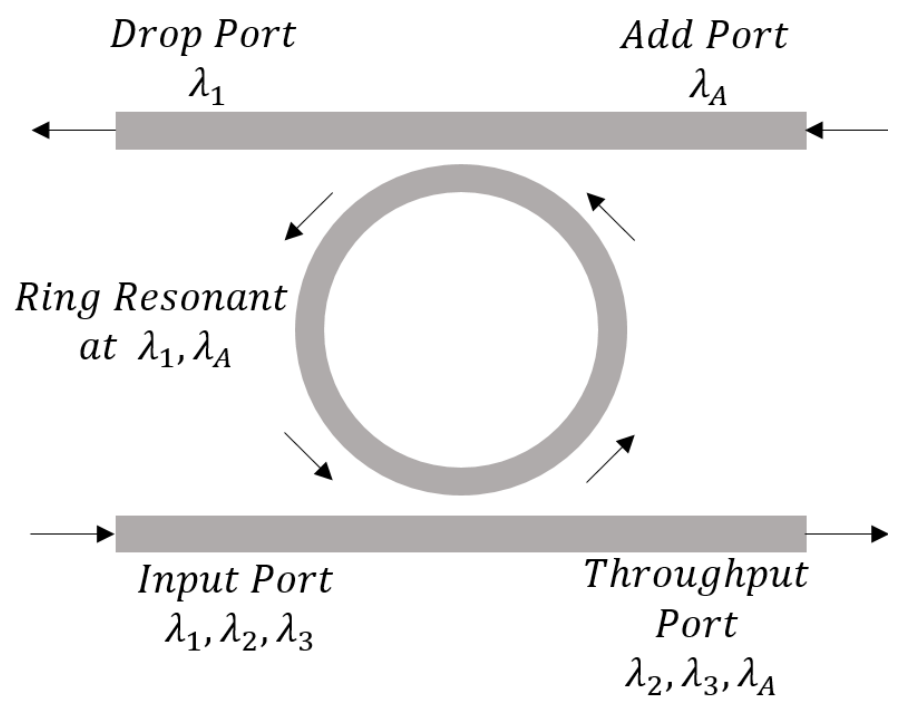

Figure 4.5: Optical ring resonator

The transmission to the pass and the drop port can also be derived. The transmission intensity is 30

$$
\begin{gathered}
T_{p}=\frac{I_{\text {pass }}}{I_{\text {input }}}=\frac{r_{2}^{2} a^{2}-2 r_{1} r_{2} a \cos (\phi)+r_{1}^{2}}{1-2 r_{1} r_{2} a \cos (\phi)+\left(r_{1} r_{2} a\right)^{2}} \\
T_{d}=\frac{I_{\text {drop }}}{I_{\text {input }}}=\frac{\left(1-r_{1}^{2}\right)\left(1-r_{2}^{2}\right) a}{1-2 r_{1} r_{2} a \cos \phi+\left(r_{1} r_{2} a\right)^{2}}
\end{gathered}
$$

where, $T_{d}$ and $T_{p}$ is the drop and pass transmission, respectively. $r$ is the self-coupling 
coefficient, $a$ is the single-pass amplitude transmission, and $\phi$ is the single-pass phase shift. This ring resonator can be integrated on SOI with the rest of the monolithic OPA in order to filter a broad spectral source required for free-space interference as shown in Figure 4.6.

Ring resonators suffice all the requirements for a functional wavelength-division multiplexing (WDM) system but it can be susceptible to fabrication and temperature fluctuations. Hence, it requires precise process control in order to fabricate a ring to resonate at a desired frequency. Hence, the use of a higher-order filter can provide a uniform pass band and overcome process variation. We will consider using a double ring resonator which mimics a wavelength drop filter. This can allow tuning on each ring which can enable resonance and shift the resonance wavelength. By tuning double rings, the ring resonators become a wavelength-selective switch [30].

After the LED has been coupled into a SOI waveguide, it is then fed through a ring resonator, an intensity modulator (IM) and finally branches out to parallel Mach-Zehnder modulators (MZMs) which will control the OBS output angle. Figure 4.6 below illustrates our overall OPA system.

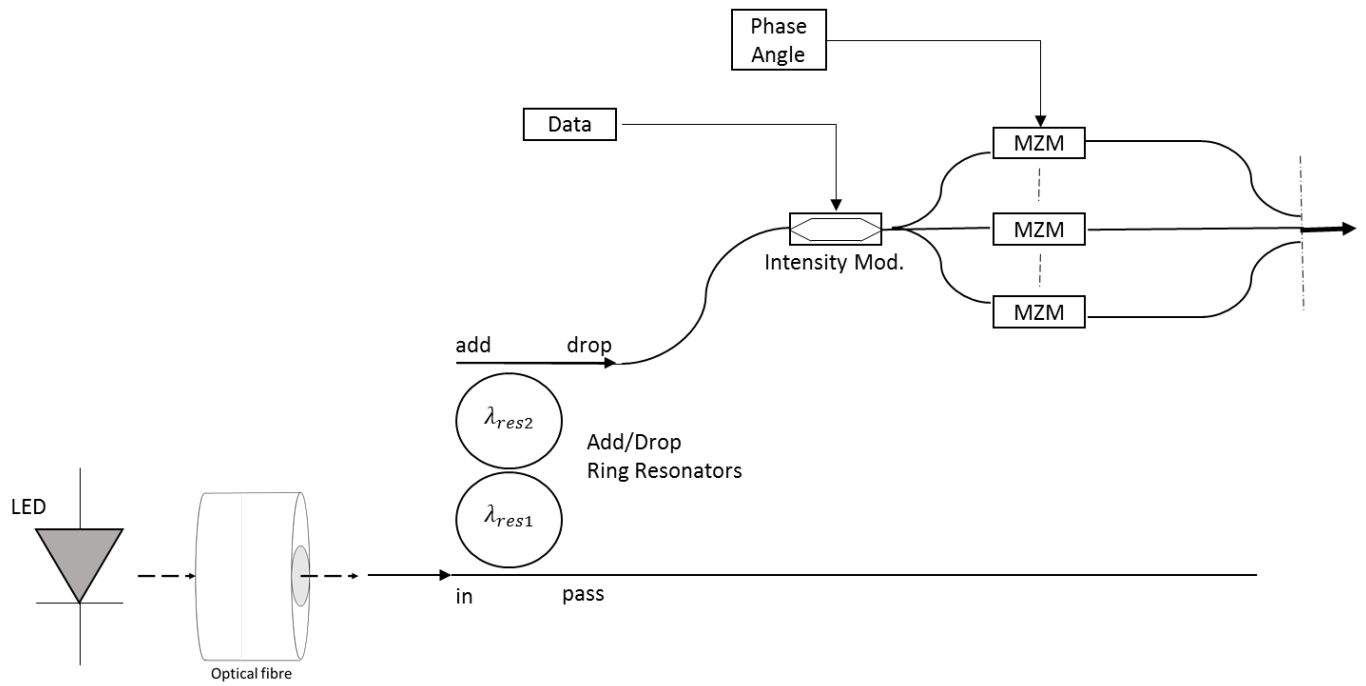

Figure 4.6: Overall SOI OPA for LED source

Another technique to filter the input LED source involves the use of a uniform fiber 
Bragg grating which reflects a specific wavelength and allows the remaining spectrum to pass [29]. The fiber Bragg grating exhibits a periodic variation of the refractive index in the core. This grating causes a band to be reflected and the remaining band to pass through. The reflected wavelength will change depending on the fiber grating period. The fiber grating period could fluctuate by temperature or strain which would change the reflected wavelength.

A circulator port is required to isolate and pass the reflection to another port as shown below in Figure 4.7. The input port is the full LED spectrum and it can be seen that the fiber Bragg grating reflects a portion of the input spectrum. Once the reflection has been isolated, it can be coupled into a SOI OPA system for OBS as previously shown.

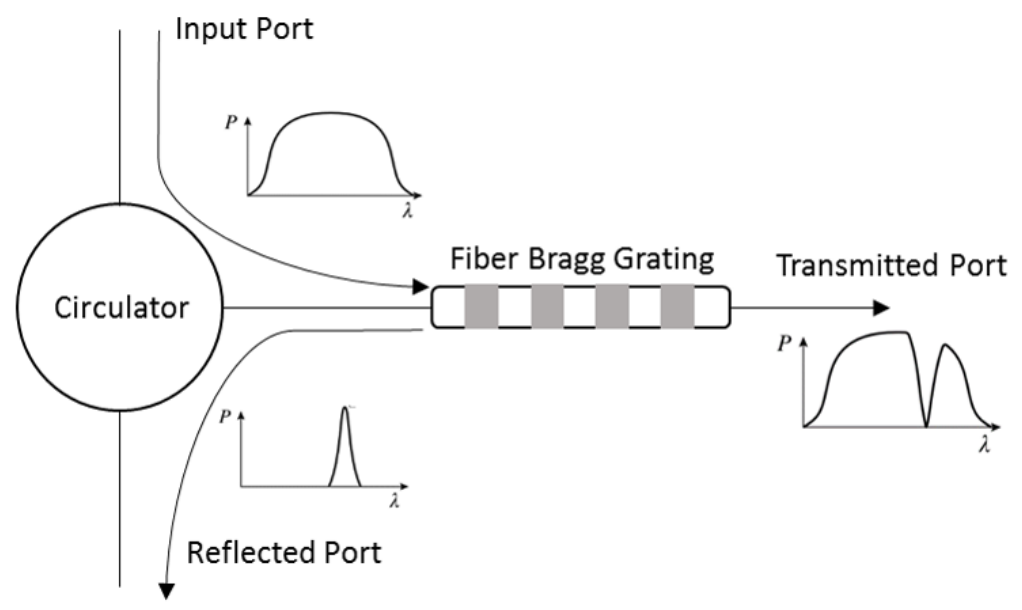

Figure 4.7: Fiber Bragg grating filter with circulator

\subsection{OPA link loss estimation}

Since the fiber and waveguide based systems are considered to output low power, in this section we provide an example link budget calculation. Table 4.1 below summarizes the estimated power and attenuation values at various points of the proposed system, assuming a high sensitivity avalanche PD (APD) is being used as the receiver. Although the LED output across the spectrum can be approximated to be around $18 \mathrm{dBm}$, the 
ring resonator quality factor determines the remaining power in the drop port. In this case, we will consider that the remaining spectrum power is around $0.9 \mathrm{dBm}$. The loss parameters are obtained from [8], 32] and, [33].

Table 4.1: LED-OPA link budget

\begin{tabular}{lcc}
\hline Component & Output/Loss & Power margin(dB) \\
\hline LED drop port power & $0.9 \mathrm{dBm}$ & \\
APD sensitivity & $-32 \mathrm{dBm}$ & \\
Acceptable loss & & 32.9 \\
LED to SMF coupler & $-11 \mathrm{~dB}$ & 21.9 \\
SMF to Waveguide OPA & $-4 \mathrm{~dB}$ & 17.9 \\
Ring Resonator dropping filter & $-0.2 \mathrm{~dB}$ & 17.7 \\
Intensity modulator & $>-1 \mathrm{~dB}$ & 16.7 \\
MZM array & $<-12 \mathrm{~dB}$ & 4.7 \\
Overall waveguide length loss & $>-1.5 \mathrm{~dB}$ & 3.2 \\
\hline
\end{tabular}

It is clear that from our link budget calculation in Table 4.1, there will be an allowable loss of $\approx 3.2 \mathrm{~dB}$ at the output of the waveguide system which is suitable for short distance OWC. According to Table 2.1, this is sufficient for a FSO link of around $300 \mathrm{~m}$. However, there have been techniques to dramatically increase the total LED power [8]. For the remainder of this thesis, we will focus on the use of a continuous wave (CW) laser source. 


\section{Chapter 5}

\section{Optical Beam Steering Simulations}

\subsection{OptiSystem ${ }^{\mathrm{TM}}$}

In this Chapter, we will first begin by demonstrating the far-field radiation of a single waveguide element in free-space. The single waveguide will consist of a core and a cladding. We will shift to a monolithic OPA by initially demonstrating a fiber array system which requires a complex optimization of lenses and array spacing to create a functional OBS. Hence, instead of using an array of fibers, an SOI OPA can achieve an array of waveguides which are spaced much closer, satisfying an essential requirement of OBS.

Once the OPA is designed, OptiSystem ${ }^{\mathrm{TM}}$ can integrate a waveguide designed using $\mathrm{OptiBPM}^{\mathrm{TM}}$ by importing an equivalent S-parameters matrix of the waveguide into the system as shown in Figure 5.1. This can be done for further investigation on the performance of the overall electro-optic system. For now, OptiSystem ${ }^{\mathrm{TM}}$ can only plot the envelope of the phase modulation. 


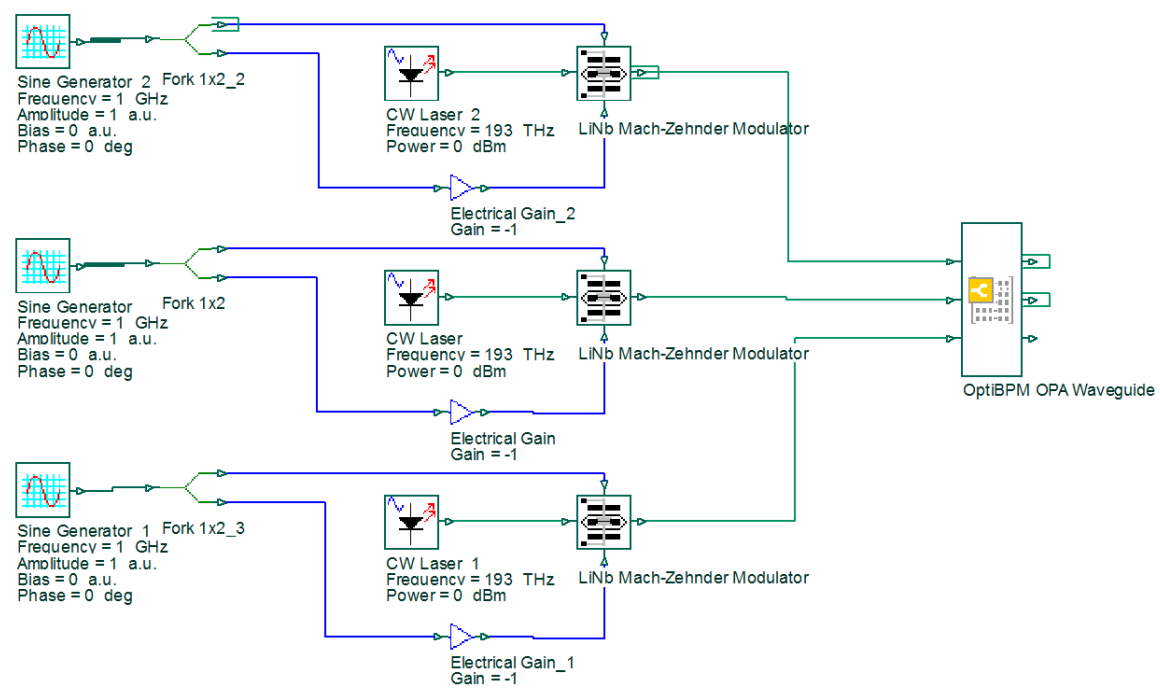

Figure 5.1: OptiSystem ${ }^{\mathrm{TM}}$ schematic with integrated OptiBPM ${ }^{\mathrm{TM}}$ waveguide

Figure 5.2 illustrates the spectrum which was measured at the input and output ports in OptiSystem ${ }^{\mathrm{TM}}$. This spectrum of a CW laser source was used to measure the output power of the OPA waveguide. The minimization of cross-coupling was also measured in OptiSystem $^{\mathrm{TM}}$ by importing the S-parameters into OptiBPM ${ }^{\mathrm{TM}}$.

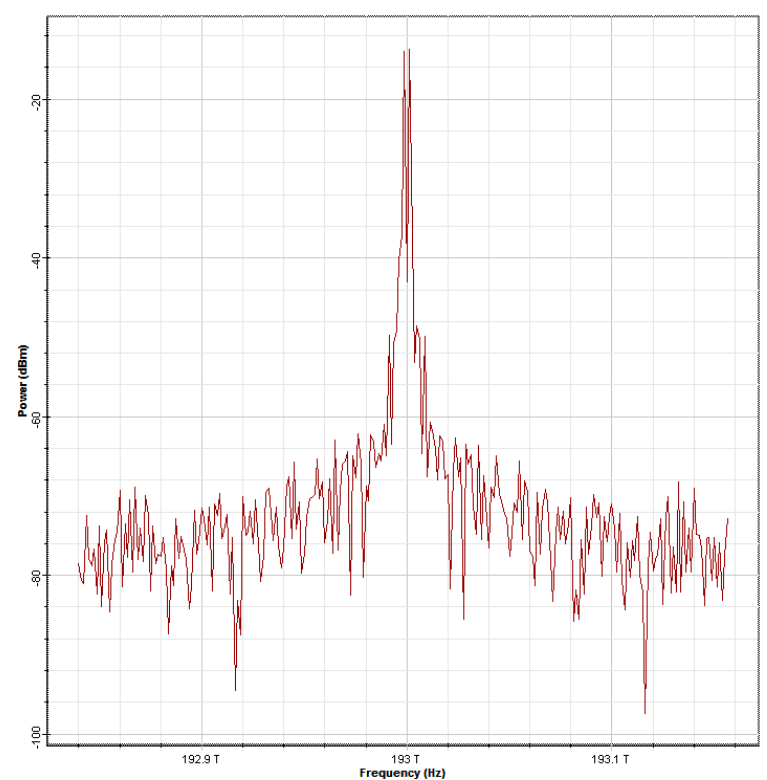

Figure 5.2: OptiSystem ${ }^{\mathrm{TM}}$ spectrum analyzer of output 


\subsection{Single waveguide}

In order to observe the feasibility of a monolithic OBS implementing an OPA, it is essential to simulate the EM fields using a finite-difference time-domain (FDTD) algorithm which divides space and time into a grid to simulate Maxwells equations. The following section will include simulations from MEEP ${ }^{\mathrm{TM}}$. $\mathrm{MEEP}^{\mathrm{TM}}$ is a versatile unitless software that provides the freedom for designing various materials, which is especially advantageous in our application to simulate the interaction between the SOI waveguide and free-space. We will assume the coupling interface is designed such that to ensure singlemode propagation. A perfectly matched layer is placed at the outer boundaries so as to prevent any reflections which will disrupt the coherent combining in free-space.

We will first begin by demonstrating the far-field radiation of a single waveguide element in free-space. This is important to ensure that enough power radiates after propagating through the waveguide. It can be shown in Figure 5.3a below that a single CW laser source guided in a waveguide element produces a broad radiation. From 0 to $12 \mu \mathrm{m}$, the field is propagating through silicon, which has a refractive index of 3.44 and incident on free space which has a refractive index of approximately 1.

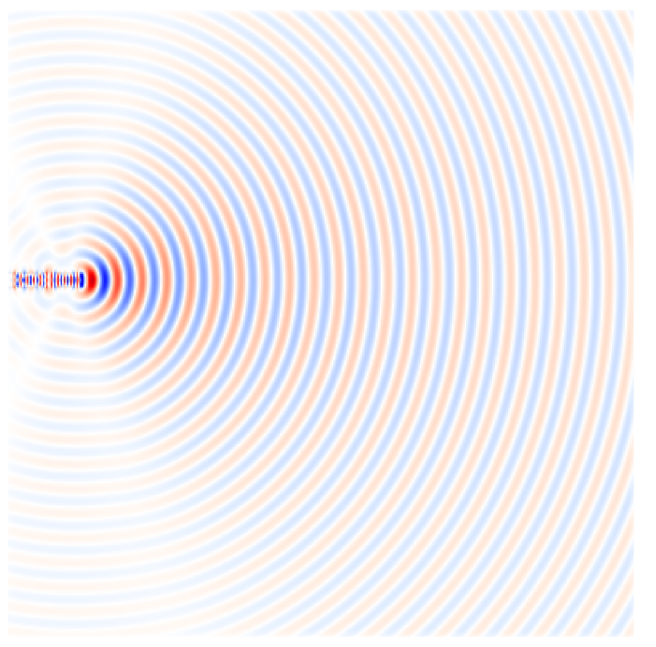

(a) FDTD simulation

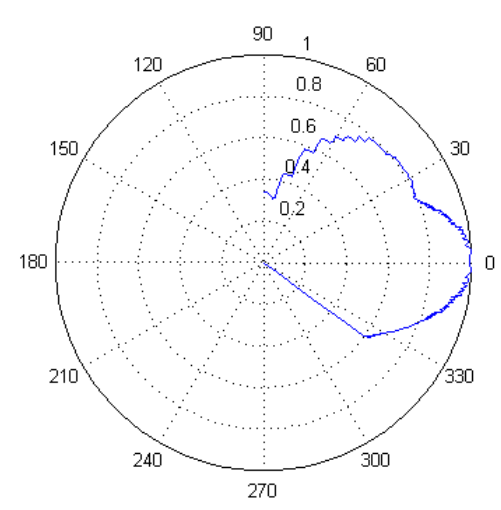

(b) Polar plot of radiation

Figure 5.3: Polar and EM plot of single element in the far-field 
In order to be in the far-field (Fraunhofer) region, the following conditions must be met [34]:

$$
\begin{aligned}
& R>\frac{2 D^{2}}{\lambda} \\
& R>>D \\
& R>>\lambda
\end{aligned}
$$

where $\mathrm{D}$ is the maximum linear dimension of the elements. It is clear that Figure $5.3 \mathrm{a}$ includes the Fraunhofer region. Figure $5.3 \mathrm{~b}$ is the polar equivalent of the magnitude of the far-field region of Figure 5.3a. We can see that the intensity, and hence the power is dispersed with a significant half-power beam width (HPBW) and low directivity. It is our goal to narrow the beam width of the far-field radiation, as stated earlier in order to illustrate functional beam forming.

\subsection{Fiber based OBS}

Since we will adopt conventional phased array techniques used in RF, we will begin by simulating an array of fibers to create a OPA. Figure 5.4 illustrates the FDTD simulation of a fiber array.

It is clear from Figure 5.4 that the output at the Fraunhofer region is producing "dead" pockets of no radiation. The reason for this effect is that the fiber consists of a core and cladding, when combined in an array, is far greater than the vacuum wavelength of $1.55 \mu \mathrm{m}$. In order to overcome this issue, a lens based system may be implemented 35 .

In a lens based FSO terminal, the uniform optical beam becomes an Airy distribution once focused with a lens after collimation [36]. For a linear FSO OPA, the far-field pattern along the direction of $\theta$ with respect to the Airy distribution at each element, $n$ 


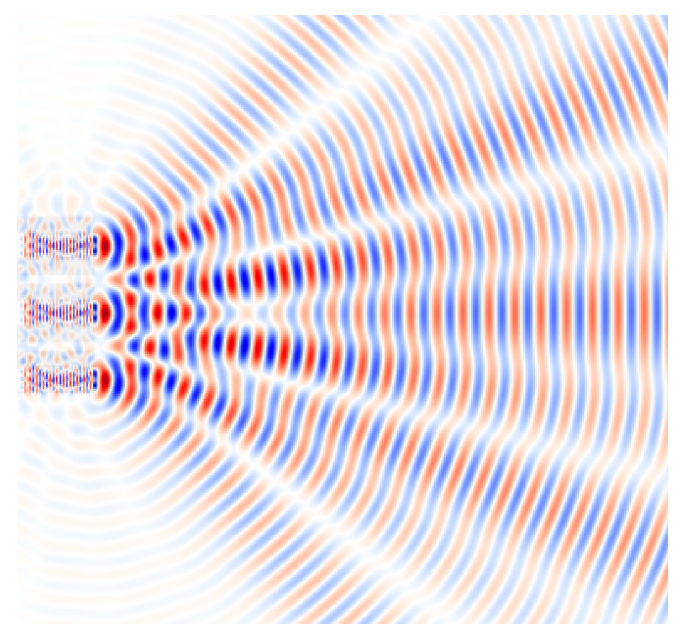

Figure 5.4: Far-field radiation of fiber array implementation

can be expressed as

$$
E(\theta, t)=\sum_{n=1}^{N} \frac{\pi D^{2}}{4 \lambda f}\left[\frac{2 J_{1}(k D r / 2 f)}{k D r / 2 f}\right] \exp \left(j \omega_{m} t\right) * \exp \left[j\left(-n k_{m} d \sin (\theta)+n\left(\frac{\omega_{m}}{c}\right) d \sin (\theta)\right)\right]
$$

where $D, f, k, \lambda, r$, and $J_{1}$ is the lens diameter, focal length, wavenumber, optical wavelength, radial distance, and the first order Bessel function, respectively. $\omega_{m}$ is the microwave frequency, $k_{m}=\omega_{m} / c$ is the wave vector, and $d$ is the distance between radiating elements approximated to be on the order of microns. Figure 5.5 illustrates a potential setup which combines the use of a fiber, microlens array, and true-time delay as the OPA to create interference.

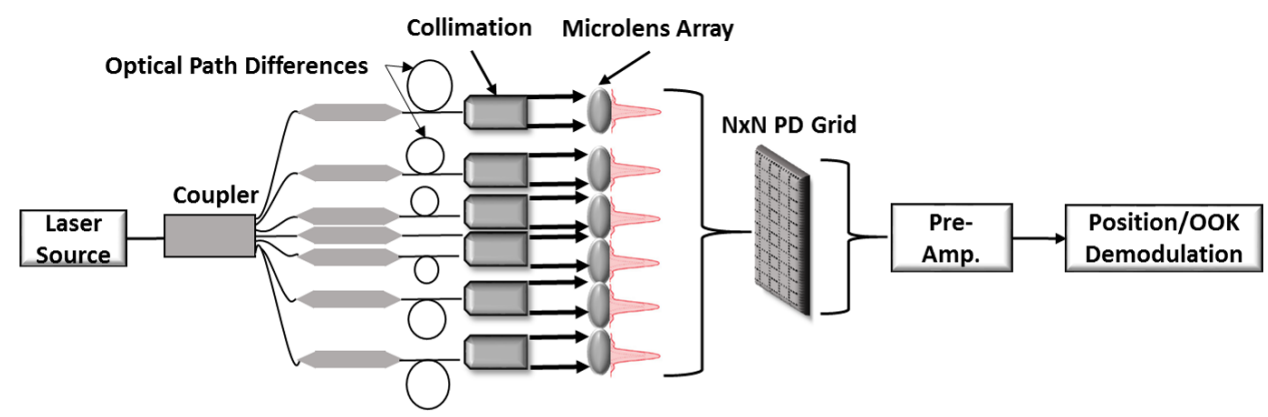

Figure 5.5: A lens based FSO terminal 


\subsection{SOI OBS with OPA}

With the advantage of SOI as previously discussed, a waveguide grating can be fabricated. We can obtain OBS by spacing the waveguides within the infrared wavelength of $1.55 \mu \mathrm{m}$. We will begin by demonstrating the range for phase shift in silicon using OptiFDTD ${ }^{\mathrm{TM}}$. Then the waveguide OPA dimensions will be referenced using OptiBPM ${ }^{\mathrm{TM}}$. The spacing and thickness of the waveguide will be simulated to ensure single mode propagation within the waveguide and that there is no internal cross-talk prior to free-space propagation. Finally, the dimensions to design the OPA will be varied in MEEP ${ }^{\mathrm{TM}}$ to illustrate the OBS functionality.

\subsubsection{Optiwave}

A 3D illustration of the slab waveguide can be shown using OptiFDTD ${ }^{\mathrm{TM}}$ as shown in Figure 5.6 a below. To illustrate the refractive index accuracy on the phase shift, Figure $5.6 \mathrm{~b}$ below illustrates a time domain (femtosecond) representation of the propagating electric field. In this case, a $180^{\circ}$ phase shift is shown.

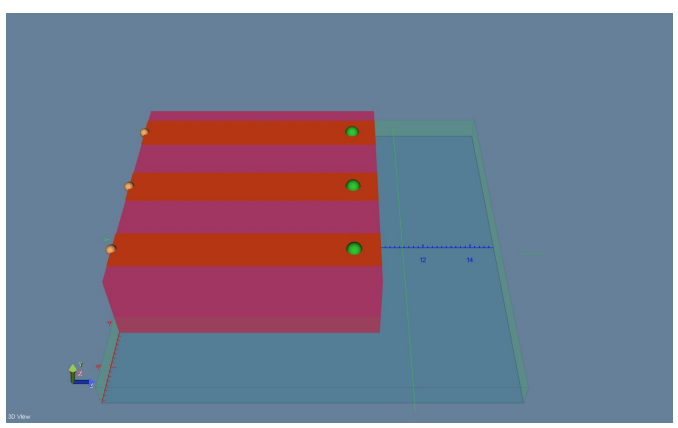

(a) 3D slab waveguide

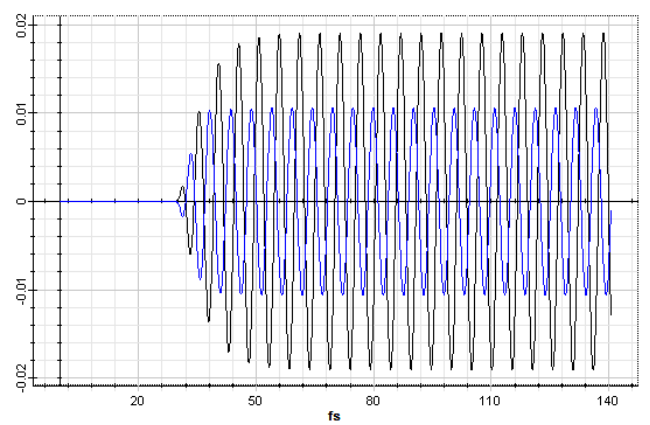

(b) Phase shift in optical time domain

Figure 5.6: $0.95 \mu \mathrm{m}$ element spacing with $\beta_{1}=55^{\circ}$ and $\beta_{2}=74^{\circ}$ configuration

A scattering script was created to analyze the FDTD result of the waveguide grating where each grating has a width of $1.6 \mu \mathrm{m}$ and spacing of $0.95 \mu \mathrm{m}$ in OptiBPM ${ }^{\mathrm{TM}}$ as shown in Figure 5.7. It can clearly be observed that single mode propagation is achieved with the implemented dimensions. 


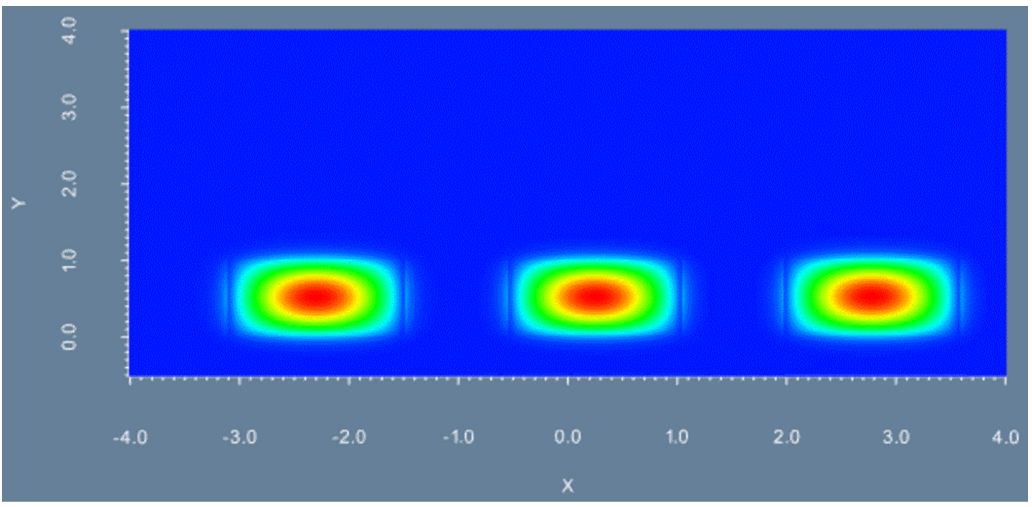

Figure 5.7: OptiBPM ${ }^{\mathrm{TM}}$ scattering matrix simulation

The following matrices illustrate the simulated scattering (S)-parameters for the 3port waveguide, exported from OptiBPM ${ }^{\mathrm{TM}}$. It is important to note that $S_{11}, S_{22}$, and $S_{33}$ are the gain from the input to the output and not the reflection. At $\lambda=1.51 \mu \mathrm{m}$ :

$$
\left(\begin{array}{ccc}
1 \angle-1.03 & 9.99 \times 10^{-6} \angle 0.0601 & 9.99 \times 10^{-6} \angle 0.22 \\
9.99 \times 10^{-6} \angle 0.0601 & 1 \angle-1.03 & 9.99 x 10^{-6} \angle 0.0604 \\
9.99 \times 10^{-6} \angle 0.2157 & 9.99 x 10^{-6} \angle 0.0677 & 0.99 \angle-1.0276
\end{array}\right)
$$

Furthermore, the S-parameters were simulated with lower frequency. At $\lambda=1.61 \mu \mathrm{m}$ :

$$
\left(\begin{array}{ccc}
1 \angle-0.965 & 9.99 \times 10^{-6} \angle 0.229 & 9.99 \times 10^{-6} \angle 0.825 \\
9.99 \times 10^{-6} \angle 0.2291 & 1 \angle-0.9646 & 9.99 \times 10^{-6} \angle 0.2296 \\
9.99 \times 10^{-6} \angle 0.826 & 9.99 \times 10^{-6} \angle 0.231 & 0.99 \angle-0.965
\end{array}\right)
$$

From the variation of wavelength, we can see that the gain is almost unity with very low loss from the input to output ports, primarily due to a small waveguide length of $10 \mu \mathrm{m}$. It is also clear that there is minimal internal cross coupling among the ports which is essential for free-space coherent combining at the output.

\subsubsection{Free-space OBS simulations}

In this section, OBS will be illustrated in $\mathrm{MEEP}^{\mathrm{TM}}$ using the OPA waveguide dimensions from OptiBPM ${ }^{\mathrm{TM}}$ as a reference. It was determined from OptiBPM ${ }^{\mathrm{TM}}$ that a waveguide 
width of $1.6 \mu \mathrm{m}$ and spacing of $0.95 \mu \mathrm{m}$ ensures single mode propagation and no crosscoupling. Hence, this provides a reference point for sweeping the waveguide spacing and relative phase shifts. It is important to note that in these MEEP ${ }^{\mathrm{TM}}$ simulations, we are designing the passive component of the OPA. It is assumed that the EOM can achieve the required shift in refractive index to produce an optical phase shift in each waveguide. Figure 5.8a below illustrates our simulation of a silicon waveguide array with $0.62 \mu \mathrm{m}$ spacing between each element, a waveguide width of $0.93 \mu \mathrm{m}$, a vacuum wavelength of $1.5 \mu \mathrm{m}$, and a phase shift to steer the beam in a specific angle.

In the following figures, we will observe the variation in HPBW, and directivity as the spacing, relative phase shift, and when the vacuum wavelength varies from $1538 \mathrm{~nm}$ to $1612 \mathrm{~nm} . \beta_{1}$ is the relative phase shift between the primary waveguide and the secondary waveguide, $\beta_{2}$ is the relative phase shift between the secondary and tertiary waveguide. We will observe the beam forming by the polar plot as shown in Figure 5.8b.

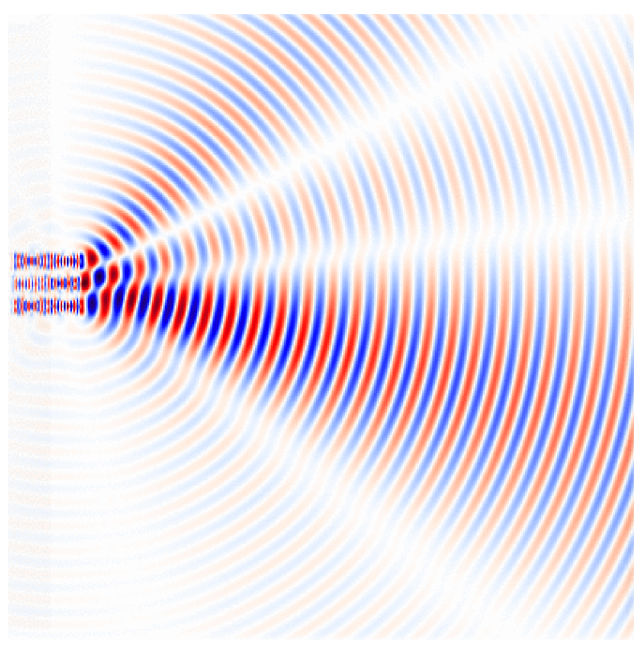

(a) FDTD simulation

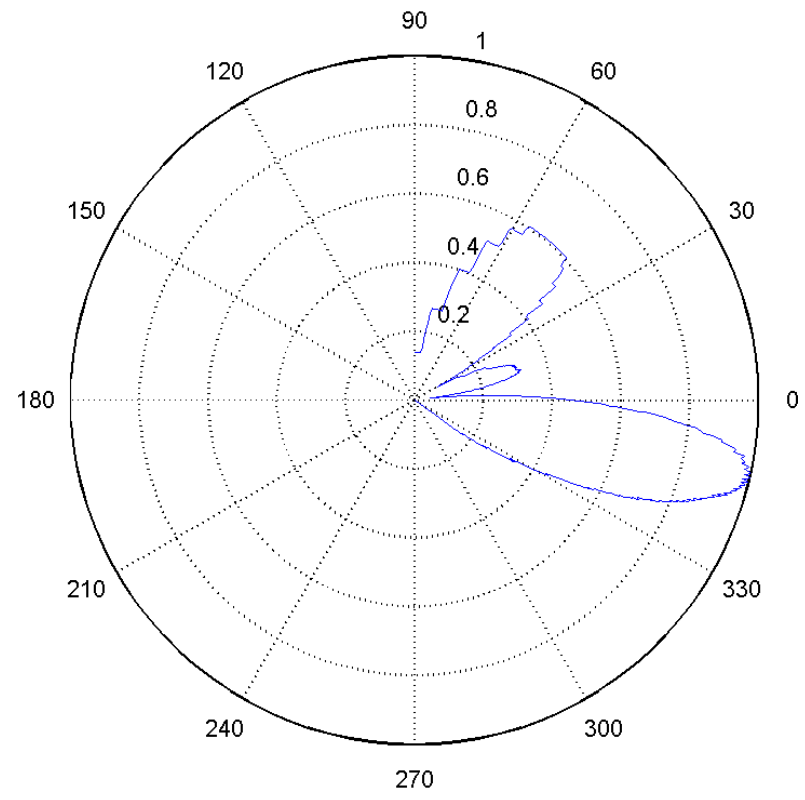

(b) Polar plot of radiation

Figure 5.8: $0.62 \mu \mathrm{m}$ element spacing with $\beta_{1}=55^{\circ}$ and $\beta_{2}=74^{\circ}$ configuration 
From Figure 5.8 we can see the significant increase in directivity as compared to Figure 5.3. From this figure, we can clearly observe a main-lobe and multiple side-lobes. Although, it is not possible to fully eliminate the side lobes, it is desired to configure the setup so as to minimize the power radiated from the side lobes and increase the main lobe power. Further optimization of phase, spacing, and waveguide dimensions can be done in order to increase the directivity.

Additionally, it is important to note the frequency variation with the beam forming output. We can see that from Figure 5.9a 5.10b, the side-lobe magnitude increases as the element spacing increases, regardless of the slight variation in frequency. With all cases, the largest wavelength, $1612 \mathrm{~nm}$ decreases the magnitude of the side lobes.

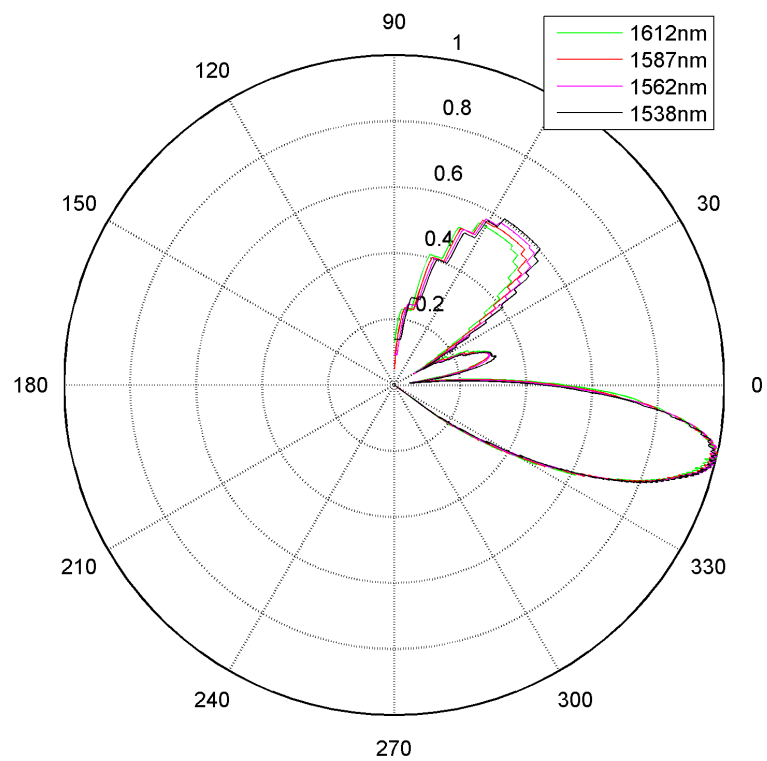

(a) $0.62 \mu \mathrm{m}$ element spacing

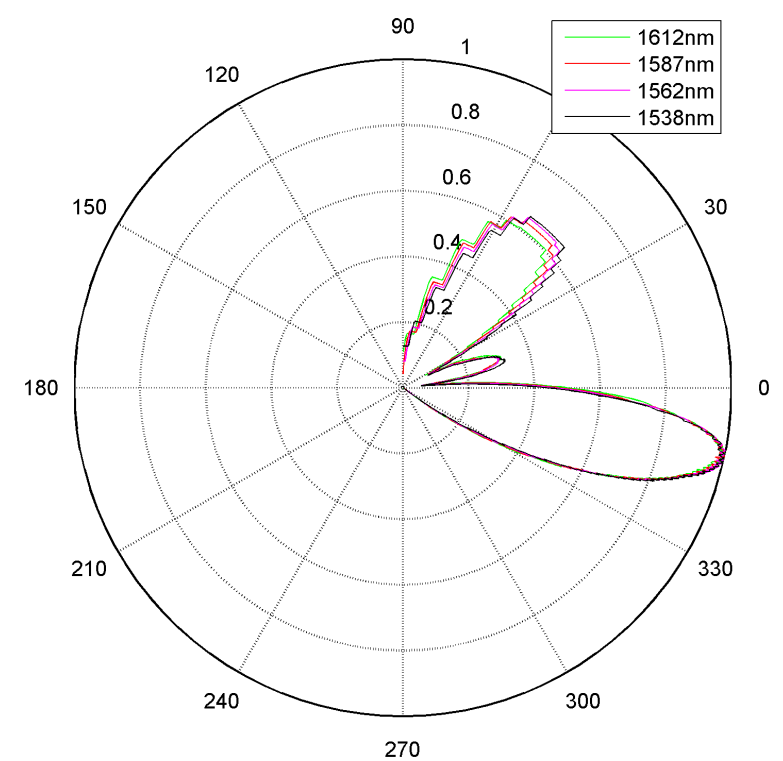

(b) $0.78 \mu \mathrm{m}$ element spacing

Figure 5.9: Frequency variation of $0.62 \mu \mathrm{m}$ and $0.78 \mu \mathrm{m}$ element spacing with $\beta_{1}=55^{\circ}$ and $\beta_{2}=74^{\circ}$ configuration 


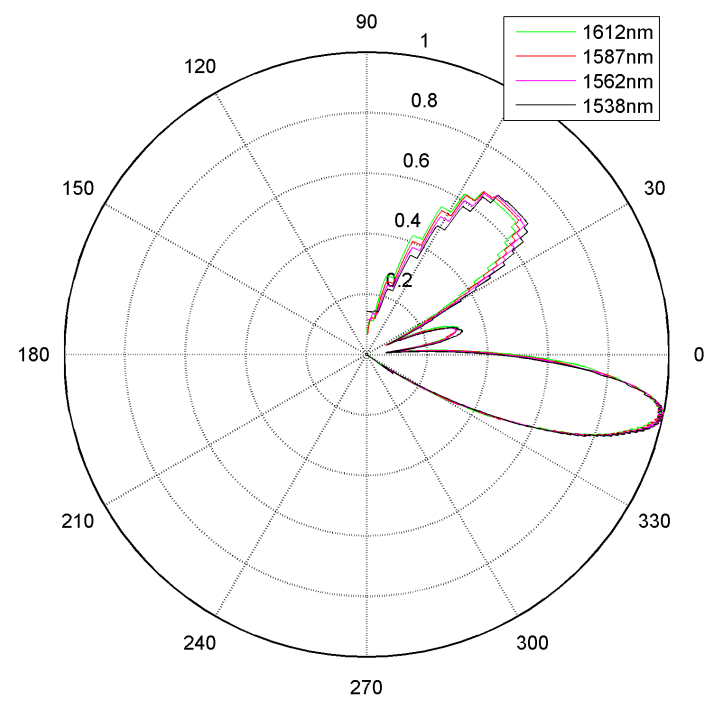

(a) $0.93 \mu m$ element spacing

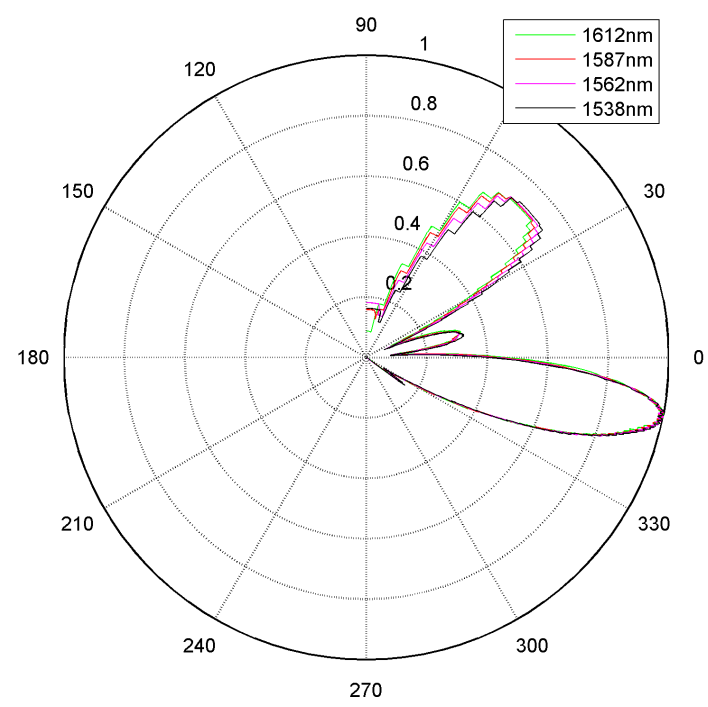

(b) $1.25 \mu \mathrm{m}$ element spacing

Figure 5.10: Frequency variation of $0.93 \mu \mathrm{m}$ and $1.25 \mu \mathrm{m}$ element spacing with $\beta_{1}=55^{\circ}$ and $\beta_{2}=74^{\circ}$ configuration

Figure 5.11a 5.12billustrates a cross section of the polar beam angle. With the current configurations, the simulations show a steering capable of $45^{\circ}$. The increase in spacing from $0.62 \mu \mathrm{m}$ to $1.25 \mu \mathrm{m}$ significantly increases the magnitude of the side lobes, and decreases the overall steering range.

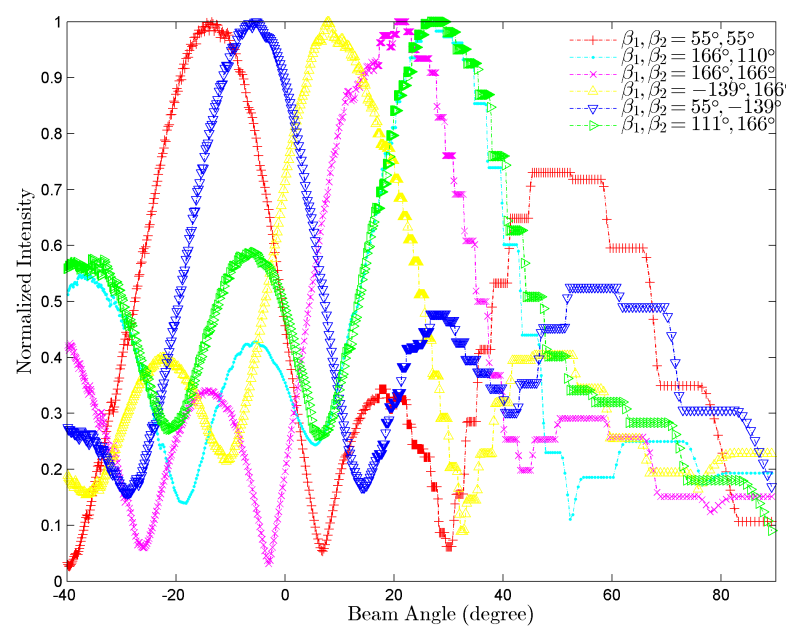

(a) $0.62 \mu m$ element spacing

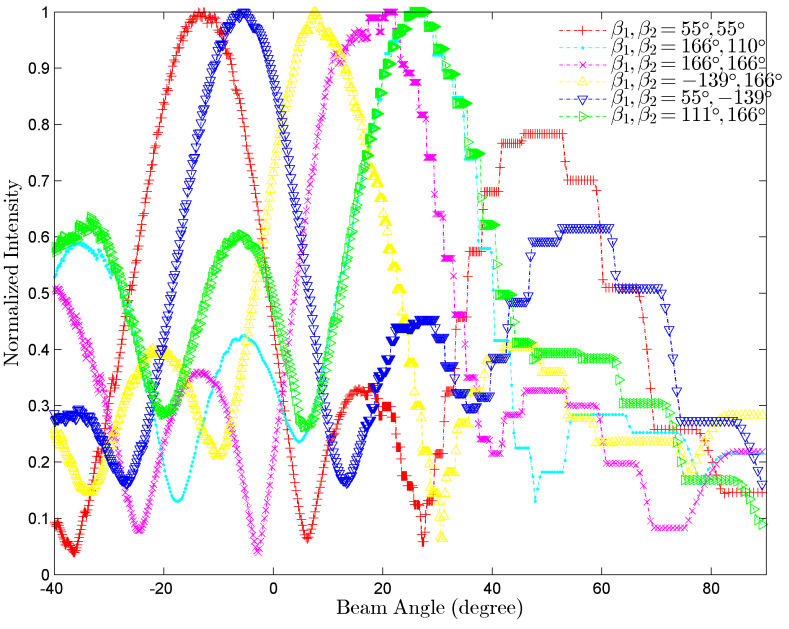

(b) $0.78 \mu m$ element spacing

Figure 5.11: Beam angle variation for $0.62 \mu \mathrm{m}$ and $0.78 \mu \mathrm{m}$ element spacing 


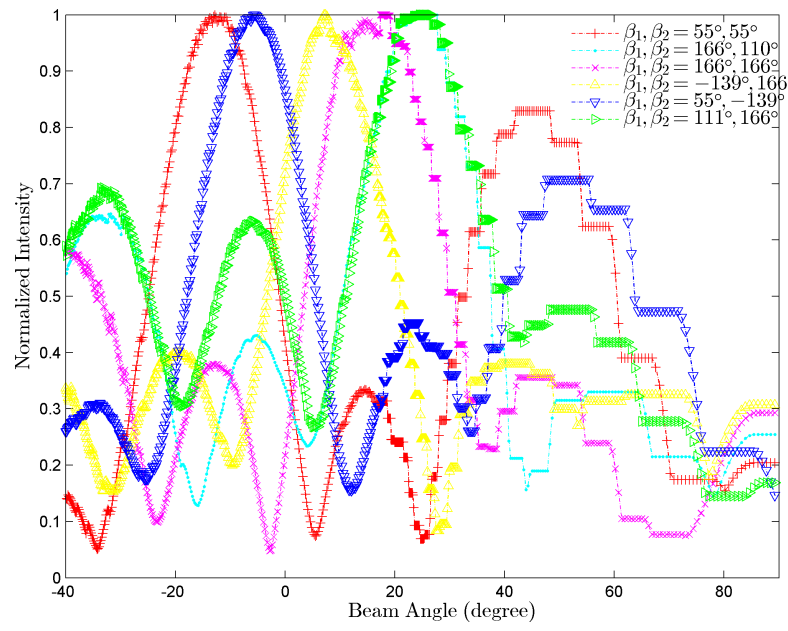

(a) $0.93 \mu \mathrm{m}$ element spacing

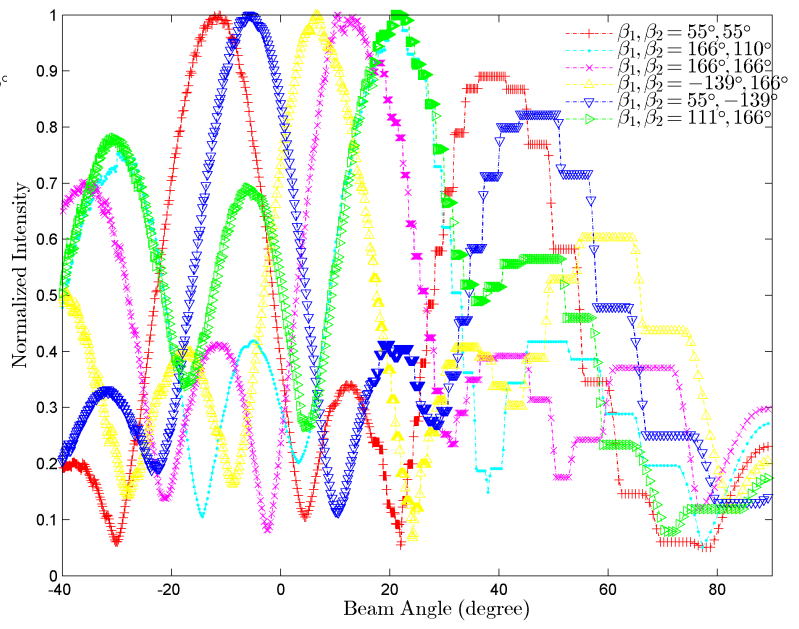

(b) $1.25 \mu \mathrm{m}$ element spacing

Figure 5.12: Beam angle variation for $0.93 \mu \mathrm{m}$ and $1.25 \mu \mathrm{m}$ element spacing

\subsubsection{HPBW and Directivity}

In accordance with Figure 5.9a $5.10 \mathrm{~b}$, the HPBW in Figure 5.13 and the directivity in Figure $5.14 \mathrm{a}$ illustrate an overall decrease along an increase in waveguide spacing.

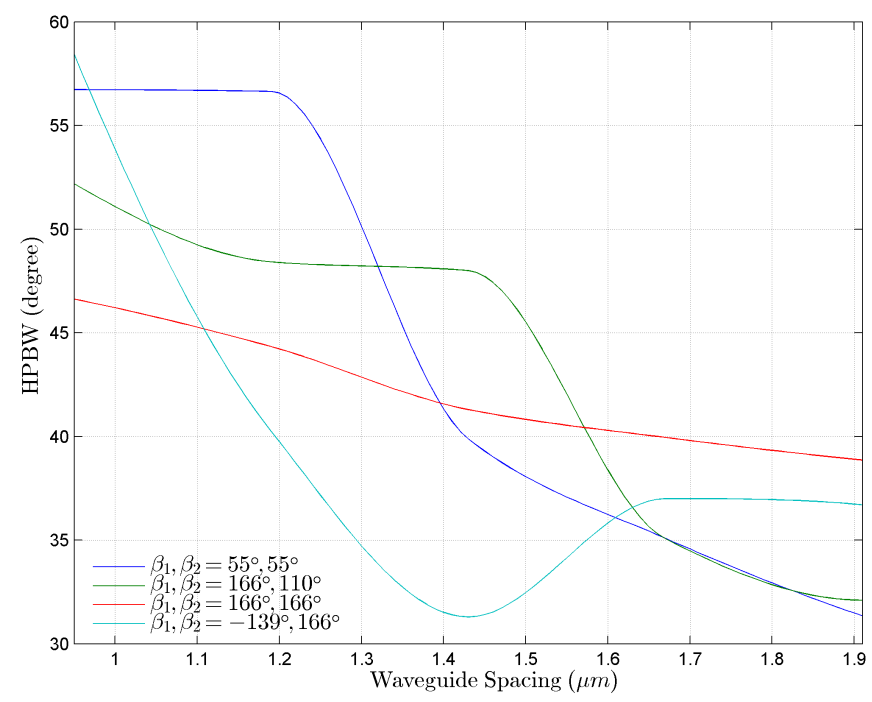

Figure 5.13: HPBW variation with increase in element spacing

Figure 5.13 and Figure 5.14a illustrates the HPBW and Directivity, respectively. We also investigated the use of a Gaussian beam instead of a CW laser source as shown in 
Figure 5.14a. Gaussian beams occur with a divergence in the laser beam, inducing a waist in the beam intensity profile. There is a non-monotonic behaviour in directivity as the waveguide spacing increases, which illustrates an opposite trend from Figure 5.14b,

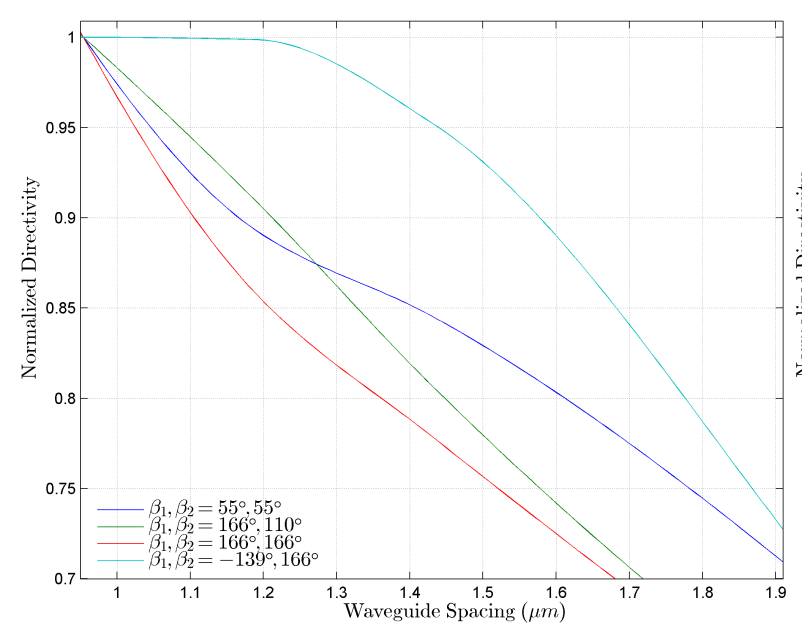

(a) Directivity of CW source

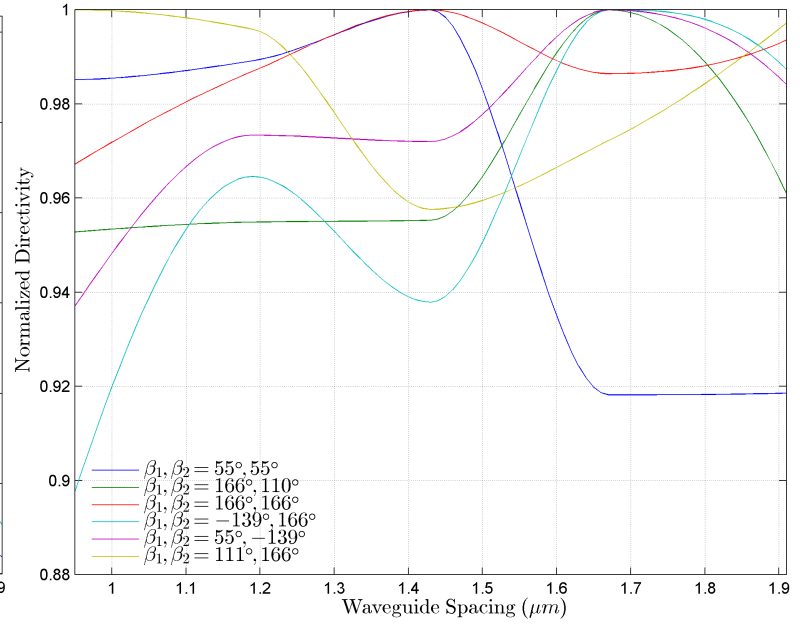

(b) Directivity of Gaussian beam with waist size, $71 \mathrm{~nm}$

Figure 5.14: Directivity variation with increase in element spacing

Figure 5.15 illustrates our FDTD simulation for the OPA with feedback which was previously introduced in Figure 4.2. There are two couplers stemming from each branch which is terminated to a matched layer. In practice, these matched layers would be replaced by a balanced PD to sense the amplitude or phase of the interference.

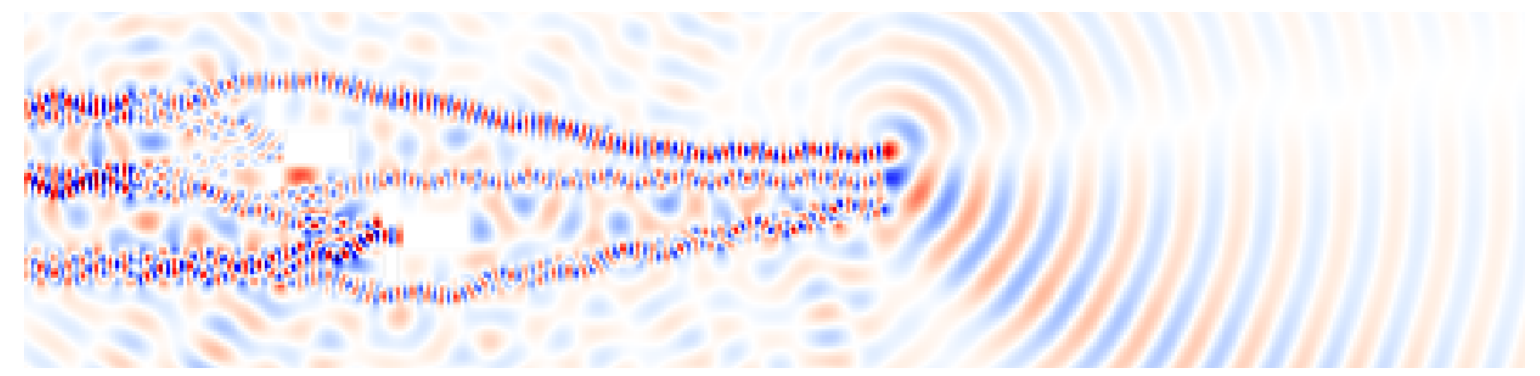

Figure 5.15: Far-field radiation of feedback configuration 
Table 5.1 5.4 illustrates the number of side-lobes, SNR (dB) and the main-lobe/sidelobe peak to peak intensity ratio $(\mathrm{dB})$ for the worst case scenario for each relative phase shift. To calculate the SNR, the mean-square shot noise current is $\left\langle i_{\text {shot }}^{2}\right\rangle=8.68 \times 10^{-14} A^{2}$ and the mean-square thermal noise current for the receiver is $\left\langle i_{T}^{2}\right\rangle=3.23 x 10^{-16} A^{2}$. We will not take into consideration the effect of dark current. For reference, the radiation of a single waveguide with one source and no beam forming is listed in the first row of each Table.

The relative phase shifts which are most sensitive to process variation along the waveguide spacing is when a relative phase shift of $\beta_{1}$ and $\beta_{2}$ equals $166^{\circ}$ and $110^{\circ}$, respectively. The relative phase shifts which provide the most stable main-lobe to side-lobe radiation power is when $\beta_{1}$ and $\beta_{2}$ are equal to each other as well as when $\beta_{1}$ and $\beta_{2}$ equals $-139^{\circ}$ and $166^{\circ}$, respectively.

Table 5.1: Optical beam forming radiation with an element spacing of $0.62 \mu \mathrm{m}$

\begin{tabular}{lcccc}
\hline$\beta_{1}$ & $\beta_{2}$ & Number of side-lobes & Main-lobe/Side-lobe intensity (dB) & SNR (dB) \\
\hline N.A. & N.A. & 0 & N.A. & 23.57 \\
$55^{\circ}$ & $55^{\circ}$ & 2 & 2.79 & 33.24 \\
$166^{\circ}$ & $110^{\circ}$ & 3 & 5.15 & 34.42 \\
$-139^{\circ}$ & $166^{\circ}$ & 2 & 7.96 & 35.83 \\
$55^{\circ}$ & $-139^{\circ}$ & 3 & 6.02 & 34.86 \\
$111^{\circ}$ & $166^{\circ}$ & 2 & 4.45 & 34.07 \\
\hline
\end{tabular}

Table 5.2: Optical beam forming radiation with an element spacing of $0.78 \mu \mathrm{m}$

\begin{tabular}{lcccc}
\hline$\beta_{1}$ & $\beta_{2}$ & Number of side-lobes & Main-lobe/Side-lobe intensity $(\mathrm{dB})$ & SNR $(\mathrm{dB})$ \\
\hline N.A. & N.A. & 0 & N.A. & 23.57 \\
$55^{\circ}$ & $55^{\circ}$ & 2 & 2.14 & 32.92 \\
$166^{\circ}$ & $110^{\circ}$ & 3 & 4.55 & 34.12 \\
$-139^{\circ}$ & $166^{\circ}$ & 3 & 7.90 & 35.80 \\
$55^{\circ}$ & $-139^{\circ}$ & 3 & 4.15 & 33.92 \\
$111^{\circ}$ & $166^{\circ}$ & 2 & 4.14 & 33.92 \\
\hline
\end{tabular}


Table 5.3: Optical beam forming radiation with an element spacing of $0.93 \mu \mathrm{m}$

\begin{tabular}{lcccc}
\hline$\beta_{1}$ & $\beta_{2}$ & Number of side-lobes & Main-lobe/Side-lobe intensity (dB) & SNR (dB) \\
\hline N.A. & N.A. & 0 & N.A. & 23.57 \\
$55^{\circ}$ & $55^{\circ}$ & 2 & 1.72 & 32.71 \\
$166^{\circ}$ & $110^{\circ}$ & 4 & 3.97 & 33.83 \\
$-139^{\circ}$ & $166^{\circ}$ & 3 & 7.88 & 35.79 \\
$55^{\circ}$ & $-139^{\circ}$ & 3 & 3.09 & 33.39 \\
$111^{\circ}$ & $166^{\circ}$ & 3 & 3.11 & 33.40 \\
\hline
\end{tabular}

Table 5.4: Optical beam forming radiation with an element spacing of $1.25 \mu \mathrm{m}$

\begin{tabular}{lcccc}
\hline$\beta_{1}$ & $\beta_{2}$ & Number of side-lobes & Main-lobe/Side-lobe intensity $(\mathrm{dB})$ & SNR $(\mathrm{dB})$ \\
\hline N.A. & N.A. & 0 & N.A. & 23.57 \\
$55^{\circ}$ & $55^{\circ}$ & 3 & 1.28 & 32.49 \\
$166^{\circ}$ & $110^{\circ}$ & 4 & 3.10 & 33.40 \\
$-139^{\circ}$ & $166^{\circ}$ & 4 & 6.92 & 35.31 \\
$55^{\circ}$ & $-139^{\circ}$ & 3 & 2.14 & 32.92 \\
$111^{\circ}$ & $166^{\circ}$ & 3 & 2.48 & 33.09 \\
\hline
\end{tabular}




\section{Chapter 6}

\section{Discussions and Conclusions}

We have presented the use of OBS to provide a LOS condition in an outdoor OWC link. Our study on shadowing in an OWC link illustrates the importance of the use of OBS in mitigating the effect of shadowing and reducing the BER. Conventional methods such as MEMS or SLM technology for implementing OBS exhibit low switching frequency which directly affects the steering speed. Therefore, it was essential to consider a solid-state OBS device which can function at much higher switching frequencies by implementing an OPA. Our study suggested the use of a doped silicon EOM OPA as opposed to $\mathrm{LiNbO}_{3}$ in order to provide a monolithic implementation.

Since previous work in OBS uses external low frequency signal processing for phase correction, we have proposed the use of an internal feedback which uses an optical PLL in order to correct for disturbances in the optical system. This provides the OBS device with much higher steering frequency. Furthermore, we have shown that this can be done by heterodyne down conversion or by amplitude sensing to correlate the phase correction required by the OPA in order to maintain coherent steering. Our work has demonstrated the steps to design a OBS to minimize internal cross-coupling using OptiBPM $^{\mathrm{TM}}$ and maximize directivity by simulating the radiation of a silicon OPA in free-space using MEEP $^{\mathrm{TM}}$.

Our results have shown that OBS increases the SNR in the desired direction. By individually shifting the phase of each waveguide element in the OPA, we were able to demonstrate OBS with $45^{\circ}$ steering range which is greater than previous monolithic 
OPAs. The simulation results illustrate an overall decrease of the HPBW and directivity as the waveguide spacing increases. We have shown that the directivity and HPBW is most sensitive to process variation of the waveguide spacing when a relative phase shift of $\beta_{1}$ and $\beta_{2}$ equals $166^{\circ}$ and $110^{\circ}$, respectively. The relative phase shifts which are least susceptible to process variation is when $\beta_{1}$ and $\beta_{2}$ are equal as well as when $\beta_{1}$ and $\beta_{2}$ equals $-139^{\circ}$ and $166^{\circ}$, respectively. Additionaly it has been demonstrated that the SNR can improve by 10-13 dB through the use of OBS. By simulating the effect in OPA dimension variation across varying phase shifts, the OWC system can be designed so as to minimize power consumption and achieve the desired HPBW, directivity, and steering range.

For maximum coupling, a coherent source with a fine linewidth such as a LD should be used for an OBS system. It was shown that the preferred use of source is a CW laser as opposed to a Gaussian beam which exhibits non-monotonic directivity. Future work considers accurate simulations of LEDs for coherent combining in free-space by implementing various optical filters. With regards to the power link budget, an improvement in resonator quality factor, LED output power, and coupling will be necessary for long distance OWC. 


\section{Appendices}

\section{Appendix A MEEP ${ }^{\mathrm{TM}}$ Source Code}

\section{A.1 CW source}

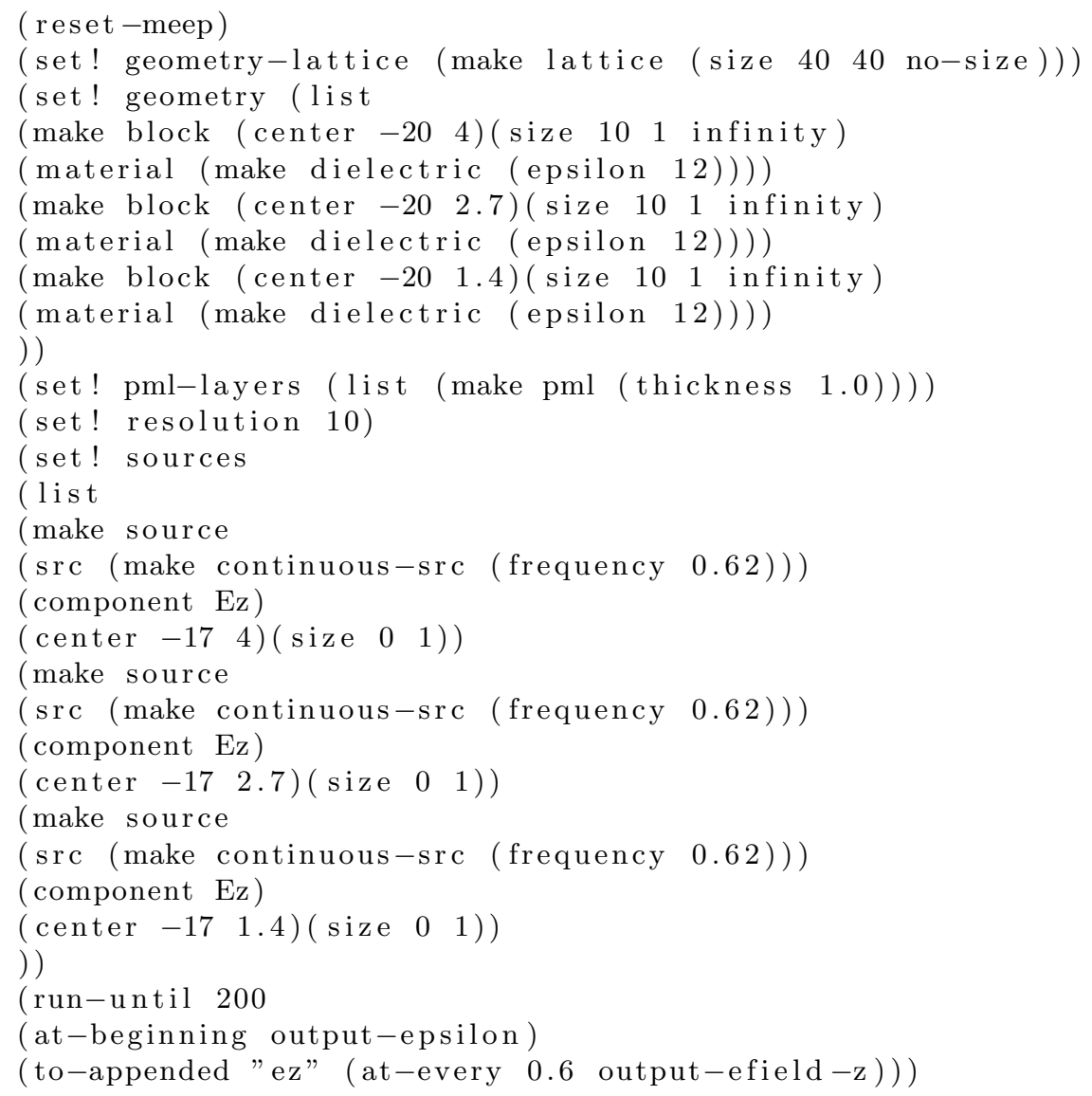

\section{A.2 Gaussian source}

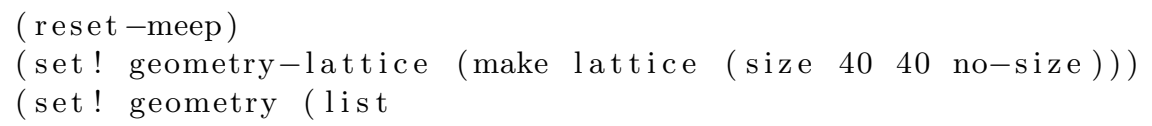




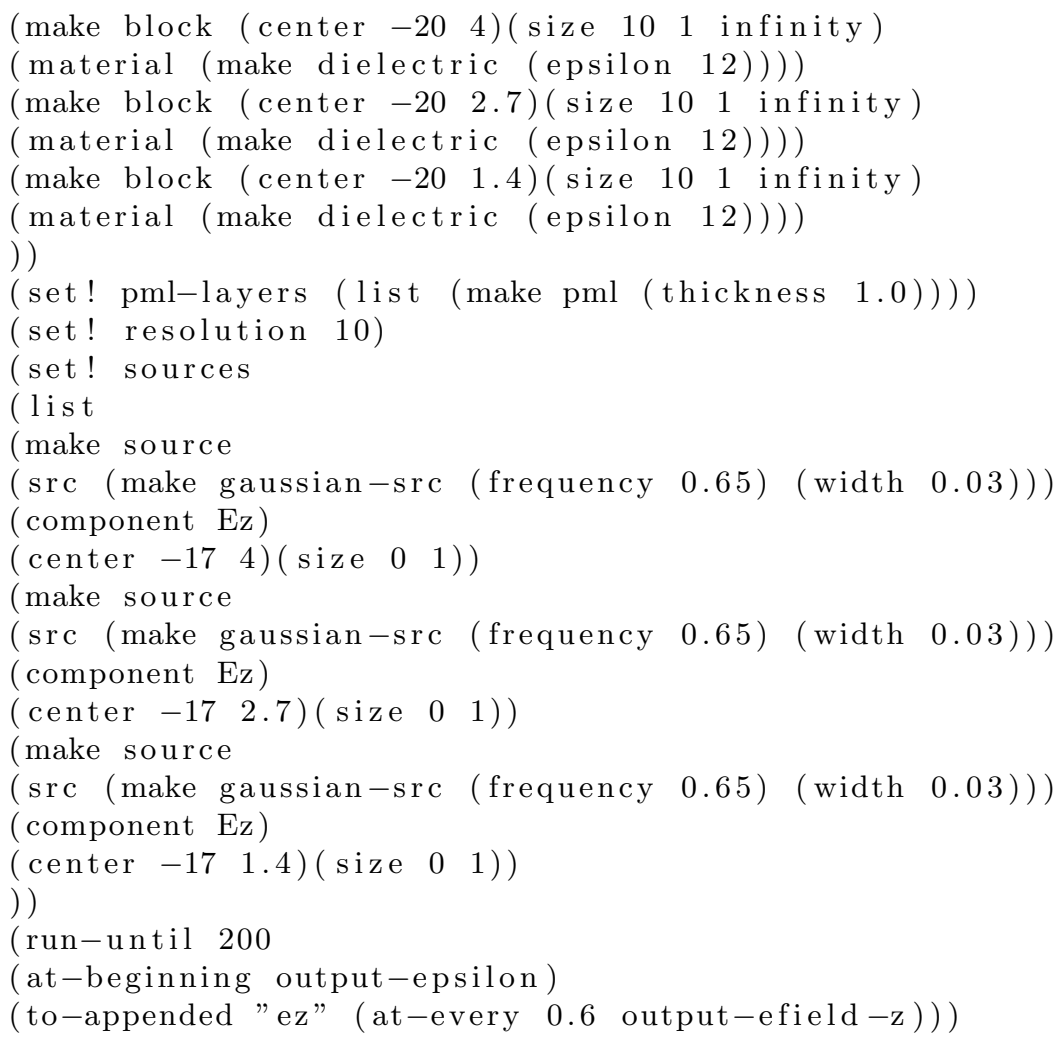

\section{A.3 Fiber implementation}

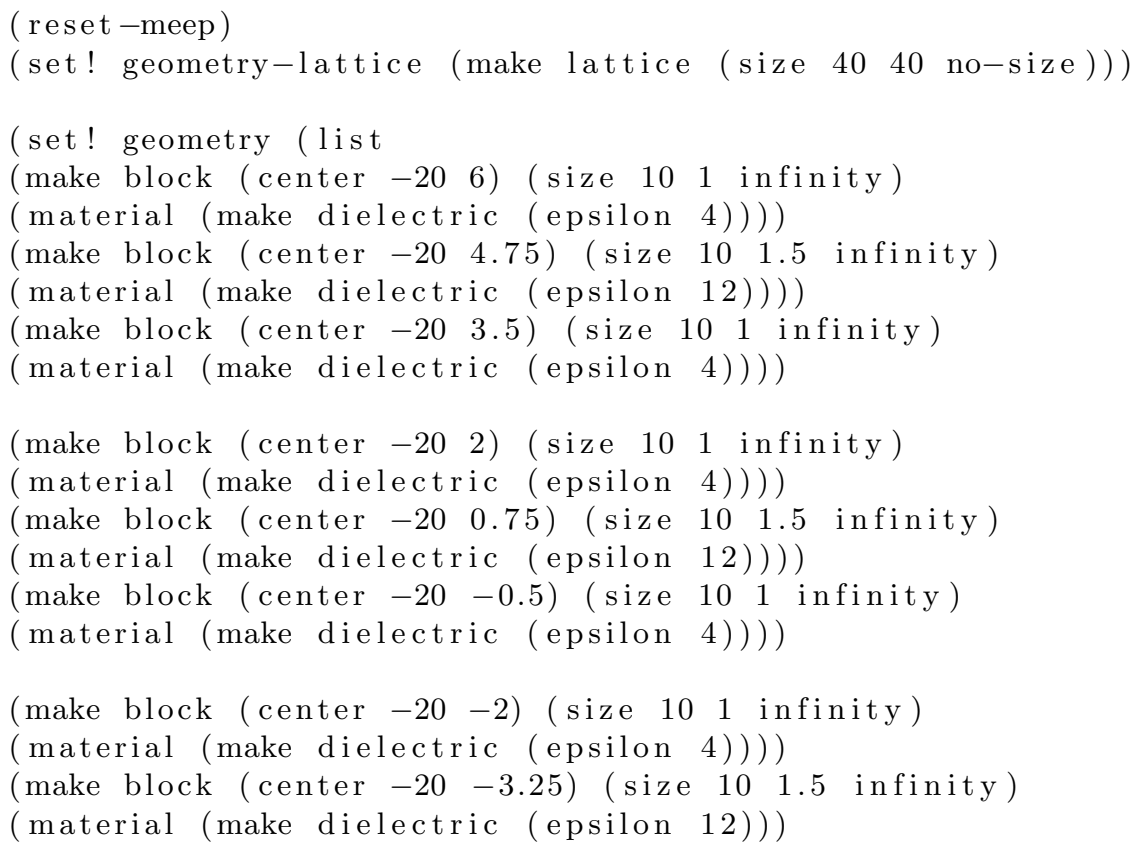




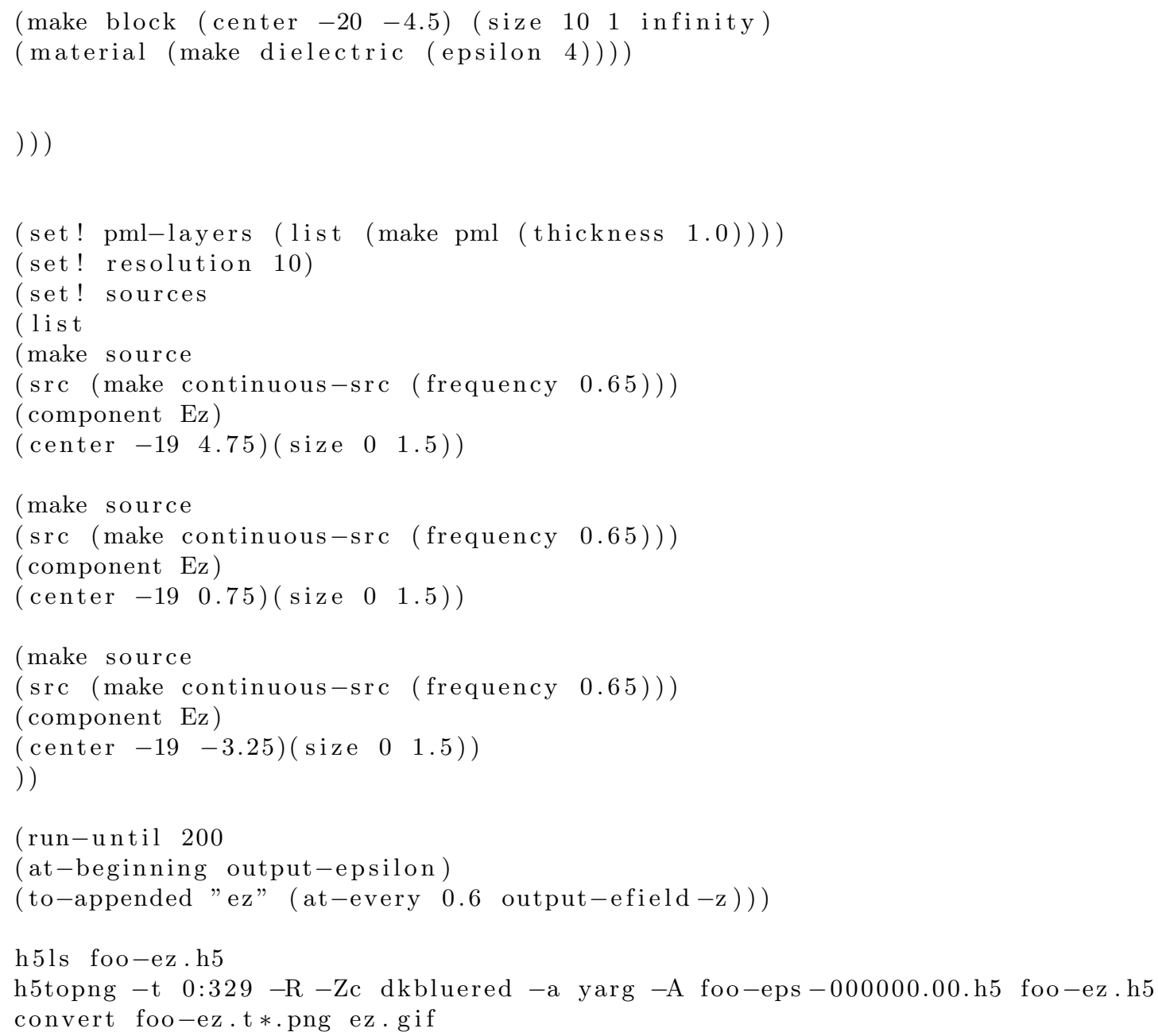

\section{Appendix B Python Source Code}

\section{B.1 Integrated SOI}

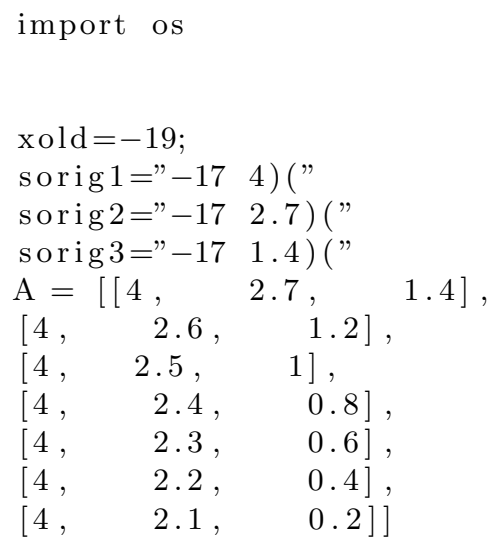




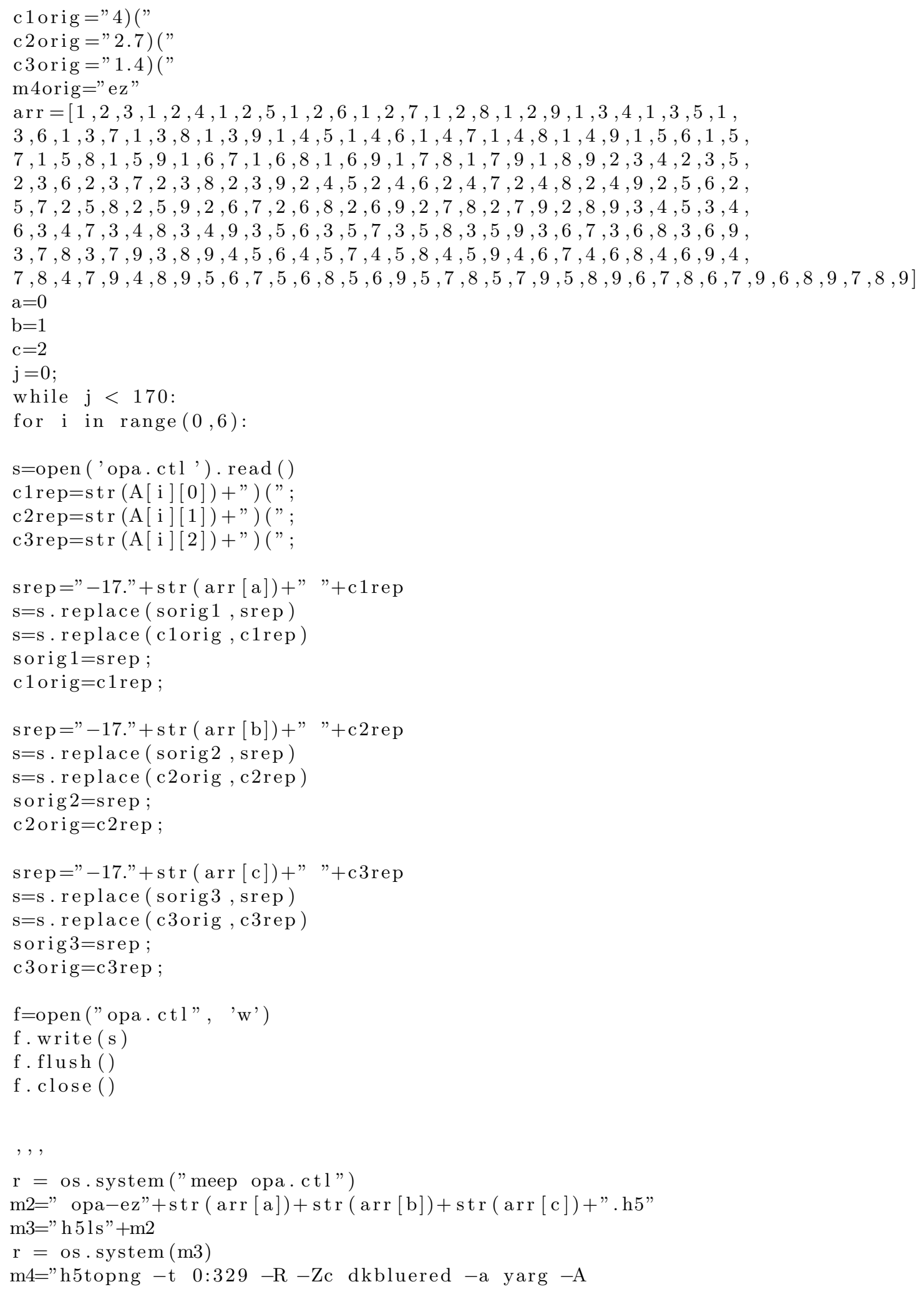




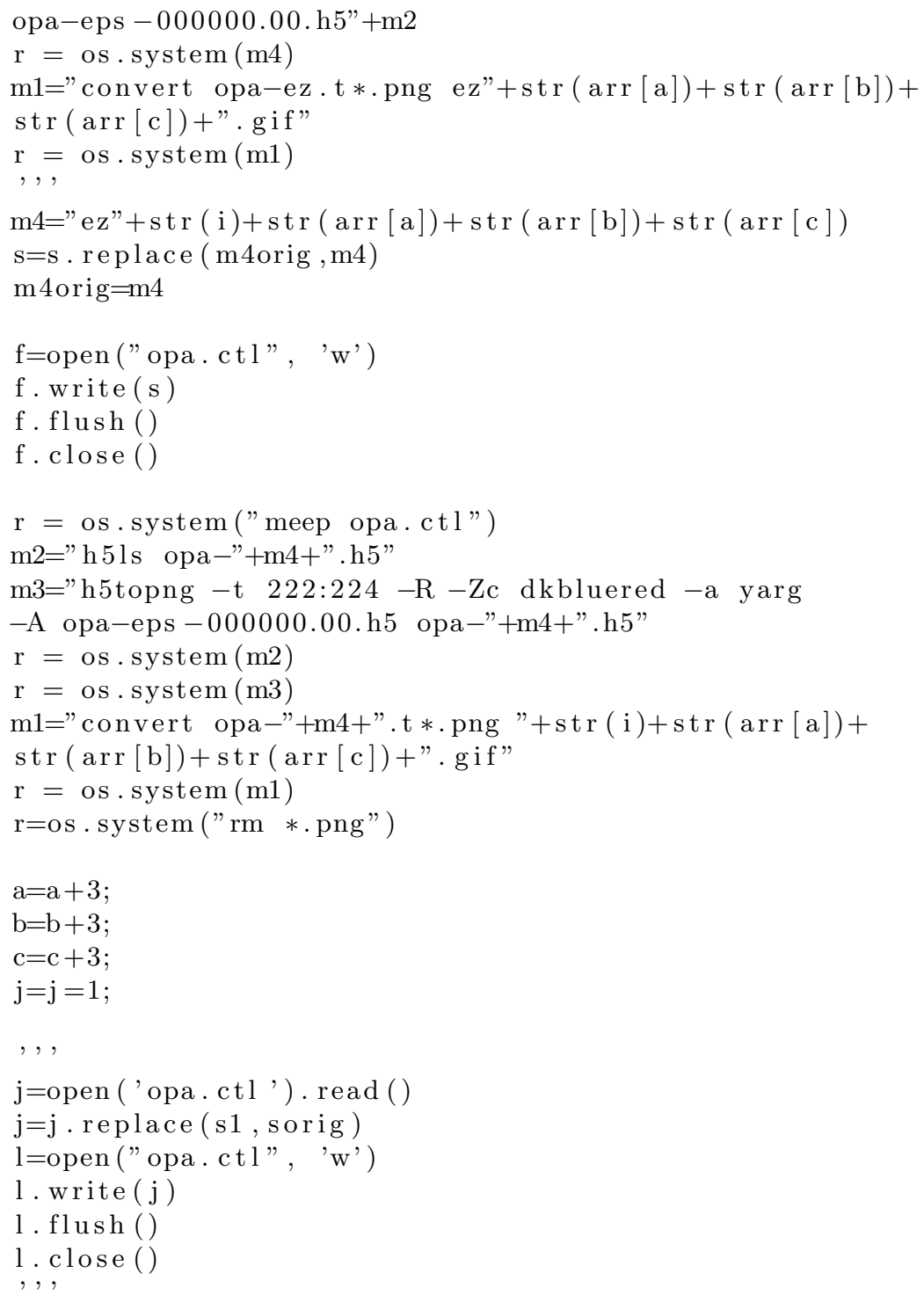

\section{B.2 Fiber implementation}

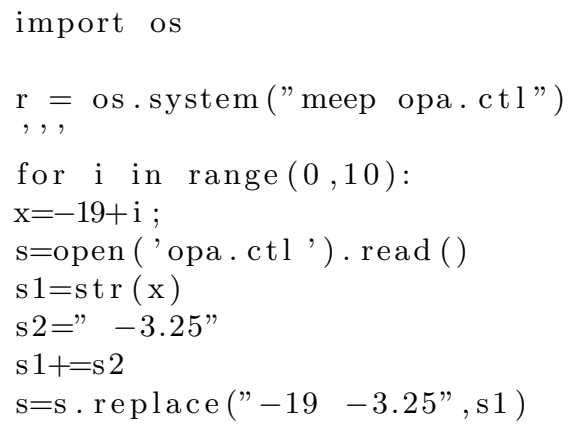




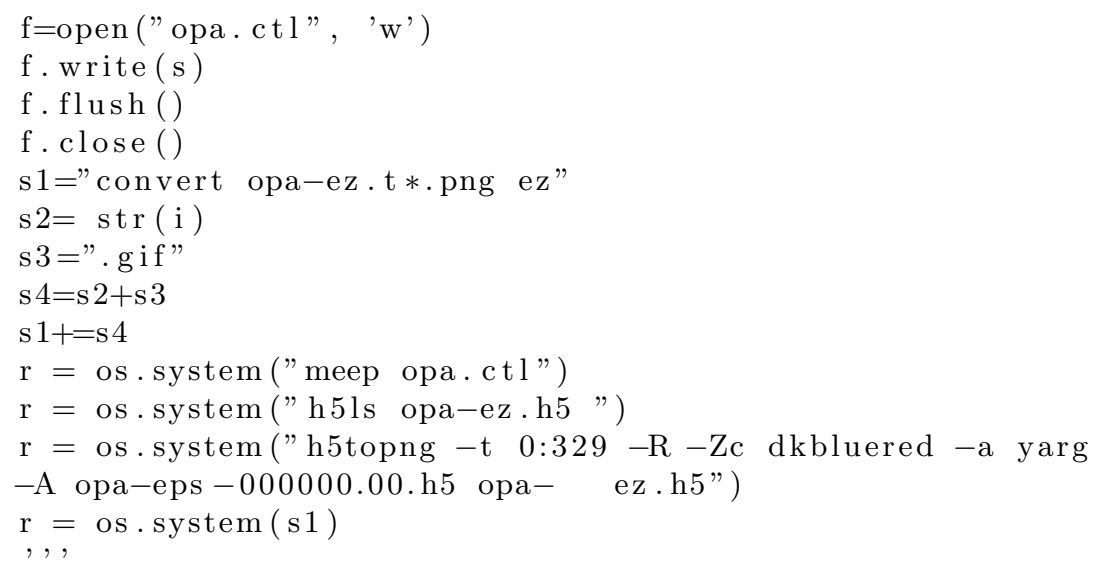

\section{B.3 SOI with coupling branches}

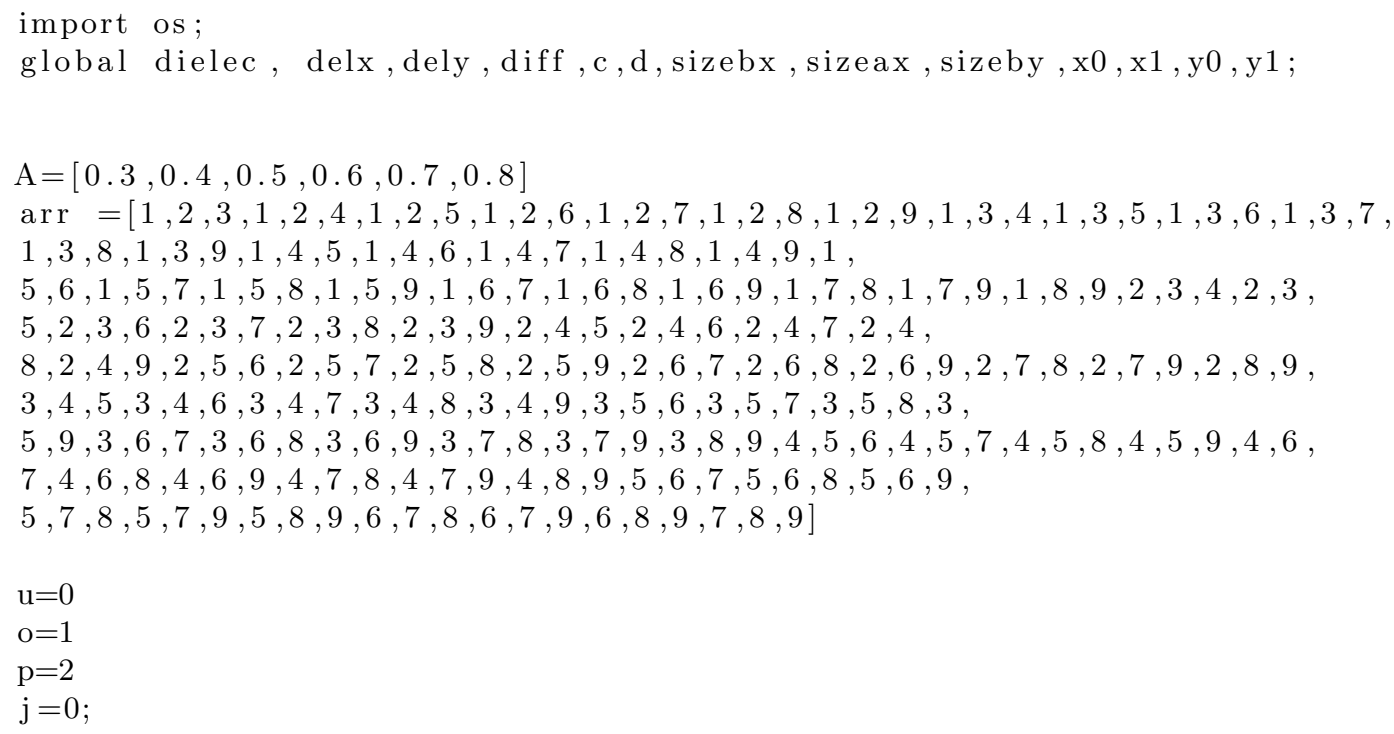




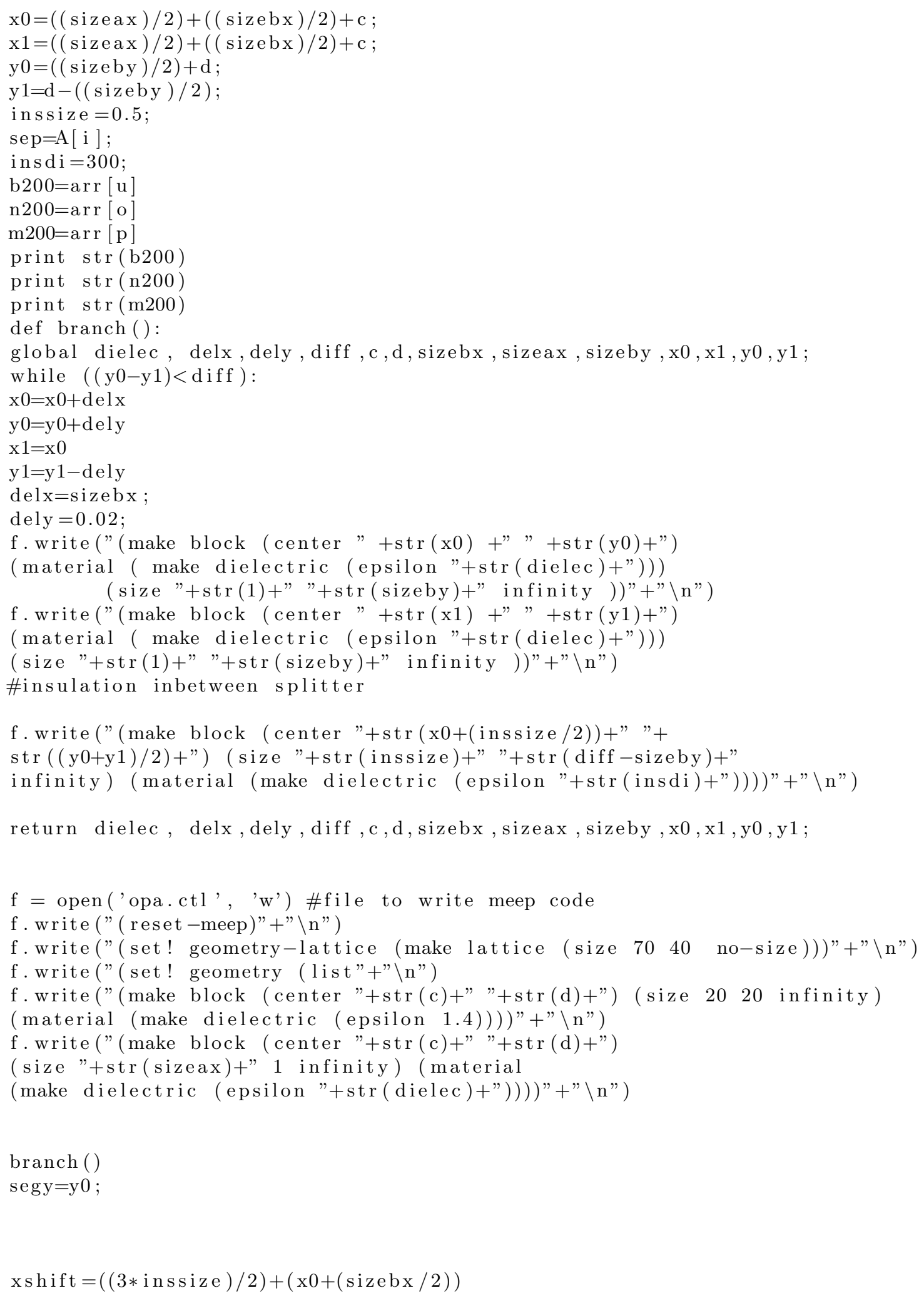




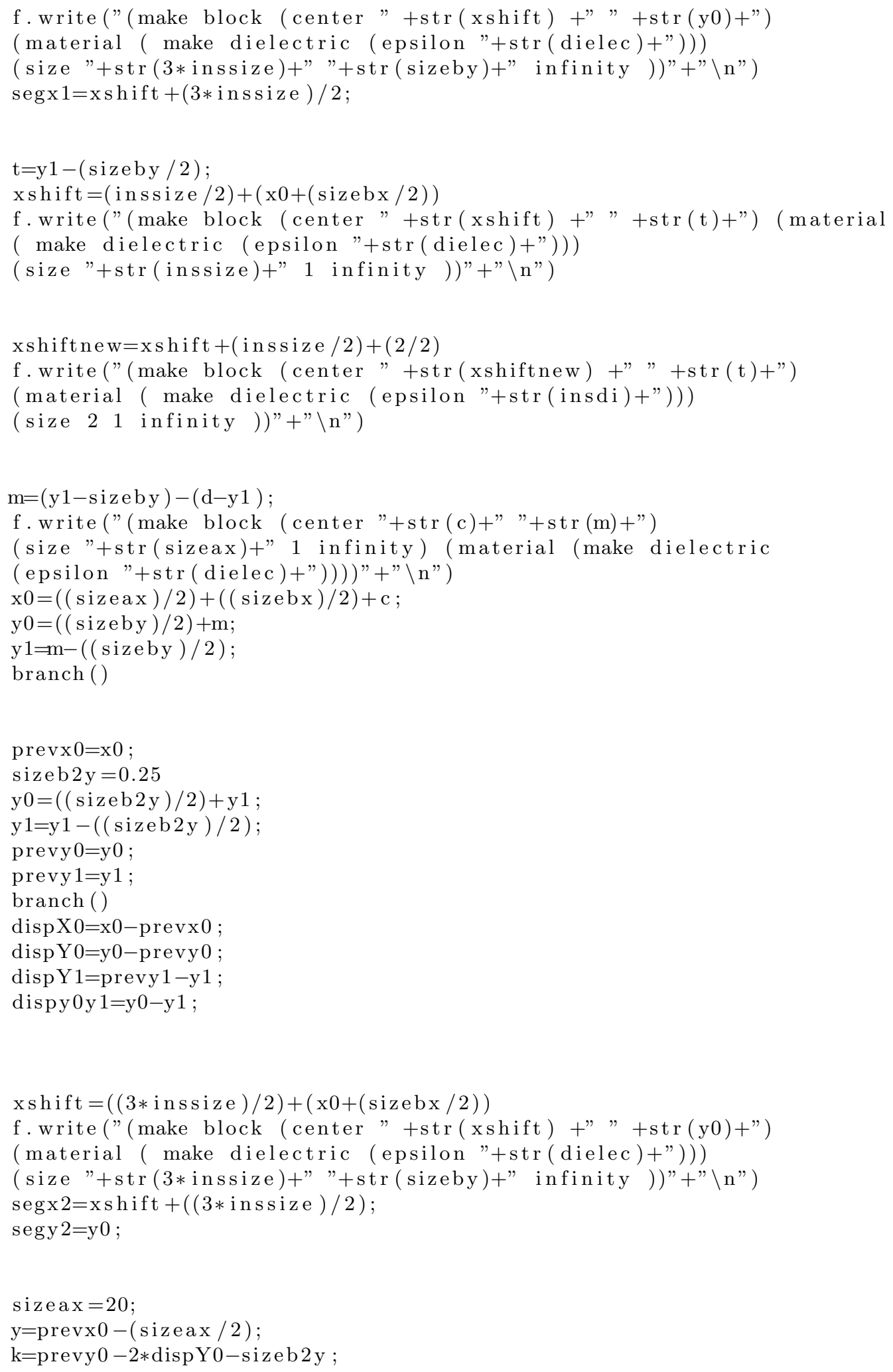




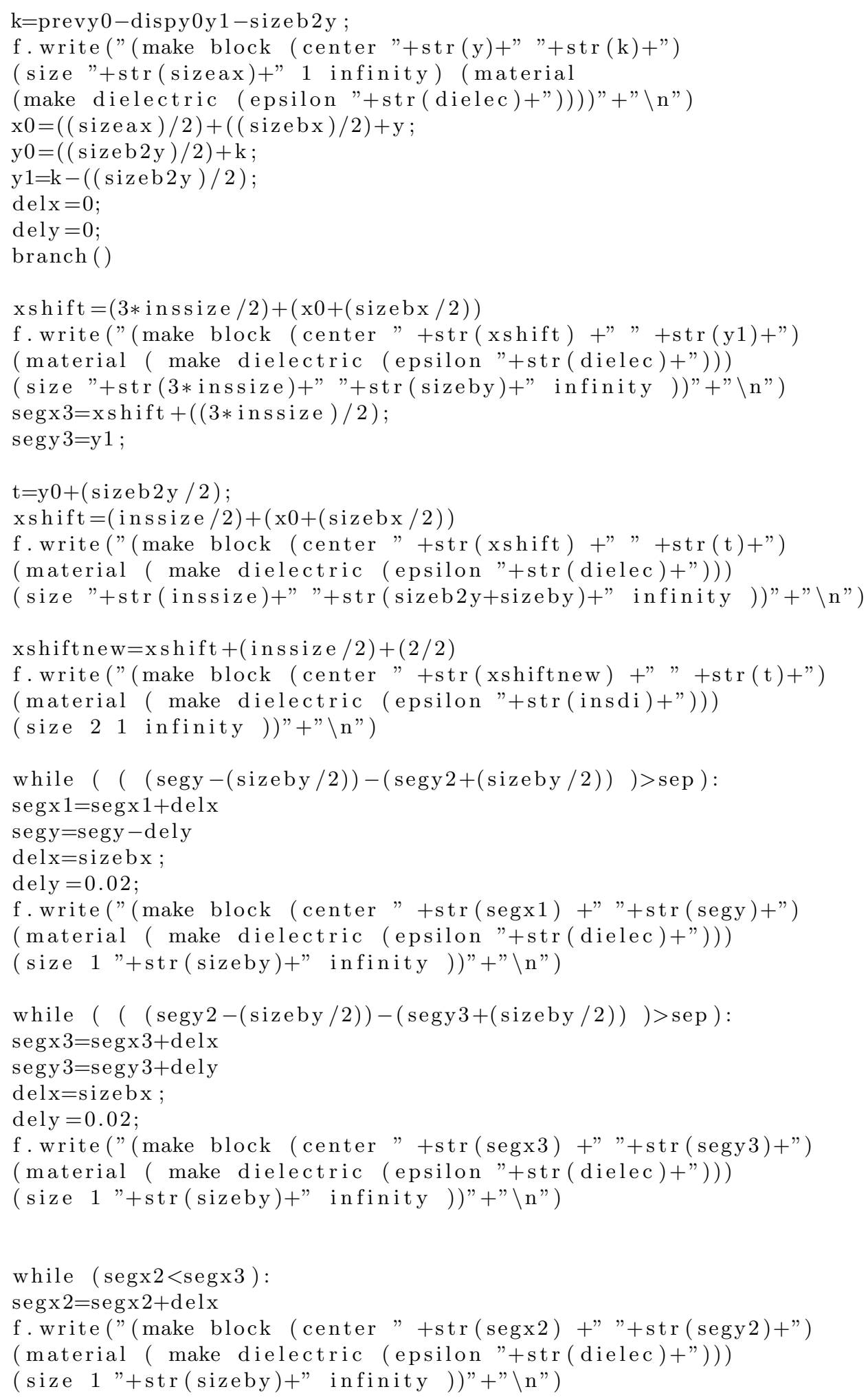




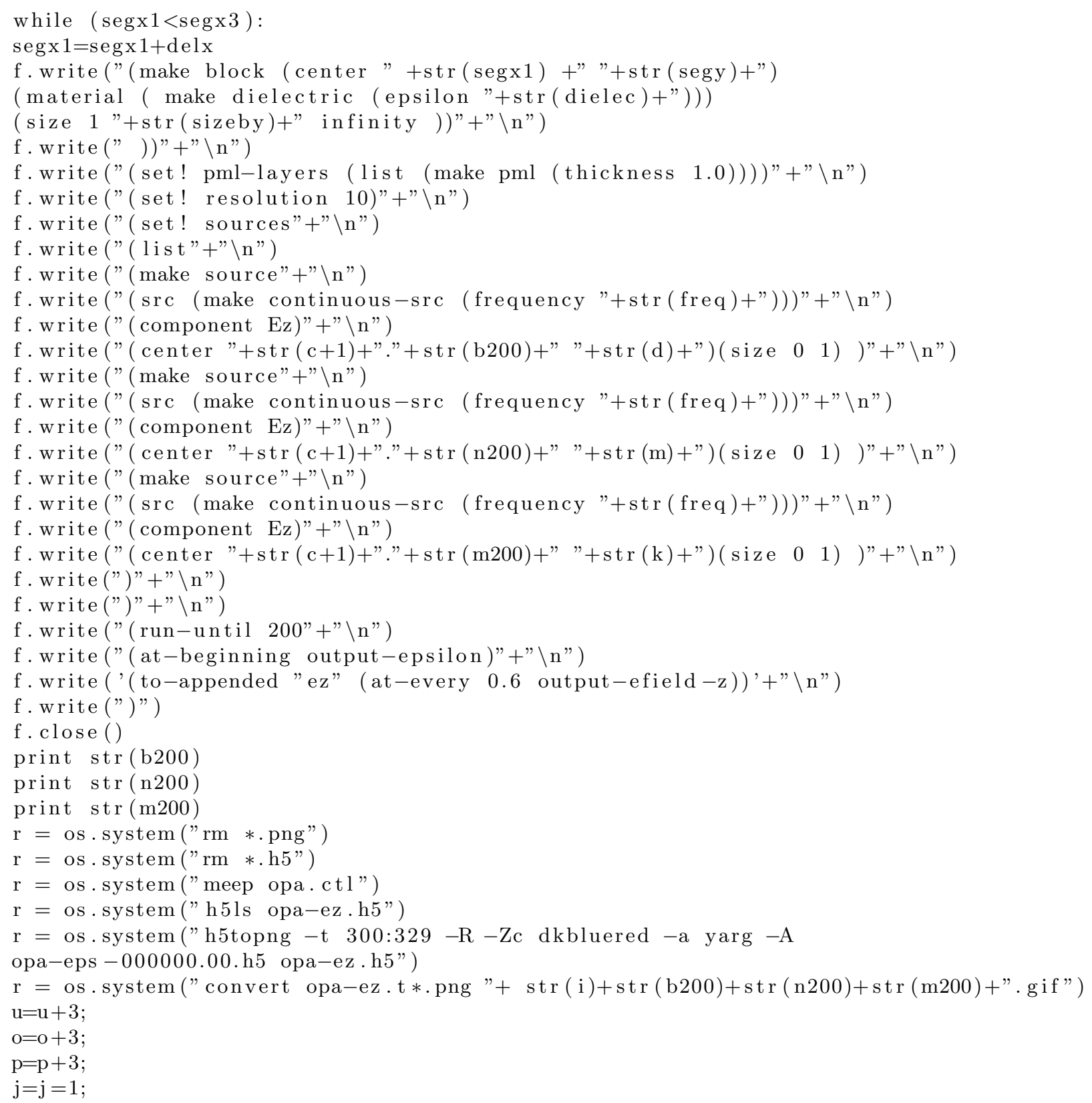




\section{Bibliography}

[1] R. Ramirez-Iniguez, S. M. Idrus, and Z. Sun, Optical wireless communications: IR for wireless Connectivity: V.9: Pts.1 \& 2, 2nd ed. Boca Raton: Auerbach Publishers, April 2008.

[2] P. F. McManamon, P. J. Bos, M. J. Escuti, J. Heikenfeld, S. Serati, H. Xie, and E. A. Watson, "A review of phased array steering for narrow-band electrooptical systems," Proceedings of the IEEE, vol. 97, no. 6, pp. 1078-1096, June 2009.

[3] W. R. Huang, J. Montoya, J. E. Kansky, S. M. Redmond, G. W. Turner, and A. Sanchez-Rubio, "High speed, high power one-dimensional beam steering from a 6-element optical phased array," Optics Express, vol. 20, no. 16, p. 17311, July 2012.

[4] J. K. Doylend, M. J. R. Heck, J. T. Bovington, J. D. Peters, L. A. Coldren, and J. E. Bowers, "Twodimensional free-space beam steering with an optical phased array on silicon-on-insulator," Optics Express, vol. 19, no. 22, p. 21595, October 2011.

[5] G. T. Reed and A. P. Knights, Silicon photonics: An introduction. Chichester, United Kingdom: John Wiley \& Sons, January 2004.

[6] Z. Ghassemlooy, W. Popoola, S. Rajbhandari, W. Popoola, and S. Rajbhandari, Optical wireless communications: System and channel Modelling with $M A T L A B(R)$. Boca Raton, FL: Taylor \& Francis, July 2012.

[7] P. Shams, M. Erol-Kantarci, and M. Uysal, "MAC layer performance of the IEEE 802.15.7 visible light communication standard," Transactions on Emerging Telecommunications Technologies, vol. 27, no. 5, pp. 662-674, January 2016.

[8] S. Dimitrov and H. Haas, Principles of LED light communications: Towards Networked Li-Fi. Cambridge, United Kingdom: Cambridge University Press, March 2015.

[9] A. Gomez, K. Shi, C. Quintana, M. Sato, G. Faulkner, B. C. Thomsen, and D. O'Brien, "Beyond 100-gb/s indoor wide field-of-view optical wireless communications," IEEE Photonics Technology Letters, vol. 27, no. 4, pp. 367-370, February 2015.

[10] A. Khalifeh, H. Farahneh, C. Mekhiel, and X. Fernando, "Visible light communication numerous applications," in Encyclopedia of Information Science and Technology, 4th ed. IGI Global, 2016.

[11] A. F. Oskooi, D. Roundy, M. Ibanescu, P. Bermel, J. Joannopoulos, and S. G. Johnson, "Meep: A flexible free-software package for electromagnetic simulations by the FDTD method," Computer Physics Communications, vol. 181, no. 3, pp. 687-702, March 2010.

[12] N. Blaunstein, S. Arnon, and N. Kopeika, Applied aspects of optical communication and LIDAR. Boca Raton: CRC Press, December 2009.

[13] H. Nikkhah, K. V. Acoleyen, and R. Baets, "Beam steering for wireless optical links based on an optical phased array in silicon," annals of telecommunications - annales des télécommunications, vol. 68, no. 1-2, pp. 57-62, June 2012.

[14] G. Stamenovi, S. R. Pani, D. Rani, aslav Stefanovi, and M. Stefanovi, "Performance analysis of wireless communication system in general fading environment subjected to shadowing and interference," EURASIP Journal on Wireless Communications and Networking, vol. 2014, no. 1, p. 124, 2014. 
[15] H. Farahneh, C. Mekhiel, A. Khalifeh, W. Farjow, and X. Fernando, "Shadowing effects on visible light communication channels," in IEEE Canadian Conference on Electrical and Computer Engineering (CCECE) - Communications and Networks, Vancouver, BC, Canada, May 2016.

[16] S. Hranilovic, Wireless optical communication systems. New York: Springer-Verlag New York, September 2004.

[17] R. S. Berns and D. M. Reiman, "Color managing the third edition of bill meyer and saltzman's principles of color technology," Color Research \& Application, vol. 27, no. 5, pp. 360-373, August 2002.

[18] T. Komine, S. Haruyama, and M. Nakagawa, "A study of shadowing on indoor visible-light wireless communication utilizing plural white LED lightings," Wireless Personal Communications, vol. 34, no. 1-2, pp. 211-225, July 2005.

[19] F. Gfeller and U. Bapst, "Wireless in-house data communication via diffuse infrared radiation, rz 941 (32513)," Information Systems, vol. 5, no. 3, p. 248, January 1980.

[20] L. Zeng, D. O’Brien, H. Minh, G. Faulkner, K. Lee, D. Jung, Y. Oh, and E. Won, "High data rate multiple input multiple output (MIMO) optical wireless communications using white LED lighting," IEEE Journal on Selected Areas in Communications, vol. 27, no. 9, pp. 1654-1662, December 2009.

[21] A. Bazzi, B. M. Masini, A. Zanella, and G. Pasolini, "IEEE 802.11p for cellular offloading in vehicular sensor networks," Computer Communications, vol. 60, pp. 97-108, April 2015.

[22] C. Zhang, B. E. Mapes, and B. J. Soden, "Bimodality in tropical water vapour," Quarterly Journal of the Royal Meteorological Society, vol. 129, no. 594, pp. 2847-2866, October 2003.

[23] C. Forbes, M. Evans, N. Hastings, and B. J. Peacock, Statistical distributions, 4th ed. United States: Wiley, John \& Sons, January 2011.

[24] J. Carruthers and J. Kahn, "Modeling of nondirected wireless infrared channels," IEEE Transactions on Communications, vol. 45, no. 10, pp. 1260-1268, 1997.

[25] K.-P. Ho, Phase-modulated optical communication systems. New York: Springer Science+Business Media, July 2005.

[26] C. Mekhiel and X. Fernando, "LED beam steering for Li-Fi communications," in IEEE International Workshop on Computer Aided Modelling and Design of Communication Links and Networks (CAMAD), Toronto, ON, Canada, October 2016.

[27] D. Christodoulides, L. Reith, and M. Saifi, "Theory of LED coupling to single-mode fibers," Journal of Lightwave Technology, vol. 5, no. 11, pp. 1623-1629, 1987.

[28] P. E. P. H. S. J. M. I. S. M. Kaarre, S. Kivi and M. Juuti, "Development of tunable fabry-perot spectral camera and light source for medical applications," in 2013 AIP Conference Proceedings, Saint Petersburg, Russia, May 2012.

[29] C. Wang and J. Yao, "Fiber bragg gratings for microwave photonics subsystems," Optics Express, vol. 21, no. 19, p. 22868, September 2013.

[30] W. Bogaerts, P. D. Heyn, T. V. Vaerenbergh, K. D. Vos, S. K. Selvaraja, T. Claes, P. Dumon, P. Bienstman, D. V. Thourhout, and R. Baets, "Silicon microring resonators," Laser E Photonics Reviews, vol. 6, no. 1, pp. 47-73, September 2011.

[31] D. Rabus, "Ring resonators: Theory and modeling," in Integrated Ring Resonators: The Compendium. Berlin/Heidelberg,DE: Spring, 2007, pp. 3-40.

[32] H.-W. Chen, Y. hao Kuo, and J. E. Bowers, "High speed hybrid silicon evanescent mach-zehnder modulator and switch," Optics Express, vol. 16, no. 25, p. 20571, November 2008.

[33] G. Keiser, "Power launching and coupling," in Optical Fiber Communications, 2nd ed. Singapore: McGrawHill Series in Electrical Engineering, 2003, pp. 196-226.

[34] C. A. Balanis, Antenna theory: Analysis and design, 3rd ed. Chichester, United Kingdom: Wiley-Blackwell (an imprint of John Wiley \& Sons Ltd), July 2005.

[35] C. Mekhiel and X. Fernando, "Free-space optical beam steering feasibility for wireless communications," 2016 Photonics north (PN), May 2016. 
[36] M. Toyoshima, "Maximum fiber coupling efficiency and optimum beam size in the presence of random angular jitter for free-space laser systems and their applications," Journal of the Optical Society of America $A$, vol. 23, no. 9, p. 2246, September 2006. 